\title{
DIVERGENT PLUME REDUCTION OF A HIGH-EFFICIENCY MULTISTAGE PLASMA THRUSTER
}

\author{
A Thesis \\ presented to \\ the Faculty of California Polytechnic State University, \\ San Luis Obispo
}

In Partial Fulfillment

of the Requirements for the Degree

Master of Science in Aerospace Engineering

by

Christopher M. Barlog

January 2016 
(C) 2016

Christopher M. Barlog

ALL RIGHTS RESERVED 


\section{COMMITTEE MEMBERSHIP}

TITLE:

Divergent Plume Reduction of a High-Efficiency

Multistage Plasma Thruster

AUTHOR:

Christopher M. Barlog

DATE SUBMITTED:

January 2016

COMMITTEE CHAIR: $\quad$ Kira Abercromby, Ph.D.

Assistant Professor of Aerospace Engineering

COMMITTEE MEMBER: Dan Goebel, Ph.D.

Senior Research Scientist at Jet Propulsion Laboratory

COMMITTEE MEMBER: Raymond Liang, Ph.D.

Senior R\&D Engineer at Space Systems Loral

COMMITTEE MEMBER: $\quad$ Eric Mehiel, Ph.D.

Professor of Aerospace Engineering 


\begin{abstract}
Divergent Plume Reduction of a High-Efficiency Multistage Plasma Thruster
\end{abstract}

\title{
Christopher M. Barlog
}

High Efficiency Multistage Plasma Thrusters (HEMPTs) are a relatively new form of electric propulsion that show promise for use on a variety of missions and have several advantages over their older EP competitors. One such advantage is their long predicted lifetime and minimal wall erosion due to a unique periodic permanent magnet system. A laboratory HEMPT was built and donated by JPL for testing at Cal Poly. Previous work was done to characterize the performance of this thruster and it was found to exhibit a large plume divergence, resulting in decreased thrust and specific impulse. This thesis explores the design and application of a magnetic shield to modify the thruster's magnetic field to force more ion current towards the centerline. A previous Cal Poly thesis explored the same concept, and that work is continued and furthered here. The previous thesis tested a shield which increased centerline current but decreased performance. A new shield design which should avoid this performance decrease is studied here.

Magnetic modelling of the thruster was performed using COMSOL. This model was verified using guassmeters to measure the field strength at many discrete points within and near the HEMPT, with a focus on the ionization channel and exit plane. A shield design which should significantly reduce the radial field strength at the exit plane without affecting the ionization channel field was modelled and implemented. The HEMPT was tested in a vacuum chamber with and without the shield to characterize any change to performance characteristics. Data were collected using a nude Faraday probe and retarding potential analyzer. The data show a significant increase in centerline current with the application of the shield, but due to RPA malfunction and thruster failure the actual change in performance could not be concluded. 
The unshielded HEMPT was characterized, however, and was found to produce 12.1 $\pm 1.3 \mathrm{mN}$ of thrust with a specific impulse of $1361 \pm 147 \mathrm{~s}$. The thruster operated with a total efficiency of $10.63 \pm 3.66 \%$, an efficiency much lower than expected. A large contributor to this low efficiency is likely the use of argon in place of xenon. Its lower mass and higher ionization energy make it a less efficient propellant choice. Further, the thruster is prone to overheating, indicating that significant thermal losses are present in this design. 


\section{ACKNOWLEDGMENTS}

I would like to recognize here all of the people who made this thesis possible, and there is no one who has been more instrumental in its completion than Dr. Dan Goebel of JPL. Dan is a seemingly endless source of knowledge on all topics relating to EP, from theory to testing. I had no experience and only a very basic understanding of electric propulsion concepts at the outset of this project. Dan was always willing to share his vast experience, whether it was through detailed and quick email exchanges or facility visits to teach me about EP measurement and diagnose problems with my experimental setup. He was available for any question I had, no matter how small. He also generously loaned me necessary materials and equipment, without which this thesis would not have been possible. I am extremely grateful for all of his help.

I would also like to extend a huge thank you to my thesis advisor, Dr. Kira Abercromby. She is responsible for first sparking my interest in plasma physics and EP during my junior year in her Space Environments course. Her joyful and open-minded approach to problems was always refreshing. She was available for any and all problems or questions that came up throughout this thesis (and there were plenty). Her knowledge of vacuum chambers and other equipment in the lab was also invaluable. Even though propulsion isn't her area of expertise, and she was managing what probably felt like 30 other grad students, she always showed a genuine interest in this project and my progress.

A big thanks to Dr. Raymond Liang for his assistance with this undertaking as well. Firstly, I want to thank him for agreeing to be on my thesis committee on such short notice. His expertise in EP testing proved a very useful resource. He was always available to answer any questions I had with setting up the experiment, an endeavor with which I had very little experience. His wisdom saved me a lot of time. He also provided useful insight during the data analysis phase. I also want to give him credit for keeping me on track. When I hadn't provided him with an update in a while he would reach out to me and check in, a small action which, more than once, gave me motivation to put my other schoolwork aside and work more on this project. 
I would also like to thank Dr. Eric Mehiel for joining my committee and questioning some of the early choices I was making regarding the magnetic field model. I want to thank Dr. Rob McDonald, as well, for providing helpful feedback during my thesis seminar.

I owe an enormous thanks to both Scott McGrail and Max Bodnar. To Scott, because he laid so much of the groundwork for this project, including putting in many hours getting the chamber to a point where it could reach the desired pressure, collecting the required equipment, and providing me access to much of his research, images, and data. His thesis served as a partial roadmap and guidebook in the completion of my own. It was Scott who was the initiate of this project, and being first to implement such a complicated test setup I'm sure was no easy task. He was also very available and willing to offer insight from his experience whenever I asked. My thesis is built on the strong foundation that he laid in his, and I'm grateful for that.

To Max Bodnar, because he was present in the lab working on his thesis while I was working on the early stages of my own. This was when I was in need of the most guidance, and so often he would be the first person I would turn to. In most cases, he was both the first and the last person because his advice was very thorough. I also leaned on him for many questions about hollow cathode operation when I felt like Dan could use a break. Even when I accidentally messaged him while he was enjoying travel abroad he took the time to write up a detailed response to my question.

I would like to acknowledge Cody Thompson for his help with almost all of the welding and machining necessary for this project. He has a depth of experience with manufacturing that proved extremely useful for this project. Also, when the chamber's hoist broke he was very accessible to help me remove the cover using the forklift. Further, I'd like to thank Dr. Richard Savage and the Biomedical Engineering department for their generosity in allowing me to use the department's helium leak detector, a tool which I credit with saving my sanity when I was trying to integrate the propellant feed system and get the chamber to reach an acceptable pressure. 
A big thanks to Tim Fitzgerald and my sister, Emily, for taking time out of their days to hang out in the hot lab in the midst of summer to make sure I didn't electrocute myself during testing.

I want to thank my siblings and friends for their love and support throughout this. And, last but far from least, I am extremely thankful for my wonderful parents, Michael and Susan Barlog. From before I could even talk to the present day, they have always put an emphasis on learning and provided me with access to a great education. I would never have considered pursuing a master's degree or be where I am today if not for their encouragement, past and present. 


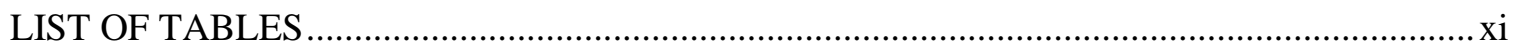

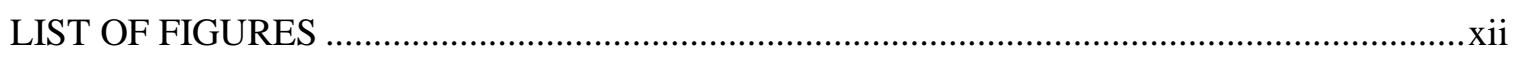

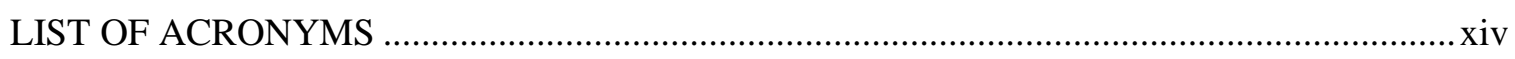

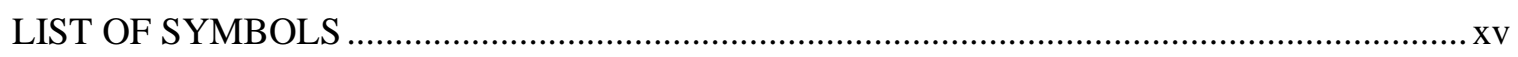

CHAPTER

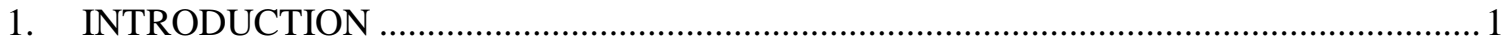

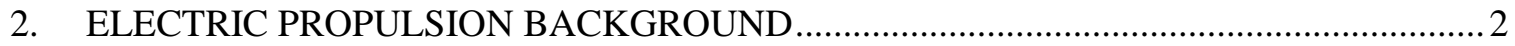

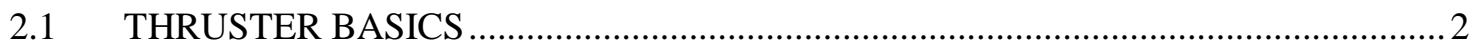

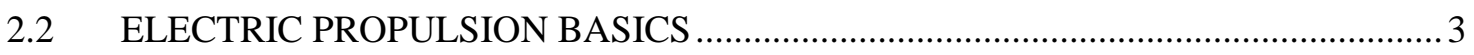

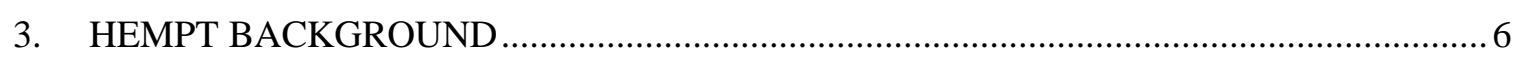

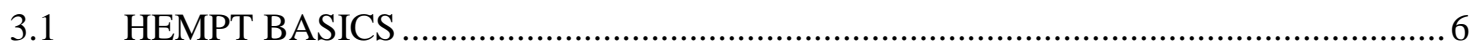

3.2 HEMPT DIMENSIONS AND HOLLOW CATHODE …......................................... 8

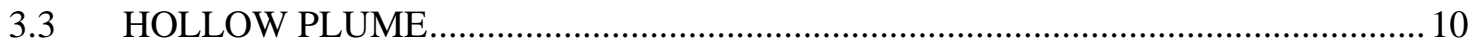

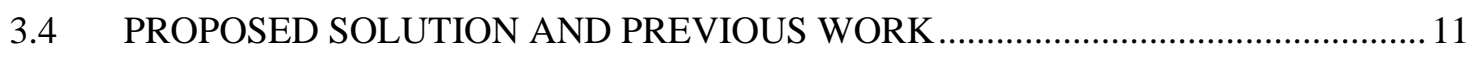

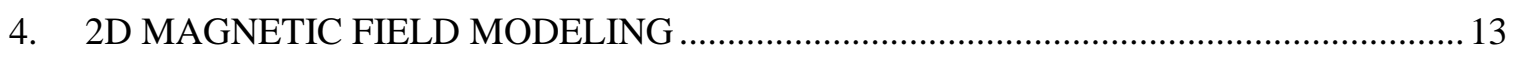

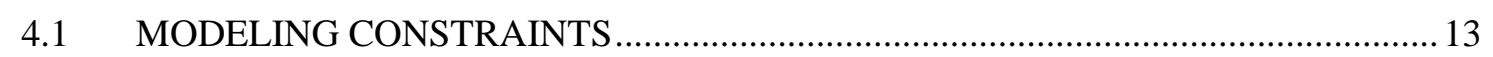

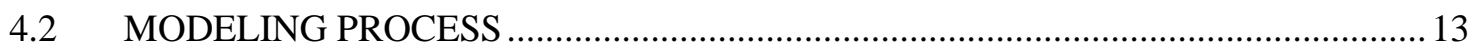

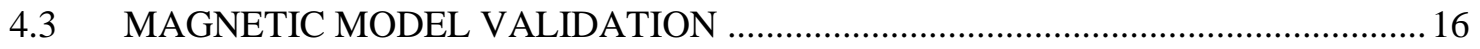

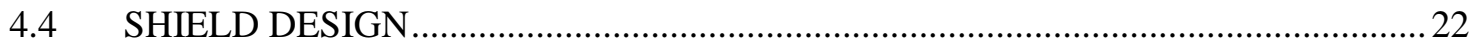

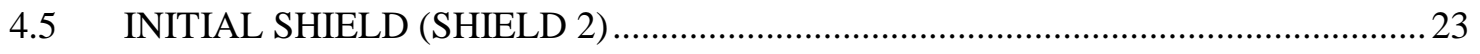

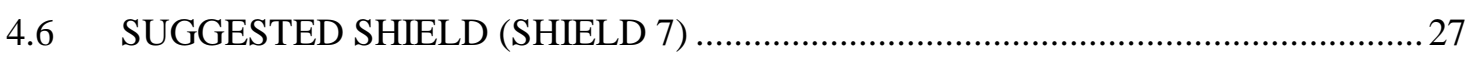

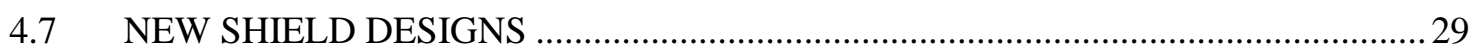

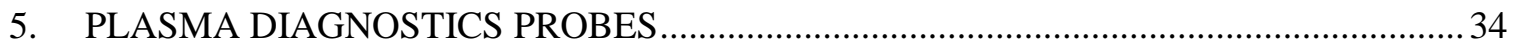

5.1 RETARDING POTENTIAL ANALYZER (RPA) .................................................. 34

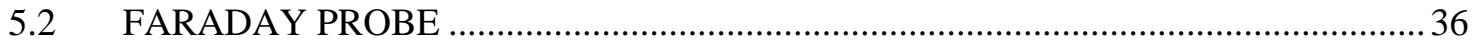

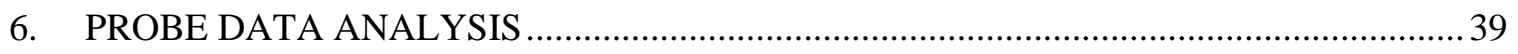

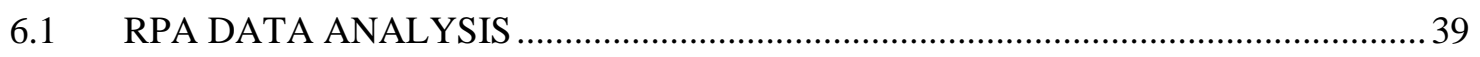

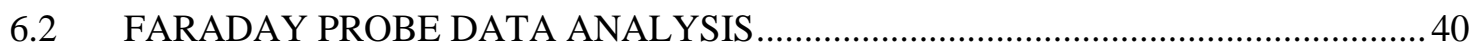

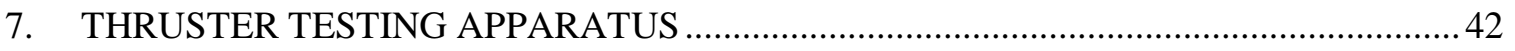

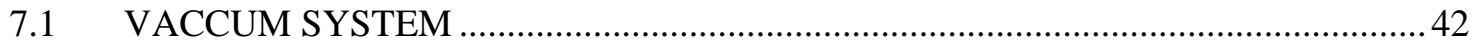

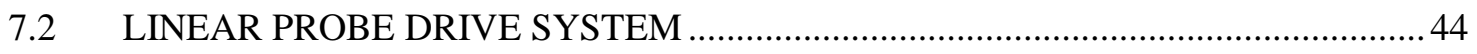

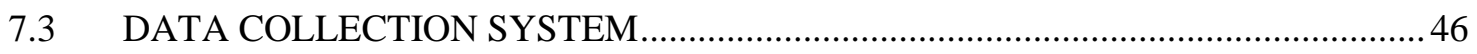

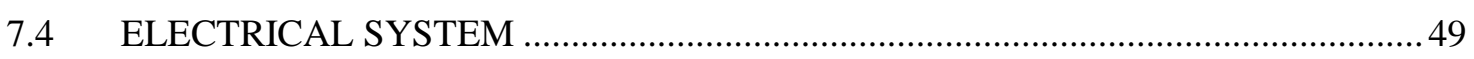

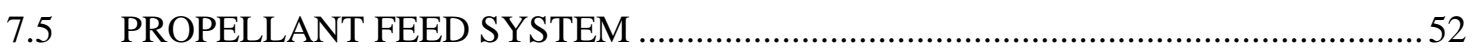




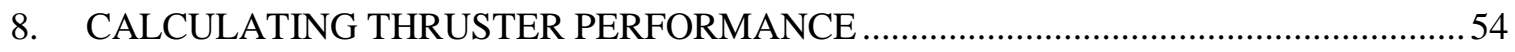

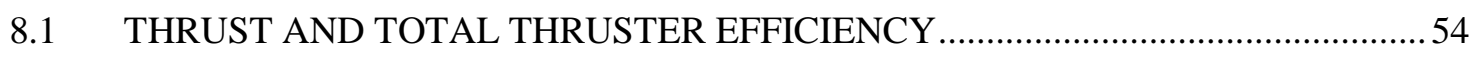

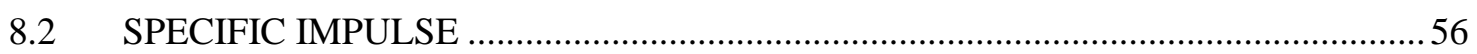

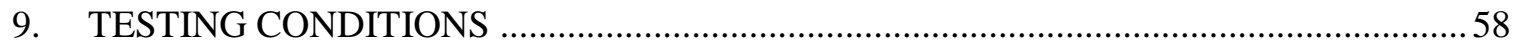

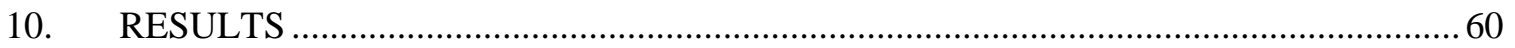

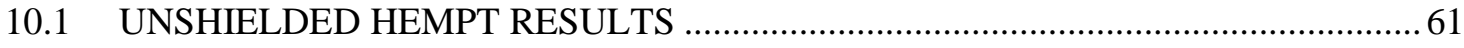

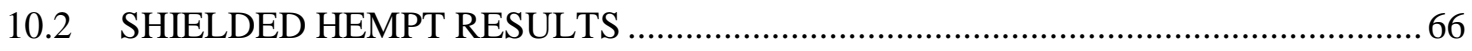

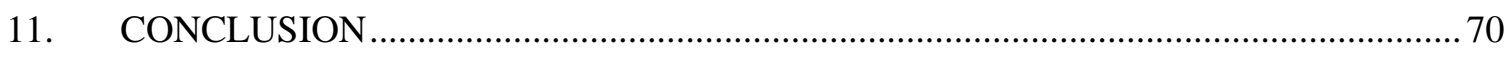

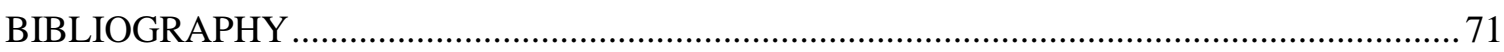

APPENDICES

A. LESSONS LEARNED AND RECOMMENDATIONS ............................................. 73

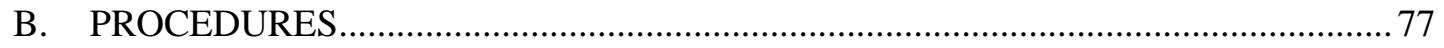

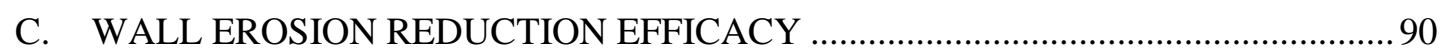

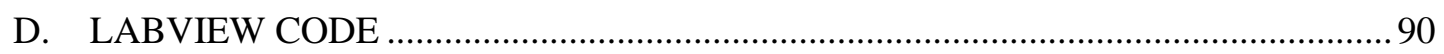




\section{LIST OF TABLES}

Table

Page

1. Material Properties Used in HEMPT Physics Model ........................................................... 13

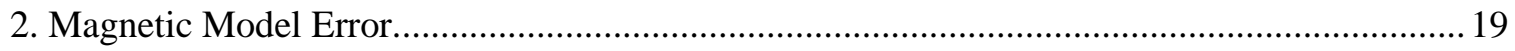

3. Radial Magnetic Field Strength, $\mathrm{B}_{\mathrm{r}}$, at X-Locations at Ionization Chamber Wall.................... 26

4. Summary of Modeled Magnetic Shield Performance............................................................ 32

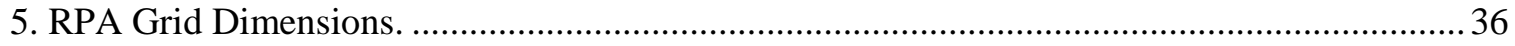

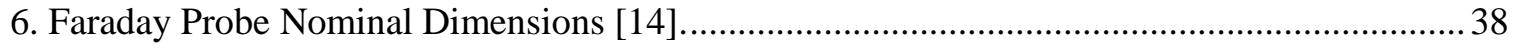

7. Resistance and Capacitance Values for Data Collection. .......................................................... 48

8. List of Power Supplies Used for HEMPT Testing................................................................50

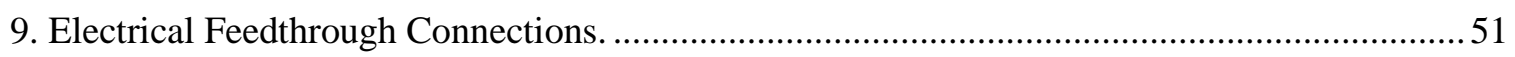

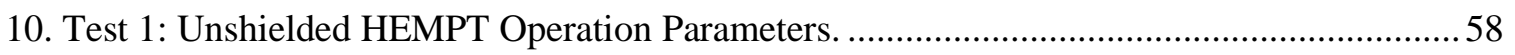

11. Test 2: Shielded HEMPT Operation Parameters...................................................................59

12. Test 3: Unshielded HEMPT Operation Parameters. …..........................................................59

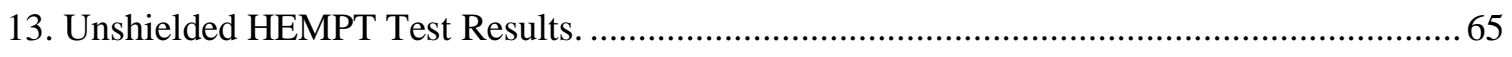

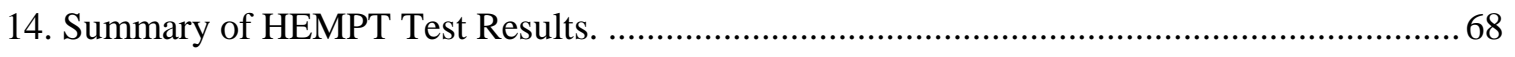




\section{LIST OF FIGURES}

Figure $\quad$ Page

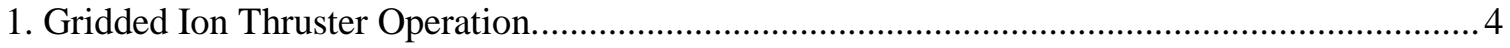

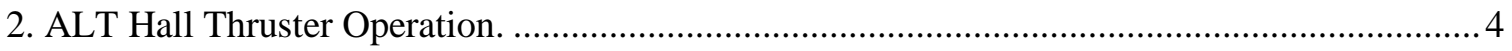

3. High Efficiency Multistage Plasma Thruster Operation....................................................... 7

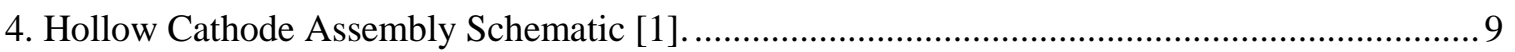

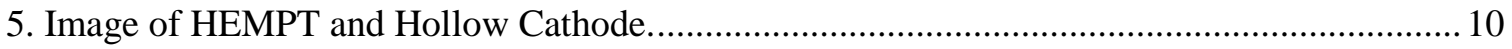

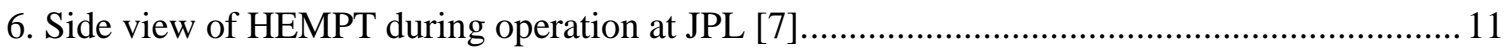

7. Surface Plot of the Norm of Magnetic Flux Density, $|\mathrm{B}|$, with Overlaid Streamlines of

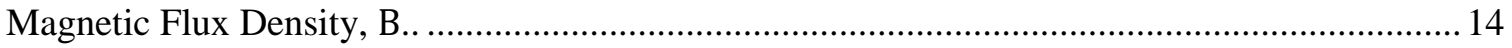

8. HEMPT Magnetic Model Produced in MagNet by Scott McGrail [15].................................. 15

9. COMSOL HEMPT Magnetic Model Overlaid on MagNet Model .......................................... 15

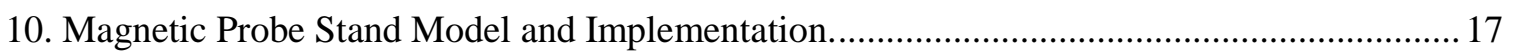

11. Empirical and Theoretical Arrow Surface Plot of HEMPT Magnetic Field............................ 18

12. Histogram of Angle Error Between Measured and Predicted Field. ....................................... 20

13. Histogram of Magnitude Error between Measured and Predicted Field.................................2 21

14. Overlay of COMSOL Model and actual thruster in operation................................................2 22

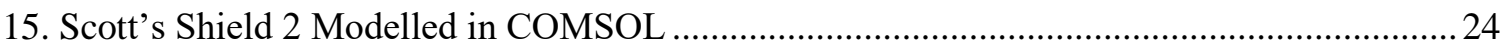

16. Radial Magnetic Field at the Ionization Chamber Wall and Halfway From Centerline to

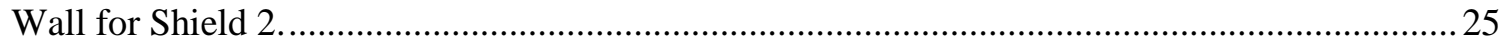

17. Magnetic Field Strength $(|\mathrm{B}|)$ at Ionization Chamber Wall and Halfway from Centerline

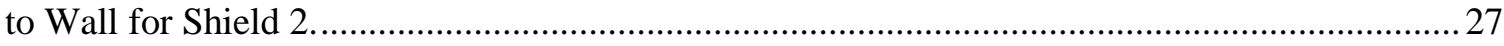

18. Radial Magnetic Field at Ionization Chamber Wall and Halfway from Centerline to Wall

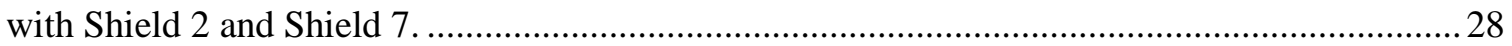

19. Magnetic Field Strength $(|\mathrm{B}|)$ at Ionization Chamber Wall and Halfway from Centerline to Wall for Shield 2 and Shield 7.

20. Magnetic Shields Designed in COMSOL for Possible Testing. Shield 10, 11, 12, 13, 14, and 15 from top left to bottom right.

21. Radial Magnetic Field and Magnetic Field Strength for Various New Shield Designs

Along the Thruster's Ionization Chamber Wall and Halfway from the Wall to the Centerline. ... 31

22. Exploded View of the RPA CREO Model and 2 Images of the Actual RPA Used for

Testing. 34

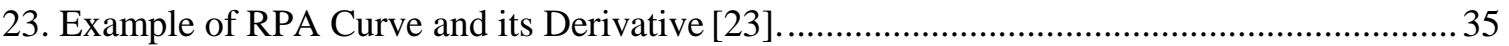

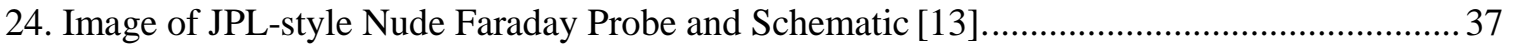

25. High Vacuum Equipment Corporation (HVEC) 350 Liter Bell Jar Chamber. .42

26. HVEC Vacuum System Schematic. Schematic Courtesy of Scott McGrail [15], 


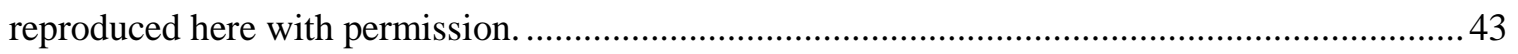

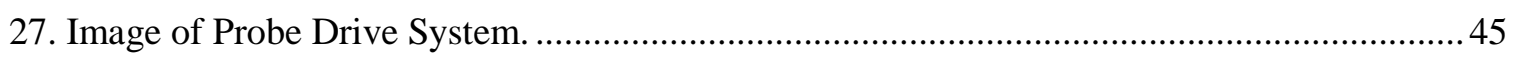

28. Electrical Diagram of RPA Collector and Faraday Probe. .................................................. 47

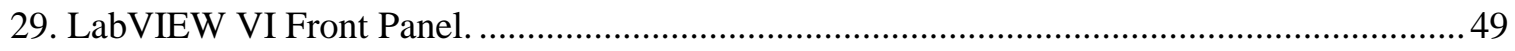

30. Electrical Schematic for HEMPT Testing. …............................................................... 51

31. Propellant Feed System Schematic for HEMPT and Hollow Cathode [15] ...........................53

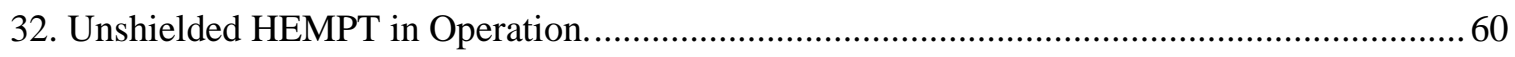

33. Unshielded HEMPT Ion Current Plume Profile with Faraday Probe....................................... 62

34. Unshielded HEMPT Ion Current Plume Profile with RPA. ..................................................63 63

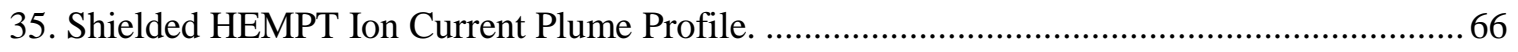

36. String Potentiometer Position Vs. Time for the Unshielded HEMPT. ....................................67

37. Hollow Cathode Electrical Connection Points....................................................................... 79

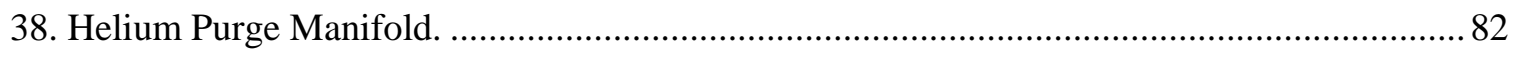

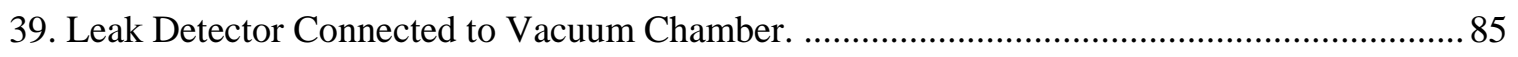

40. Components Needed for Connecting Leak Detector to Chamber........................................... 85

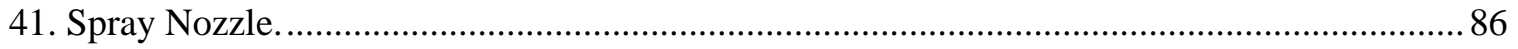

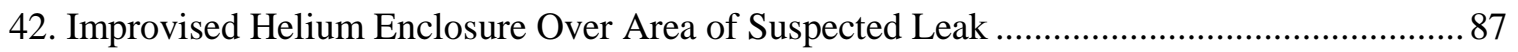

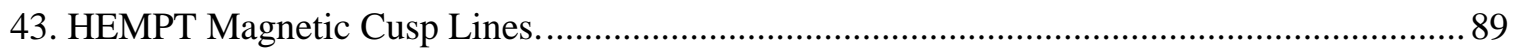

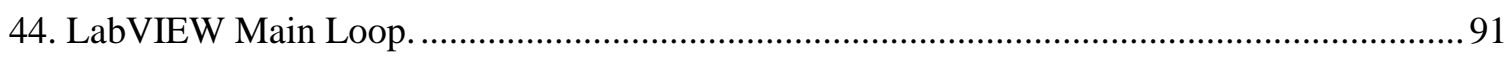

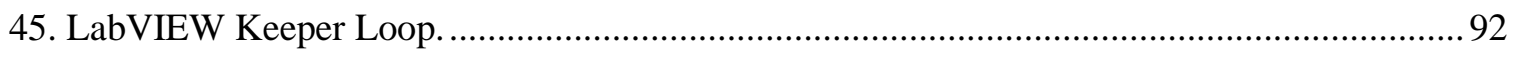




\section{LIST OF ACRONYMS}

$\begin{array}{ll}\text { CEX } & \text { Charge Exchange } \\ \text { CHT } & \text { Cylindrical Hall Thruster } \\ \text { DAQ } & \text { Data Acquisition System } \\ \text { EP } & \text { Electric Propulsion } \\ \text { HEMPT } & \text { High Efficiency Multistage Plasma Thruster } \\ \text { HVEC } & \text { High Vacuum Equipment Corporation } \\ \text { JPL } & \text { Jet Propulsion Laboratory } \\ \text { PPM } & \text { Permanent Periodic Magnet } \\ \text { PPPL } & \text { Princeton Plasma Physics Laboratory } \\ \text { RPA } & \text { Retarding Potential Analyzer } \\ \text { SCCM } & \text { Standard Cubic Centimeters per Minute } \\ \text { TWT } & \text { Travelling Wave Tube } \\ \text { UHP } & \text { Ultra High Purity }\end{array}$




\section{LIST OF SYMBOLS}

$A \quad$ Open area of the thruster [m²]

$A_{e} \quad$ Effective collection area of the RPA [m^2]

$A_{R P A} \quad$ Area of the RPA probe orifice [m²]

a Acceleration $\left[\mathrm{m} / \mathrm{s}^{\wedge} 2\right]$

c Effective exhaust velocity $[\mathrm{m} / \mathrm{s}]$

$e \quad$ Elementary charge $[1.6022 \times \llbracket 10 \rrbracket \wedge(-19)$ C]

F $\quad$ Force [N]

$f(V)$ Ion voltage distribution function

$g_{0} \quad$ Gravitational acceleration at sea level $\left[\mathrm{m} / \mathrm{s}^{\wedge} 2\right]$

I Impulse $[\mathrm{N} \cdot \mathrm{s}]$

$I_{b} \quad$ Beam current $[\mathrm{A}]$

$I_{c} \quad$ Average collected ion current $[\mathrm{A}]$

$I_{d} \quad$ Discharge current [A]

$I_{\text {probe }}$ Current collected by the probe [A]

$I_{s p} \quad$ Specific impulse [s]

$\frac{I^{++}}{I^{+}} \quad$ Fraction of doubly charged ions in the beam

$\left(\frac{j}{j_{0}}\right)_{A r^{+}}$CEX correction factor for singly charged ions

$M \quad$ Mass of an ion [kg]

$M_{f} \quad$ Final mass of spacecraft $[\mathrm{kg}]$

$M_{0} \quad$ Initial mass of spacecraft $[\mathrm{kg}]$

$M_{A r} \quad$ Mass of single Argon ion [6.6335× 『10』^(-26) kg]

$m \quad$ Mass $[\mathrm{kg}]$

$\dot{m} \quad$ Mass flow rate $[\mathrm{kg} / \mathrm{s}]$

$\dot{m}_{p} \quad$ Propellant mass flow rate $[\mathrm{kg} / \mathrm{s}]$

$n_{i} \quad$ Ion density [particles $/ \mathrm{m}^{\wedge} 2$ ]

$n_{0} \quad$ Neutral density [particles/m²]

$P \quad$ Chamber pressure [Torr]

$P_{k} \quad$ Keeper power [W]

$Q_{\text {ingested }} \quad$ Ingested volumetric flow rate [SCCM]

$Q_{\text {injected }} \quad$ Injected volumetric flow rate [SCCM] 
$Q \quad$ Total volumetric flow rate [SCCM]

$r \quad$ Radial distance from thruster centerline [m]

$T \quad$ Thrust $[\mathrm{N}]$, Temperature $[\mathrm{K}]$

$T_{g} \quad$ Grid transparency

$t \quad$ Time $[\mathrm{s}]$

$V_{b} \quad$ Beam voltage [V]

$V_{d} \quad$ Discharge voltage [V]

$V_{\text {probe }}$ Discriminator grid potential [V]

$V_{R P A} \quad$ Most probably ion voltage [V]

$Z_{i} \quad$ Ion charge state

$z \quad$ Axial distance from thruster to probe [m]

$\alpha \quad$ Thrust correction factor

$\alpha_{m} \quad$ Double ion correction factor for mass utilization efficiency

$\gamma \quad$ Total thrust correction factor

$\Delta V \quad$ Change in spacecraft velocity $[\mathrm{m} / \mathrm{s}]$

$\eta_{b} \quad$ Beam current fraction of discharge

$\eta_{c} \quad$ Conductance correction factor

$\eta_{m} \quad$ Mass utilization efficiency

$\eta_{0} \quad$ Electrical efficiency

$\eta_{T} \quad$ Total thruster efficiency

$\eta_{v} \quad$ Beam voltage fraction of discharge

$\theta \quad$ Beam divergence half-angle

$\sigma_{1} \quad$ Effective cross sectional area of $\mathrm{Ar}+\mathrm{Ar} \mathrm{CEX}$ interaction [ $\left[\AA^{\wedge} 2\right]$

$\phi_{p} \quad$ Plasma potential [V] 


\section{INTRODUCTION}

Electric propulsion systems, in contrast to their chemical counterparts, provide a massefficient means of generating small levels of thrust for long durations. The total energy produced by a traditional chemical rocket engine is limited to the energy contained within the propellants. Electric propulsion systems, however, have no such theoretical energy limit. It is possible to achieve very high exhaust velocities in exchange for high power consumption. With such highefficiency propulsion systems, spacecraft designers are able to incorporate larger, more capable payloads onto smaller busses. However, EP still has plenty of room for improvement. This thesis seeks to advance a relatively new class of EP thrusters with promising performance characteristics.

The underlying goal of this thesis is to reduce the hollow exit plume of a High-Efficiency Multistage Plasma Thruster (HEMPT) through the design, manufacture, and test of a magnetic shield. The HEMPT currently exhibits low ion current along the centerline of its plume. This is due to its magnetic field, which diverges at the exit. This causes ions to accelerate from the thruster exit at significant, non-zero angles. A Faraday probe will be used to measure and characterize the ion current of the thruster plume at various points both with and without a shield. Simultaneously, a Retarding Potential Analyzer (RPA) will be used to measure the ion energy distribution of the plume. 


\section{ELECTRIC PROPULSION BACKGROUND}

\subsection{THRUSTER BASICS}

The basic operation of an electric thruster is very similar to that of a traditional chemical rocket thruster: In both types of thrusters, mass is accelerated and ejected from the thruster's exit. This ejected mass imparts a net force on the spacecraft known as thrust. In both cases, propellant must be carried on-board the spacecraft until it is expended to produce thrust. The rocket equation describes the relationship between the spacecraft change in velocity $(\Delta V)$ and expended propellant mass and is derived starting with Newton's second law:

$$
F=m a
$$

Plugging in for the spacecraft parameters, Eqn. 1 becomes

$$
T=M \frac{d v}{d t}
$$

where $T$ is the force on the spacecraft (thrust), $M$ is the vehicle mass, and $v$ is the spacecraft velocity. Also, the force on the spacecraft is equal and opposite to the time rate of change of the propellant's momentum. This is shown in Eqn. 3,

$$
T=-\frac{d}{d t}\left(m_{p} v_{e x}\right)
$$

where $m_{p}$ is the mass of the ejected propellant and $v_{e x}$ is the propellant exhaust velocity.

Assuming $v_{e x}$ is constant with respect to time, Eqn. 3 can be rewritten as

$$
T=-v_{e x} \frac{d m_{p}}{d t}
$$

Because the only change in mass of the spacecraft is the propellant being ejected, $\frac{d m_{p}}{d t}$

can be rewritten as $\frac{d M}{d t}$, the time rate of change of the total spacecraft mass. Equating Eqn. 2 and Eqn. 4 results in

$$
M \frac{d v}{d t}=-v_{e x} \frac{d M}{d t}
$$

which can be rearranged to yield

$$
d v=-v_{e x} \frac{d M}{M}
$$


Both sides of Eqn. 6 are then integrated to net

$$
\Delta V=v_{e x} \ln \left(\frac{m_{d}+m_{p}}{m_{d}}\right)
$$

where $m_{d}$ is the dry mass of the vehicle, which is defined as the total vehicle mass minus the propellant mass [1].

Specific impulse is defined as the total impulse divided by the unit weight of propellant.

It is a measure of rocket mass efficiency, expressed in units of seconds and given by

$$
I_{s p}=\frac{T}{\dot{m} g_{0}}=\frac{v_{e x}}{g_{0}},
$$

where $I_{s p}$ is specific impulse and $g_{0}$ is sea-level acceleration due to gravity. Therefore, Eqn. 7 can be expressed in terms of $I_{s p}$ as

$$
\Delta V=I_{s p} g_{0} \ln \left(\frac{m_{d}+m_{p}}{m_{d}}\right)=I_{s p} g_{0} \ln \left(\frac{M_{0}}{M_{f}}\right),
$$

where $M_{0}$ is the initial mass and $M_{f}$ is the final mass [1].

Traditional rocket engines harness chemical energy to create rapidly expanding hot gasses. These gasses are funneled through a nozzle to accelerate them, producing thrust. This results in high thrust levels with relatively low specific impulses. In contrast, EP utilizes neutral, inert, gaseous propellants which are ionized and electrostatically accelerated from a thruster. A more in-depth look at the principles of EP thrusters is provided in the following section.

\subsection{ELECTRIC PROPULSION BASICS}

The main benefit of electric propulsion compared to its chemical counterpart is its mass efficiency. Chemical rocket engines can achieve an $I_{s p}$ of 450s [2]. Electric propulsion technologies have much higher limits, with ion thrusters and hall thrusters generally operating in $I_{s p}$ ranges of 2000-4000s and 1000-2000s, respectively [3].

There are many types of electric spacecraft propulsion technologies, including electrothermal, electrostatic, and electromagnetic. Two of the most common in use today are Hall 
Effect Thrusters (HETs) and gridded ion thrusters (GITs), which are both electrostatic thrusters. In a sense, HEMPTs can be thought of as a hybrid between these two thruster technologies, so understanding of HETs and GITs can be applied directly to HEMPTs.

The body of a gridded ion thruster is generally a hollow tapered cylinder which forms the ionization chamber (see Figure 2).

Neutral, gaseous propellant (Xenon, in most cases) is injected into the chamber at the narrow end. A cathode is also placed at the narrow end. The cathode emits electrons which collide with the neutral gas atoms and liberate electrons,

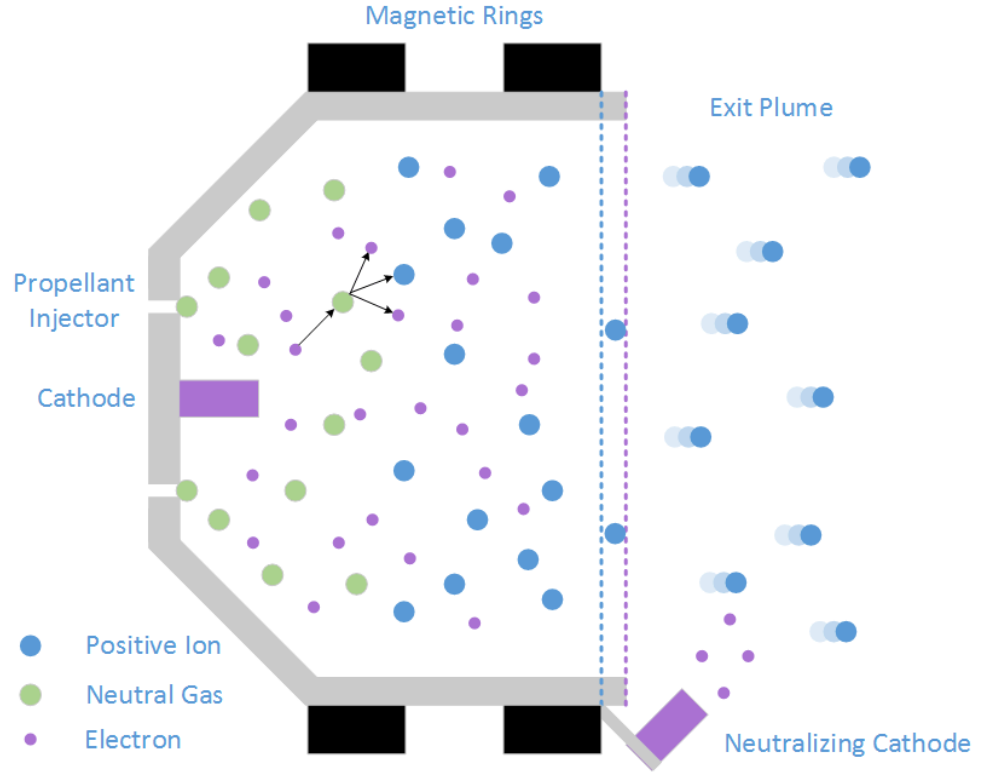

Figure 1. Gridded Ion Thruster Operation. creating positively charged ions. A series of magnetic rings around the circumference of the thruster contain the plasma and minimize ion contact with the chamber walls. The ions eventually drift towards the exit of the thruster which is blocked by a series of grids. The grids are biased such that ions which pass through the grids are electrostatically

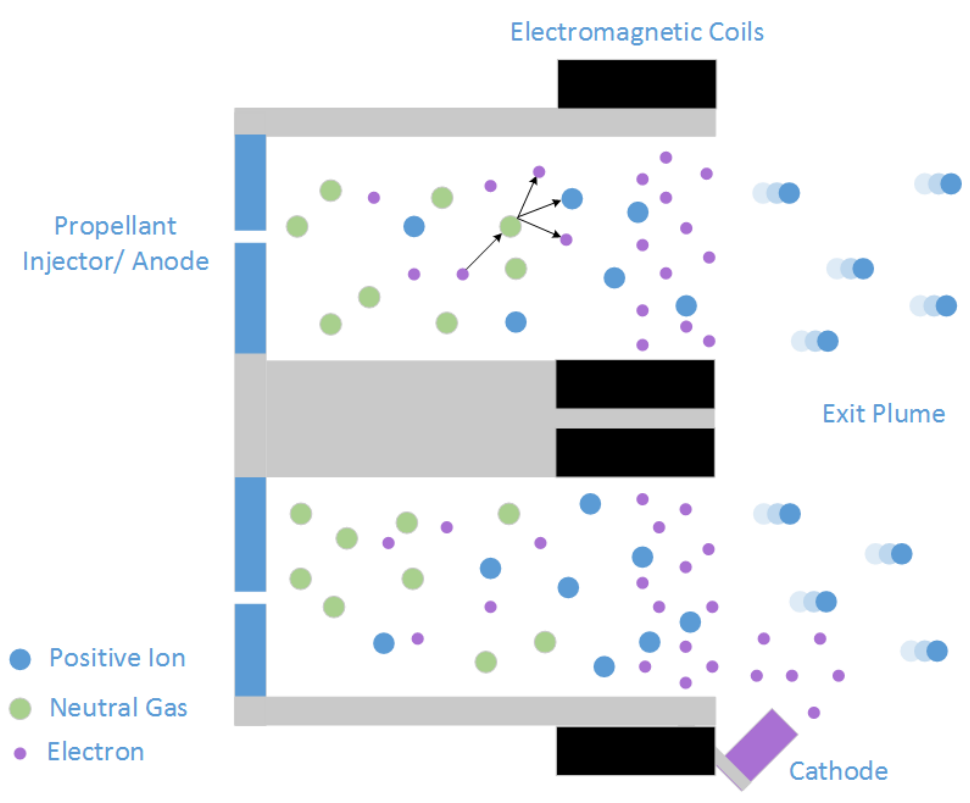

Figure 2. ALT Hall Thruster Operation. 
accelerated to very high velocities before being ejected. The ejected ion beam is then neutralized via a second cathode which injects electrons into the charged plume [3].

Hall thrusters, in many ways, are quite similar to ion thrusters. However, there are some defining differences. Firstly, their structure is necessarily distinct. Hall thrusters are generally cylindrical in shape. However, instead of being completely hollow, there is a center cylinder which serves as one pole of an electromagnet. The other pole is wrapped around the outer wall of the chamber. Between the two poles the magnetic field is mostly radial. Another important difference is the complete absence of grids in a Hall thruster and the need for only a single cathode. Without grids, ion acceleration is achieved via an electric field generated by an anode at the upstream wall of the thruster. Electrons from the cathode flow into the thruster's ionization chamber where they are trapped in the thruster's magnetic field. They spiral around the central cylinder, creating regions of high negative charge. When a neutral gas atom passes through these regions, there is a high chance of electron impact which results in ionization. These newly created ions are then accelerated by the electric field generated by the anode, creating thrust. Just like in ion thrusters, the exit plume is neutralized by pumping electrons into it. Figure 1 shows a diagram of the operation of an anode layer Hall thruster [3]. 


\section{HEMPT BACKGROUND}

\subsection{HEMPT BASICS}

Hall effect thrusters and gridded ion thrusters have been around since the late 1950s. Here, a relatively new electric thruster is discussed. It was conceptualized in 1998. Engineers at Thales Electron Devices developed the concept over the next several years into the high efficiency multistage plasma thruster, or HEMPT. It can operate over a wide range of thrust values, from a few hundred $\mu \mathrm{N}$ to over $150 \mathrm{mN}$. It is considered "high efficiency" because of its ability to convert more than $80 \%$ of electric power into kinetic energy of the ion beam it produces. The "multistage" portion of its name is attributed to its multicusp magnetic design. The HEMPT's multiple magnetic cusps are a design borrowed from travelling wave tubes (TWT). TWTs use permanent periodic magnet systems to focus electron beams and are commonly used on spacecraft to amplify RF signals [4].

HEMP thrusters offer promising solutions to many unsolved problems of HETs and GITs. Specifically, Hall effect thrusters are life-limited by channel wall erosion, while gridded ion thrusters typically fail when the high-energy plasma erodes their acceleration grids [1]. The HEMPT is not prone to either of these problems because its cusped magnetic field design forces the plasma off of the walls substantially reducing wall erosion (see Appendix C), and it operates without grids. Reducing plasma contact with the walls is important to maintain thermal efficiency. HEMPTs can exhibit thermal losses of 10-20\% while thermal losses in a Hall effect thruster are often $30-45 \%$ [5]. 


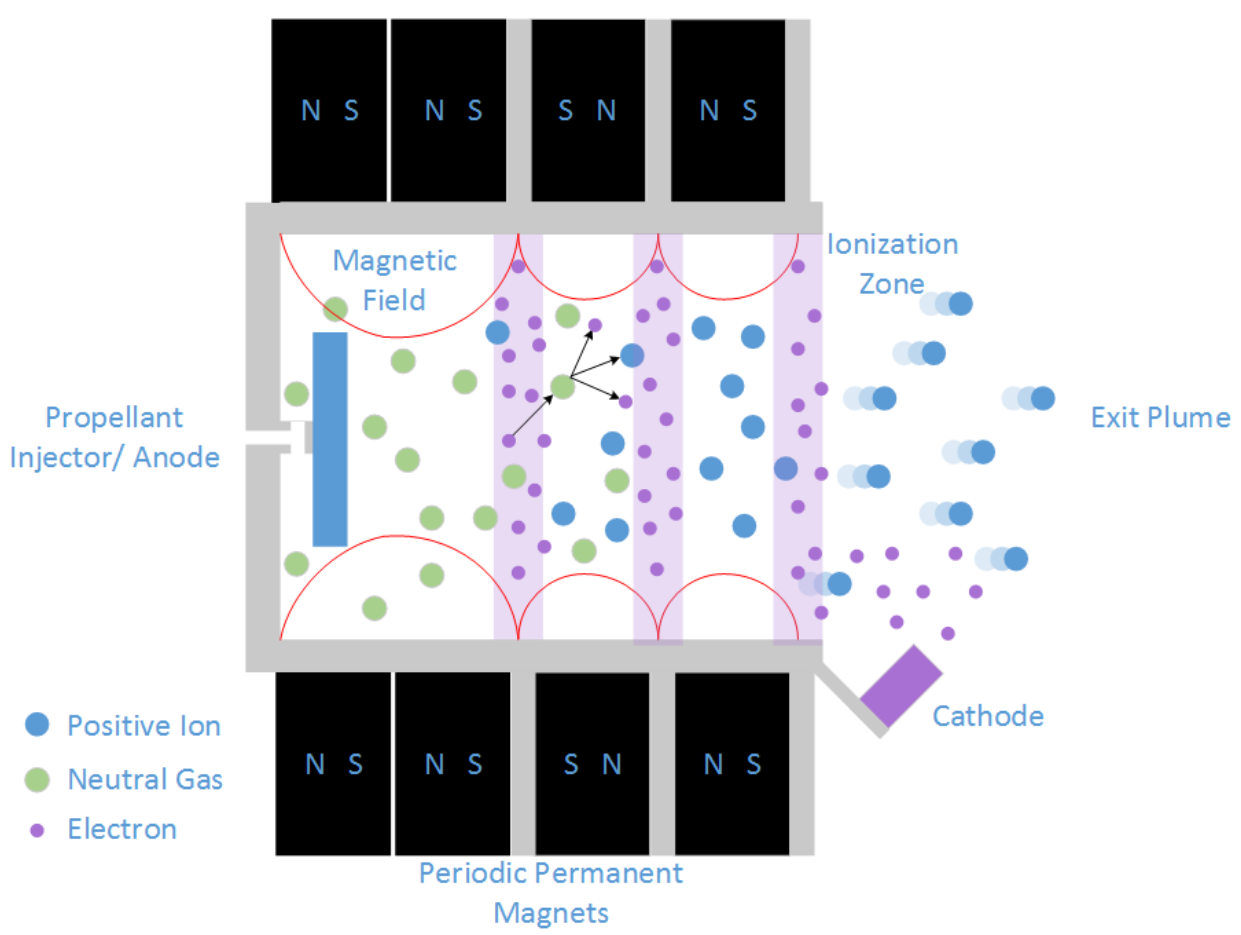

Figure 3. High Efficiency Multistage Plasma Thruster Operation.

The physical construction of a HEMPT is relatively simple. The thruster is built around a ceramic tube which is surrounded by rings of permanent magnets. The ceramic tube serves as the walls of the ionization chamber, and the magnets create the cusped field regions which trap the electrons on closed drift orbits [5]. The HEMPT also has a high voltage feedthrough to an anode, which provides the electric field to pull electrons into the discharge chamber and push ions out of the exit. A propellant feedthrough line is used to both support the anode and flow gas (generally Xenon) into the chamber. A hollow cathode sits outside of the thruster. It provides electrons to both ionize propellant and neutralize the exhaust ion beam. Figure 3 shows a schematic of the HEMPT.

Operation of a HEMPT is very similar to that of a hall or ion thruster. First, propellant enters the ionization chamber via the propellant injector. Simultaneously, the cathode downstream of the thruster produces electrons which are attracted to the anode inside of the thruster. Electrons, aided by collisions with propellant, ions, and other electrons, are able to migrate up the thruster towards the positively charged anode. The closer the magnetic "cell" is to 
the anode, the higher the electron and plasma densities. Most of these electrons become trapped in $\mathrm{ExB}$ drifts at the cusped regions of the magnetic field generated by the periodic permanent magnets. This is because the magnetic field is almost entirely radial at these locations. As neutral propellant gas passes through these regions, electrons impact the gas atoms, resulting in positively charged ions and additional electrons. These cusped field regions become negatively charged and accelerate positive ions that pass through them. The closer the magnetic "cell" is to the anode, the higher the electron and plasma density [5].

In terms of performance, early experiments by Thales Electron Devices show that the HEMPT is competitive with traditional electric propulsion technologies [6]. This is true even though the HEMPT has not been fully developed to its flight-ready state. For example, the BB HEMP 3050 DM7 thruster (tested at the University of Giessen) performs in an $\mathrm{I}_{\mathrm{sp}}$ range of 5003500s and a thrust range of $200 \mu \mathrm{N}-150 \mathrm{mN}$, depending heavily on anode voltage and propellant flow rate. Generally, increasing anode voltage and propellant flow rate improves performance, and the HEMPT is very throttleable and capable of operation at a wide range of set points [6]. For comparison, ion thrusters perform in the $\mathrm{I}_{\mathrm{sp}}$ range of 2000-4000s and Hall thrusters operate with an $\mathrm{I}_{\mathrm{sp}}$ range of 1000-2000s [3].

\subsection{HEMPT DIMENSIONS AND HOLLOW CATHODE}

The HEMPT under test for this thesis is a research model built and donated by JPL. It is a stainless steel thruster body with a stainless steel anode. The ionization channel is a 1" inner diameter alumina tube ( $99.6 \%$ pure) which is 2.5 " in length. It uses a total of 40 Samarium Cobalt magnets arranged in 4 rings around the alumina tube (10 magnets per ring). The magnets measure $0.5 \times 0.4 \times 0.2$ " and have a nominal surface magnetic flux density of $300 \mathrm{mT}$. Because the magnets are so strong, they tend to push against each other and jump out of their mountings, so high temperature wire was wrapped around the magnets to contain them. A propellant feed line is 
connected to the back of the thruster and feeds through to the anode. The thruster body is electrically isolated from the anode via a ceramic connector.

The hollow cathode used in this experiment has a 0.25 " diameter insert and was also built at JPL and donated for research at Cal Poly. A hollow cathode's main structure is a hollow tube with a small orifice plate at the end. The tube has a cylindrical insert, which serves as the electron emitter, that is placed against the orifice plate. The outside of the cathode tube is wrapped with a heater which is capable of heating the insert to a temperature sufficient for electron emission.

Once heated, gas is passed over the insert and becomes ionized by the emitted electrons, forming a plasma in the insert region of the hollow cathode. Electrons are then extracted from this plasma via the orifice. A keeper electrode is placed around the entire assembly. The keeper is responsible for turning on the cathode discharge, maintaining cathode temperature and operation, and protecting the orifice and heater from ion bombardment [1]. A schematic of this assembly is shown in Figure 4. Both the HEMPT and hollow cathode are shown in Figure 5 in their test fixture. Copper sheets were mounted to the body of the HEMPT to conduct heat away from thruster and radiate it to the chamber. The HEMPT body was electrically isolated from the chamber, so thermal conduction was not a viable option for heat rejection.

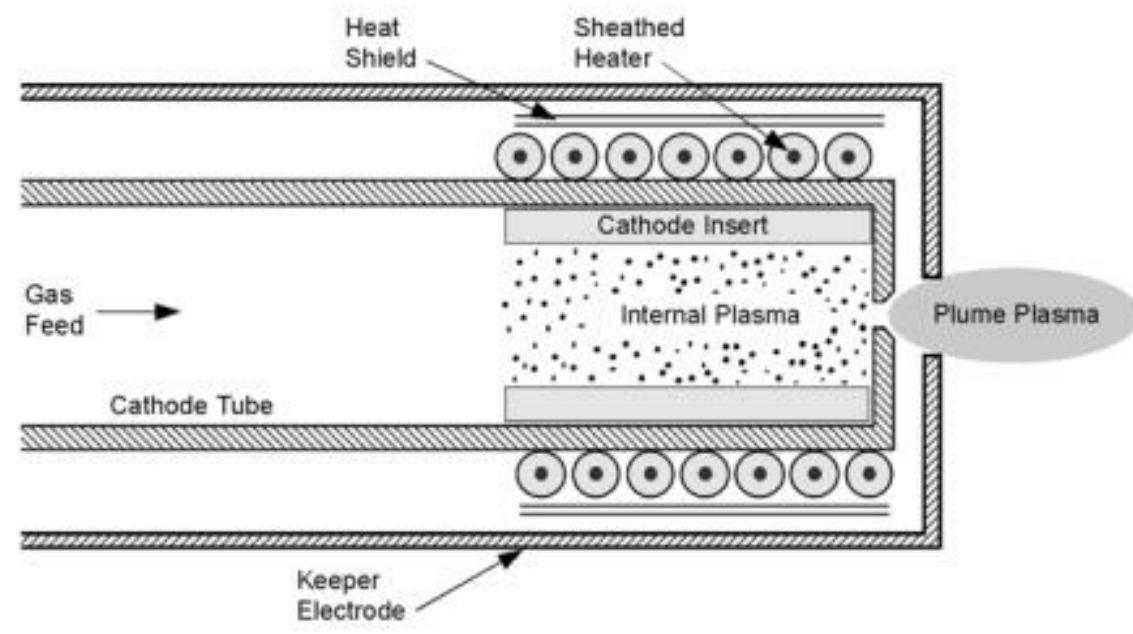

Figure 4. Hollow Cathode Assembly Schematic [1]. 


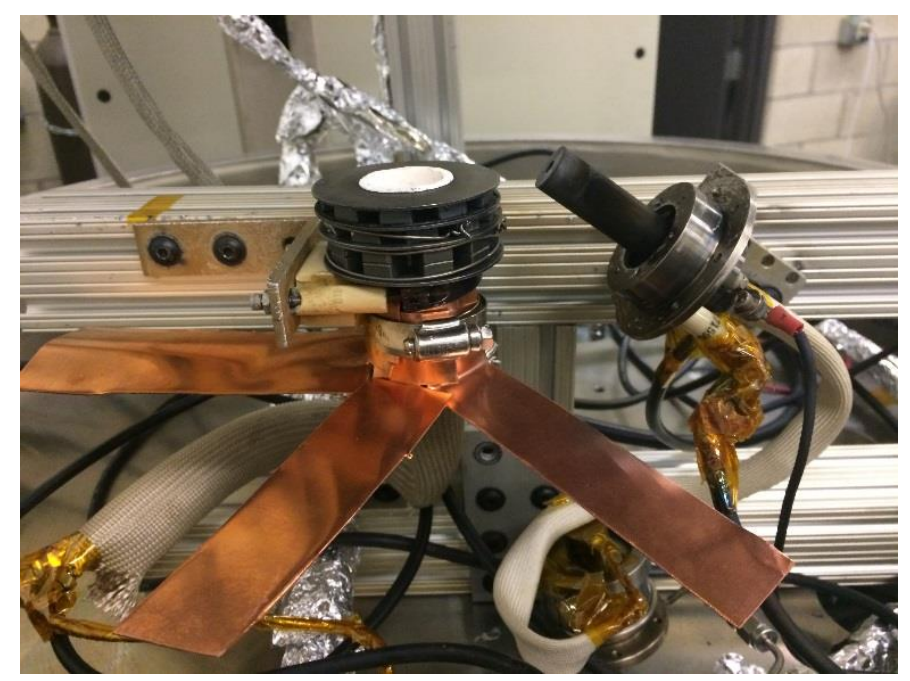

Figure 5. Image of HEMPT and Hollow Cathode. The HEMPT is shown on the left and the hollow cathode is on the right.

\subsection{HOLLOW PLUME}

HEMP thrusters have many benefits compared to other EP thrusters. However, the same magnetic rings which provide many of these benefits also create a major problem. The ring of magnets located at the thruster's exit plane creates a magnetic field which wraps around the outside of the thruster. This tends to pull the exit plume away from the center axis of the thruster, creating a diverging, hollow plume. This means that the peak ion current occurs at some angle off of the centerline. Essentially, a significant fraction of the exiting ions are being ejected from the thruster at angles that aren't parallel with the thruster centerline, which negatively impacts thrust and efficiency. This divergent nature of the plume is clearly visible in Figure 6. 


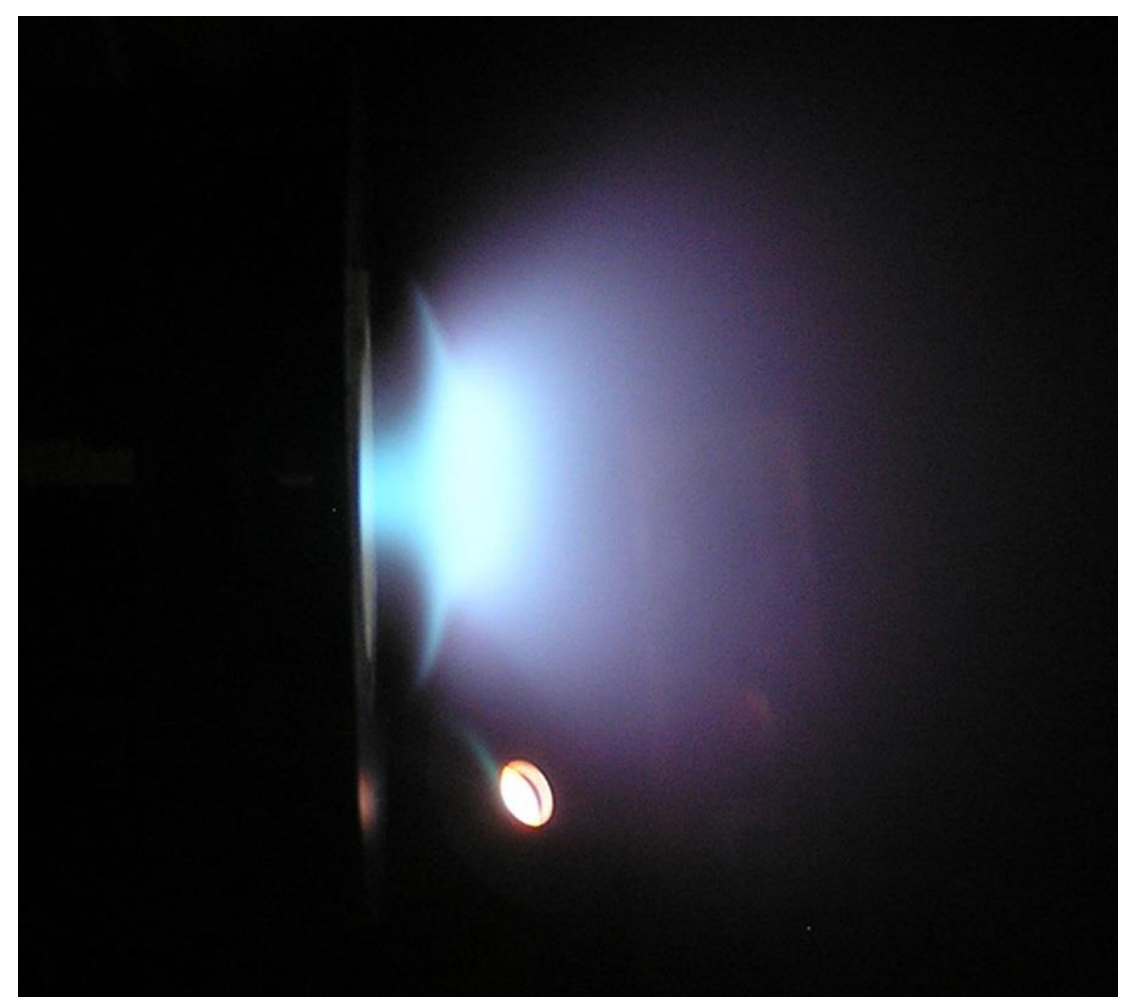

Figure 6. Side view of HEMPT during operation at JPL [7].

HEMPTs are not the only thrusters which experience this divergent plume phenomenon. Both Hall thrusters and diverging cusped field thrusters (DCFTs) have similar issues. For example, Raitses et al. at the Princeton Plasma Physics Laboratory observed significant plume divergence on a permanent magnet cylindrical hall thruster. More notably, they were able to successfully and substantially reduce the divergent nature of the plume. This resulted in much greater ion current at or near the thruster's centerline which resulted in an increase in efficiency of about $28 \%$.

\subsection{PROPOSED SOLUTION AND PREVIOUS WORK}

The research done by Raitses et al. at the PPPL on divergent CHT plumes is what motivates the proposed solution to the similar problem seen with the HEMPT. The researchers at the PPPL used magnetic shields manufactured from low-carbon steel to modify the exit plume of 
their thruster. The same procedure will be applied to the Cal Poly HEMPT with the ultimate goal of reducing the divergence angle of the exit plume and positively affecting thruster performance.

Scott McGrail, who graduated Cal Poly with his MS in Aerospace Engineering in 2013, performed significant work towards this goal for his thesis. First, he built a retarding potential analyzer (RPA) to measure the ion energy distribution of the thruster plume. He also assembled a basic test stand and linear drive system to sweep the RPA across the plume. Through magnetic simulation, he designed a shield in an attempt to remedy the hollow plume. Testing the shielded HEMPT showed that the plume divergence was decreased by the added shield. Unfortunately, losses were taken to predicted thrust, $\mathrm{I}_{\mathrm{sp}}$, and total efficiency (these quantities were not directly measured, but calculated based off of other measurements, explained later). Using further magnetic simulation he predicted that the internal magnetic field of the thruster was adversely affected by the addition of the magnetic shield, resulting in a decrease in performance. He also suggested a shield design which may offer a reasonable compromise between altering the internal magnetic field and reducing the hollow characteristic of the plume.

Here, the work Scott started will be verified and furthered. The HEMPT will be characterized again and the shield he suggested will be manufactured and tested. Results from these tests will be compared to the results from the unshielded HEMPT, as well as the results from the shield he tested. 


\section{2D MAGNETIC FIELD MODELING}

\subsection{MODELING CONSTRAINTS}

The first step to solving almost any engineering problem is to create a model to provide a basis for understanding the issues. In this case, a 2D model of the HEMPT was created in COMSOL, a multiphysics simulation program. The thruster's physical dimensions were measured and a cross-section view was created in the program. This cross-section is the result of a plane slicing the thruster such that the thruster's centerline is contained by the plane. The thruster is quite nearly axisymmetric, so using a 2D slice through the centerline should theoretically provide a reasonably accurate model. Next, material properties were added to each part of the thruster. Specifically, the relative permeability of each part of the HEMPT was assigned in the model. The specific values and material types that were used in this model are summarized in Table 1.

Table 1. Material Properties Used in HEMPT Physics Model

\begin{tabular}{lll}
\hline Material Name & Part Name & Relative Permeability $\left(\mu_{r}\right)$ \\
\hline Vacuum & Background & 1 \\
Aluminum Oxide & Ionization Chamber Wall & $1[8]$ \\
Iron & Magnet Separators & $5000[9]$ \\
Stainless Steel & Thruster Body & $100[9]$ \\
Stainless Steel & Anode & $100[9]$ \\
Samarium Cobalt & Magnets & $1.05[10]$ \\
\hline
\end{tabular}

Values in this table represent generally accepted values for these materials, or values for materials similar to these. The actual relative permeabilities of the various materials used in the HEMPT were not measured or verified.

\subsection{MODELING PROCESS}

The COMSOL AC/DC module with the Magnetic Fields (No Currents) package was used to simulate the magnetic field of the thruster. This was done by assigning remnant flux density to the model's permanent magnets. For this, the magnetic field strength of the magnets was 
measured using a Magnetic Instruments Model 2010 Serial Port Guassmeter. The samarium cobalt magnets had an average surface magnetization of $300 \mathrm{mT}$. This value was assigned to the permanent magnets in the $\mathrm{x}$-direction (parallel to the thruster centerline). The sign of the remnant flux density determined whether the north or south side of the permanent magnet was facing in the direction of the thruster exit. Just like in the actual HEMPT, the magnets closest to the exit had their south poles facing outward. The next ring of magnets had opposite polarity: north was facing towards the thruster exit. The final two rings of magnets were arranged in the same way as the $1^{\text {st }}$ ring.

Once the model's geometry and material properties were fully defined, a mesh was generated to facilitate solving the model.

COMSOL's built in automatic mesh generation

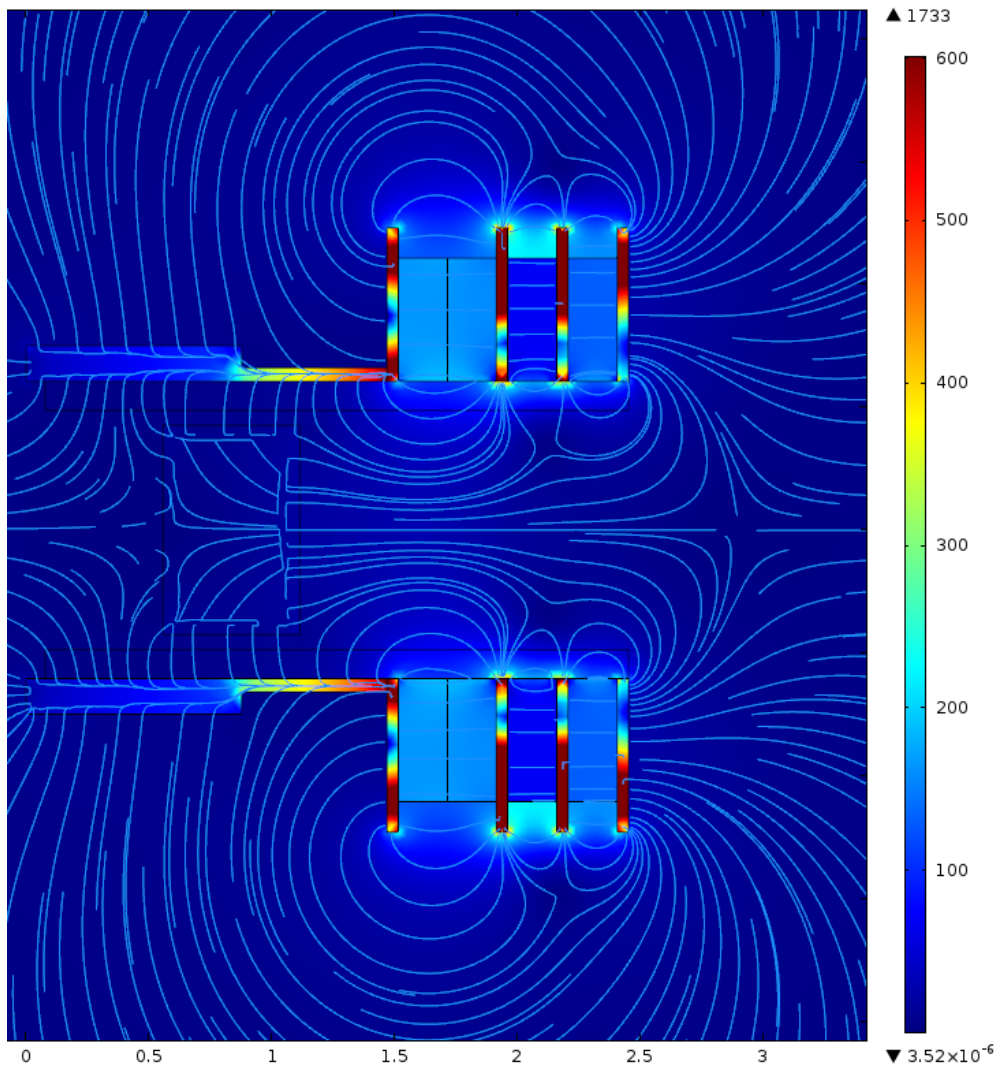
Figure 7. Surface Plot of the Norm of Magnetic Flux Density, $|\vec{B}|$, with Overlaid Streamlines of Magnetic Flux Density, $\overrightarrow{\boldsymbol{B}}$. The values on the color scale to the right of the image are the magnetic flux density norm expressed in $m T$.

was used here with "Extra Fine" element size. The study was then solved using the stationary (not time-varying) solver. A 2D surface plot of the norm of the magnetic flux density, $|\vec{B}|$, as well as a streamline plot of the magnetic flux density were created from the solution data. These are shown overlaid in Figure 7. 
This model was compared against the model produced by Scott McGrail for his thesis.

Scott's model, created using MagNet, is shown in Figure 8.

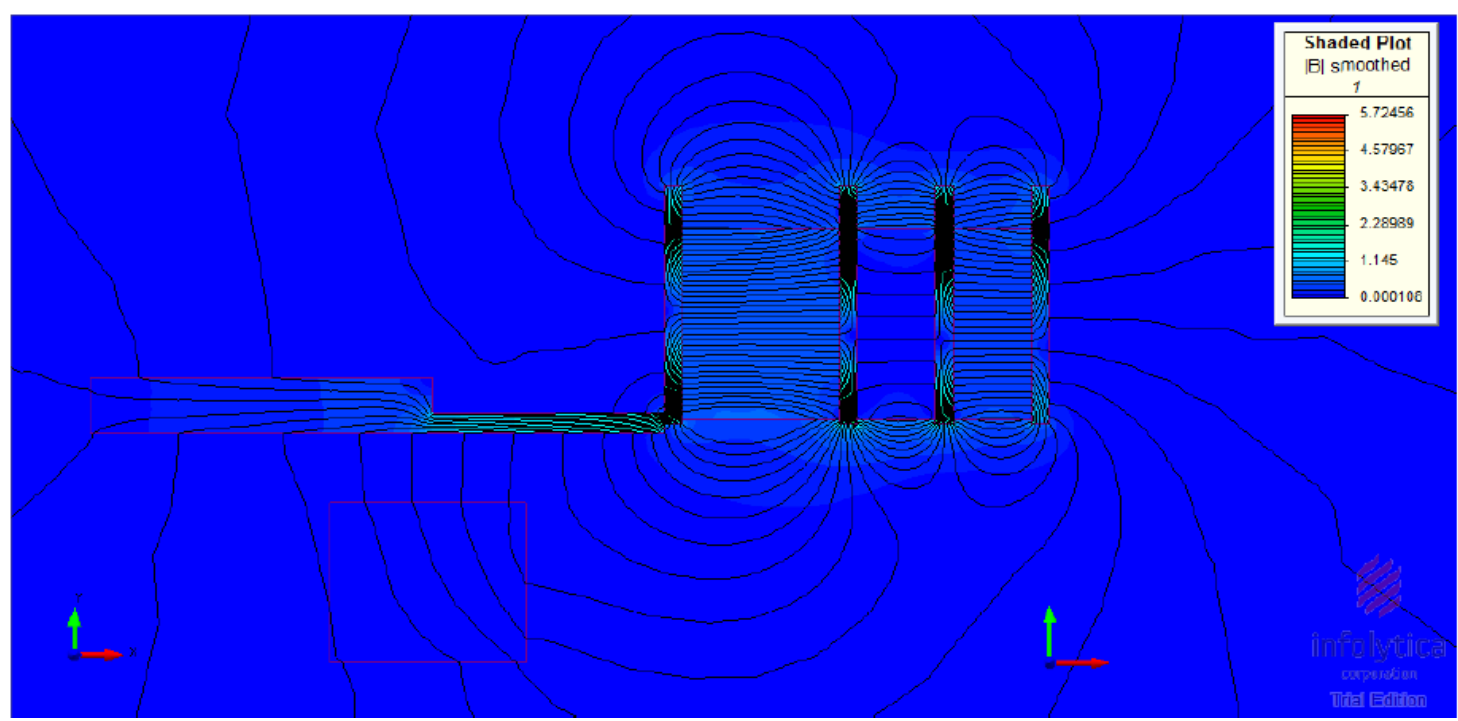

Figure 8. HEMPT Magnetic Model Produced in MagNet by Scott McGrail [15]. Image reproduced here with permission. The values on the color scale to the right of the image are the magnetic flux densitv norm expressed in Guass.

For comparison, both models are overlaid with transparency in Figure 9. This is useful for comparing the structure of the field lines. The white lines are from the COMSOL model and the black streamlines are from MagNet.

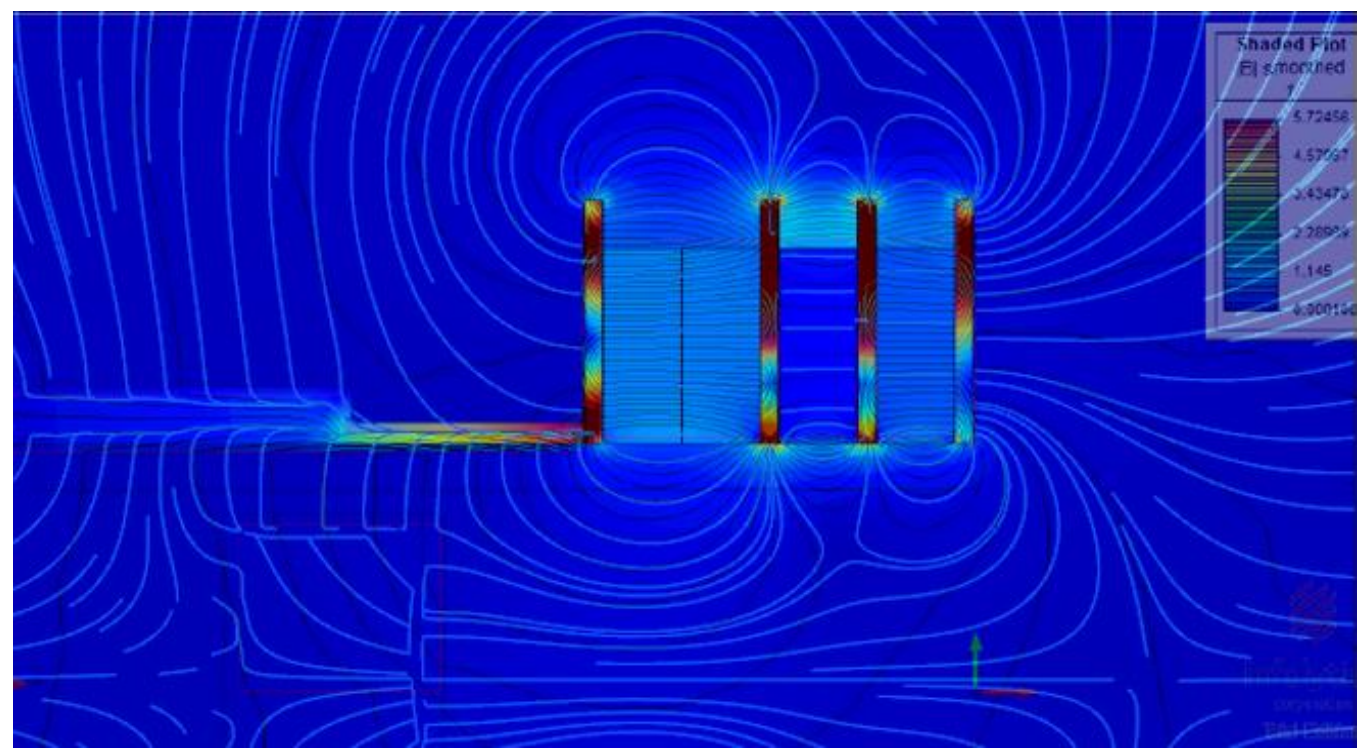

Figure 9. COMSOL HEMPT Magnetic Model Overlaid on MagNet Model. The color scale on the right applies to the MagNet model and is given in Guass. 
As expected, the models have a lot of similarities. The streamlines for each model are almost perfectly aligned in the majority of cases. Significant dissimilarities only occur near the centerline of the thruster. It's likely that the MagNet model was formed as a 2D model, without axial symmetry. This would allow the magnetic field streamlines to pass through the centerline unimpeded. Indeed, this is what the MagNet streamlines seem to do, whereas the COMSOL lines begin to bend and become mostly axial at the centerline. This behavior is expected from this thruster configuration: the magnetic field should be mostly axial at the centerline [4].

One other significant discrepancy is visible throughout the anode. The MagNet model shows little effect on the field streamlines due to the stainless steel anode. The COMSOL model of the anode seems to affect the lines in a much more significant way. This is most likely also attributable to the same cause as the previously discussed centerline issue. The stainless steel anode is located on the thruster's centerline, so extra compression of the field lines is expected within the anode due to the opposing magnets on either side of the centerline.

Another point to note about both models is the cusped zones of the magnetic field. The cusps are clearly visible at the lower edges of the iron magnet separators. As previously discussed, these cusped regions are critical to the operation of the HEMPT. Both models are in relative agreement on the geometry of the field at the cusps. Notably, both models show similar behavior of the exit cusp. This behavior is the main culprit of the thruster's divergent plume.

\subsection{MAGNETIC MODEL VALIDATION}

The COMSOL model created for this thesis was originally compared to the MagNet Model that Scott created for his thesis in order to validate it. However, agreeing with a previous model can only be so beneficial. It does not prove that either model is correct, only that they agree. The models could be equally far from the true behavior of the magnetic field within the physical thruster. For this reason, the magnetic field was measured at discrete points. Once again, the Magnetic Instrumentation Model 2010 Serial Port Guassmeters were used for this task. A 
transverse probe was used to measure the radial component of the field at each point while an axial version measured the axial component of the field.

In order to accurately control the location of the probes at the time of data collection, a simple 2-axis measurement apparatus was designed and manufactured, and is shown in Figure 10. Most of the apparatus was constructed with wood to minimize alterations to the measured field. Two platforms, one for the $y$-axis and one for the $x$-axis, were mounted to two $1 / 4$ inch diameter rails each. One rail allowed for free motion while the second was threaded. The platforms had two through holes drilled to allow the rails to pass through. Threaded T-nuts were press-fit into one of the through holes on each platform to allow the threaded rod to convey motion to the platform. By turning the cranks on the threaded rails the location of the probes relative to the thruster could be accurately controlled. The thruster's base is not flat, but rather has the propellant gas tube protruding from it. Therefore, a plastic cup with its base removed was fastened to the $\mathrm{x}$ axis platform with double-sided tape to act as an impromptu stand for the thruster.
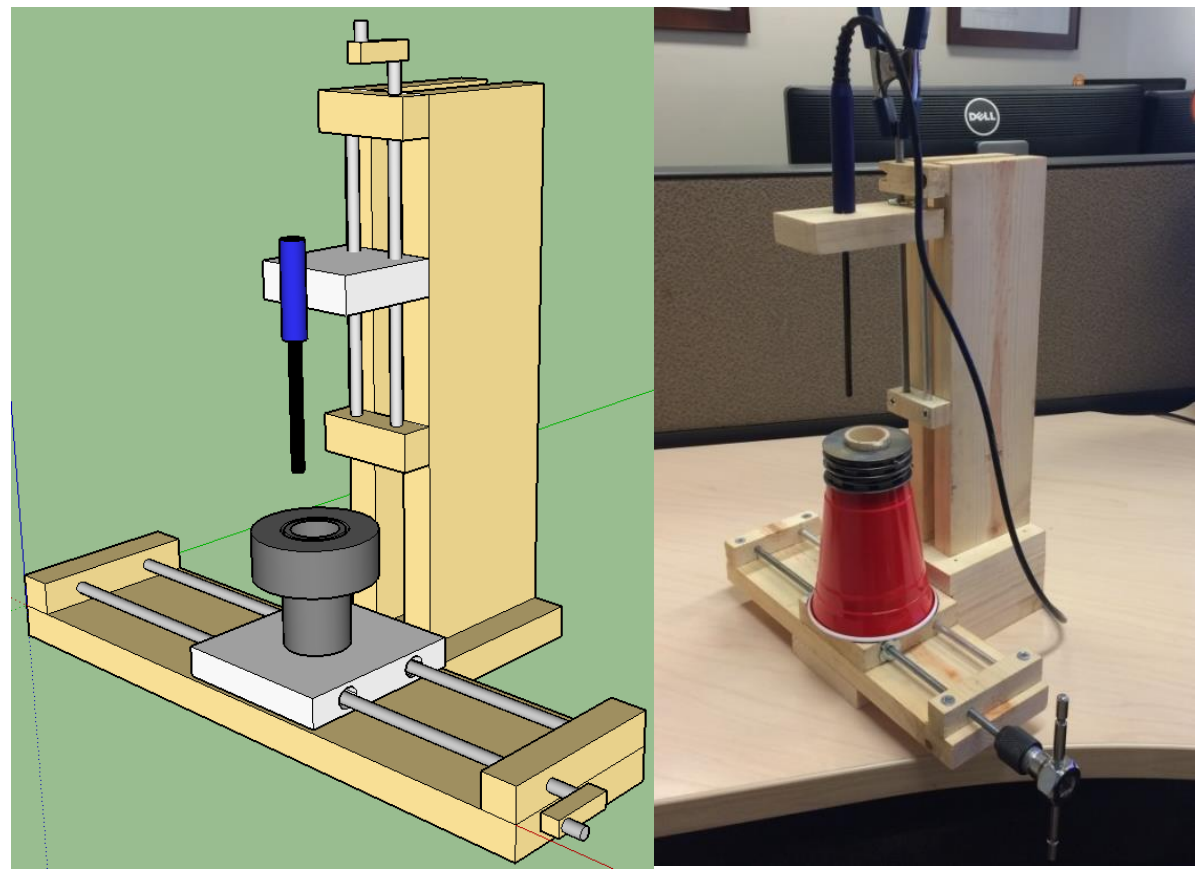

Figure 10. Magnetic Probe Stand Model and Implementation. 
The two components of the magnetic field were assembled, along with the locations at which the measurements were taken. This enabled the creation of an arrow surface plot, shown in Figure 11. This is analogous to the streamline plots presented previously but with discrete values rather than continuous streams. The directions of the arrows indicate the direction of the magnetic field (arrows point from north to south) at discrete points, and the size of the arrows indicates the relative magnitude of the magnetic field at that point.

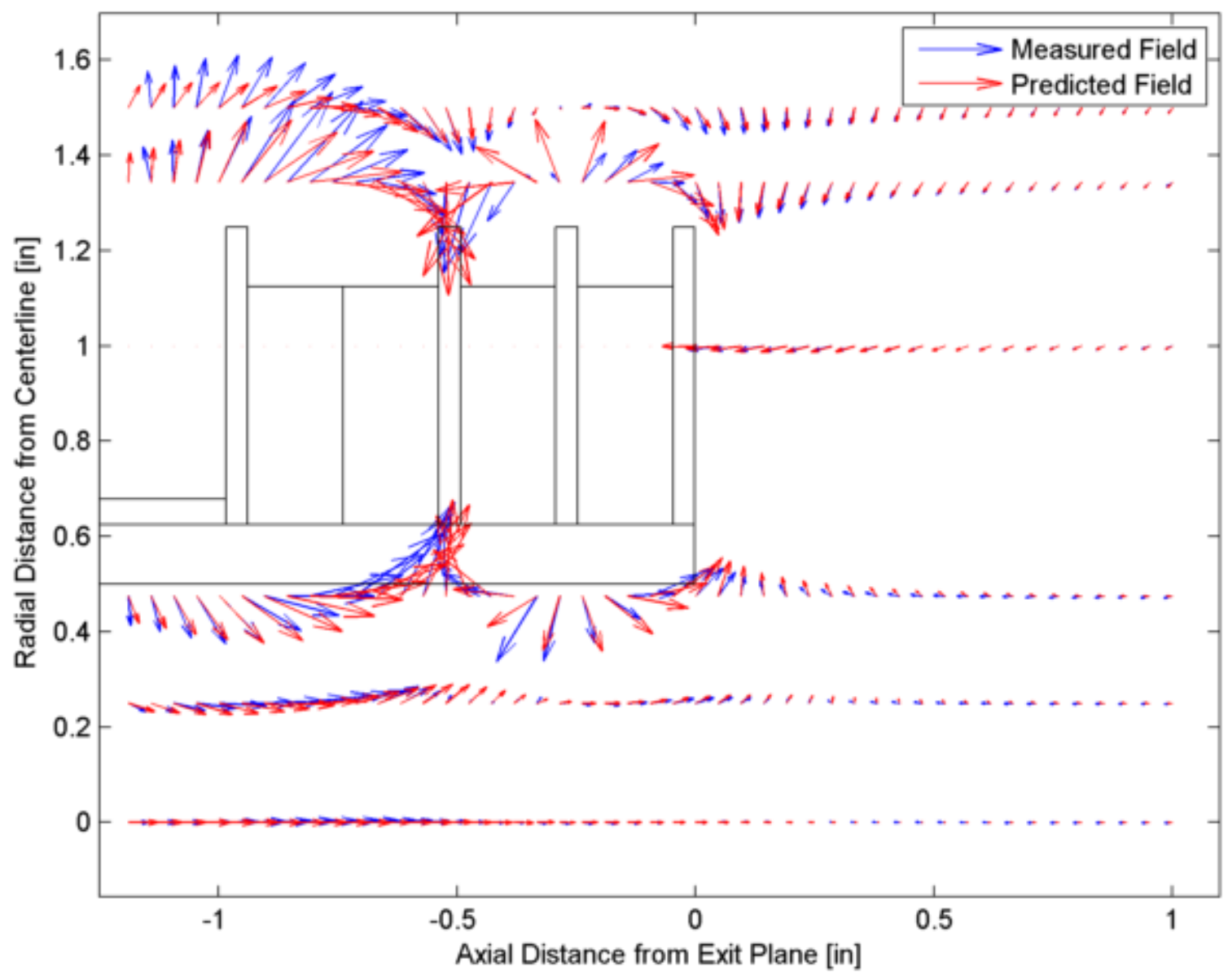

Figure 11. Empirical and Theoretical Arrow Surface Plot of HEMPT Magnetic Field. This magnetic field model was generated by taking many discrete measurements of the magnetic field of the HEMPT at a few y-values with an x step size of 0.048". Corresponding data from the COMSOL model was then overlaid.

Measurements were taken at $y$-values of $0.00,0.25,0.48,1.00,1.35$, and 1.50 inches, \pm 0.04 inches, from the centerline. For each of these $6 y$-values, measurements were collected at 47 evenly spaced $x$-values starting from 1 inch downstream of the thruster's exit plane. This resulted 
in an $x$ step size of $0.048 \pm .005$ inches, which was equivalent to 1 rotation of the threaded rod.

The $y$-values have a significant error value attached to them because the measurement stand used had some give in the y-axis, while the $\mathrm{x}$-axis was much more rigid.

From simply glancing at Figure 11, it appears that the empirical and theoretical data match reasonably well. However, the statistics summarized in Table 2 provide a different, more mixed conclusion.

Table 2. Magnetic Model Error.

\begin{tabular}{ll}
\hline Parameter & Value \\
\hline Field Angle Error & \\
Mean & $19.99^{\circ}$ \\
Median & $8.46^{\circ}$ \\
$I^{\text {st }}$ Quartile & $1.72^{\circ}$ \\
$3^{\text {rd }}$ Quartile & $25.36^{\circ}$ \\
Magnitude Error & \\
Mean & $141.11 \%$ \\
Median & $110.98 \%$ \\
$I^{\text {st }}$ Quartile & $65.82 \%$ \\
$3^{\text {nd }}$ Quartile & $150.56 \%$ \\
\hline
\end{tabular}

Based on these statistics, it seems that the COMSOL model is an adequate predictor of the angle in which the magnetic field will point at any given $x-y$ coordinate pair. The median error was $8.46^{\circ}$ and the data was heavily positively skewed such that $70 \%$ of measured values were within $15^{\circ}$ of predicted, and $93 \%$ of the measured values were within $45^{\circ}$. This is shown graphically in Figure 12. 


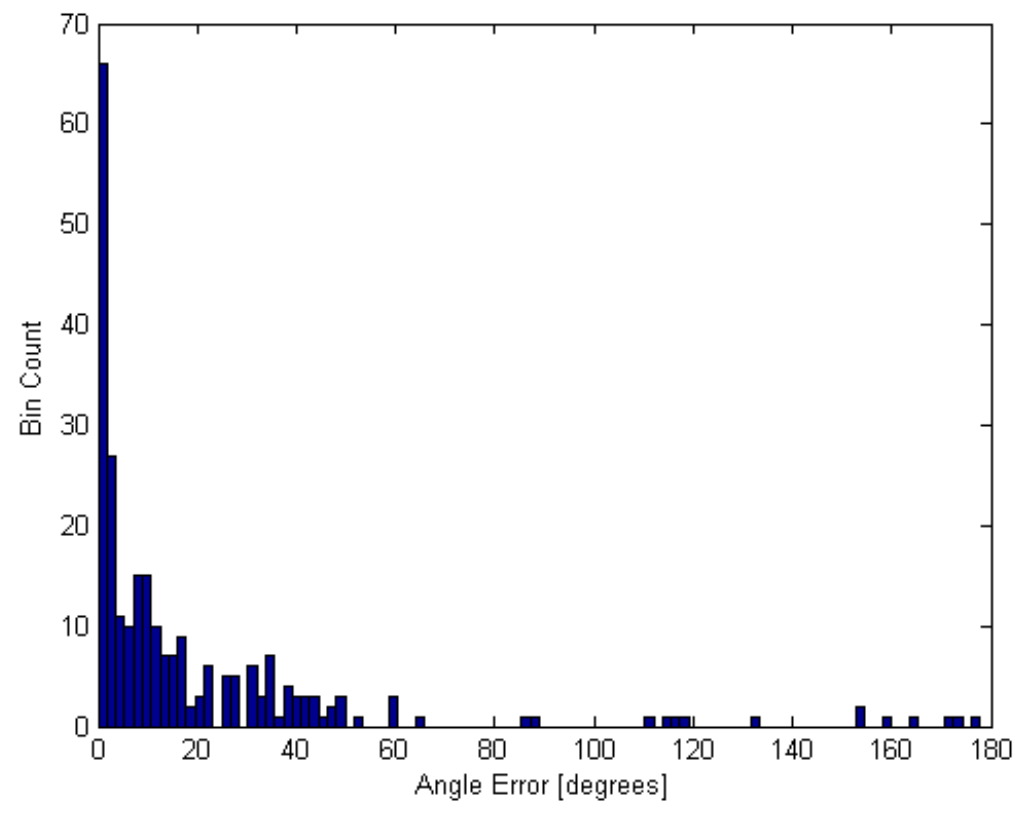

Figure 12. Histogram of Angle Error Between Measured and Predicted Field.

However, the COMSOL model is not a great predictor of the magnetic field magnitude.

This is particularly true for points near $(-0.3,1.35)$, just outside the thruster near the second (from the thruster's exit, looking in) iron plate. The predicted field is much stronger than what is acutally measured. This result can be explained by the fact that this is an example of a point in the model where the axial symmetry assumption breaks down. If the thruster were truly axially symetric, the rings of rectangular magnets would instead be solid anulluses. Because this is not the case, the outside of the rings has gaps. The 2-D slice through which data was measured to form this plot happened to pass through one of these gaps in the rings at this point, resulting in a large decrease in magnetic field strength.

Notice that within the thruster the measured magnitude of the magnetic field (as well as its direction) matches much more closely to what the model predicts. This is because the edges of the 10 magnets in each ring are touching each other to form a nearly perfect decagon with sides of length 0.4 ", which is a fairly good approximation for a circle. In essence, the assumption of axial symmetry is very close to true within the thruster, but not outside of it. 
Another potential cause of poor prediction capabilities was the modelled value for the strength of each individual magnet. All magnets were given a surface magnetization of $300 \mathrm{mT}$ in COMSOL. The actual magnets tended to have a field strength within $\pm 15 \mathrm{mT}$ from this value. Any deviation from the assumed value of $300 \mathrm{mT}$ could cause some level of deviation from the model's prediction. Further, as mentioned previously in Table 1, the actual magnetic properties of the materials were probably different in some ways from what was modelled.

A much more likely cause of poor magnetic field magnitude prediciton capability is the significant uncertainty in the $y$-values. As mentioned previously, the $y$-axis of the measurement stand had some give to it. The HEMPT is a relatively small thruster with densely packed, strong magnets, so the gradient of the magnetic field is often quite steep. Even a small departure in the $y$ direction can result in a large difference in both magnitude and direction from what the model predicts. This histogram of the magnitude error is shown in Figure 13.

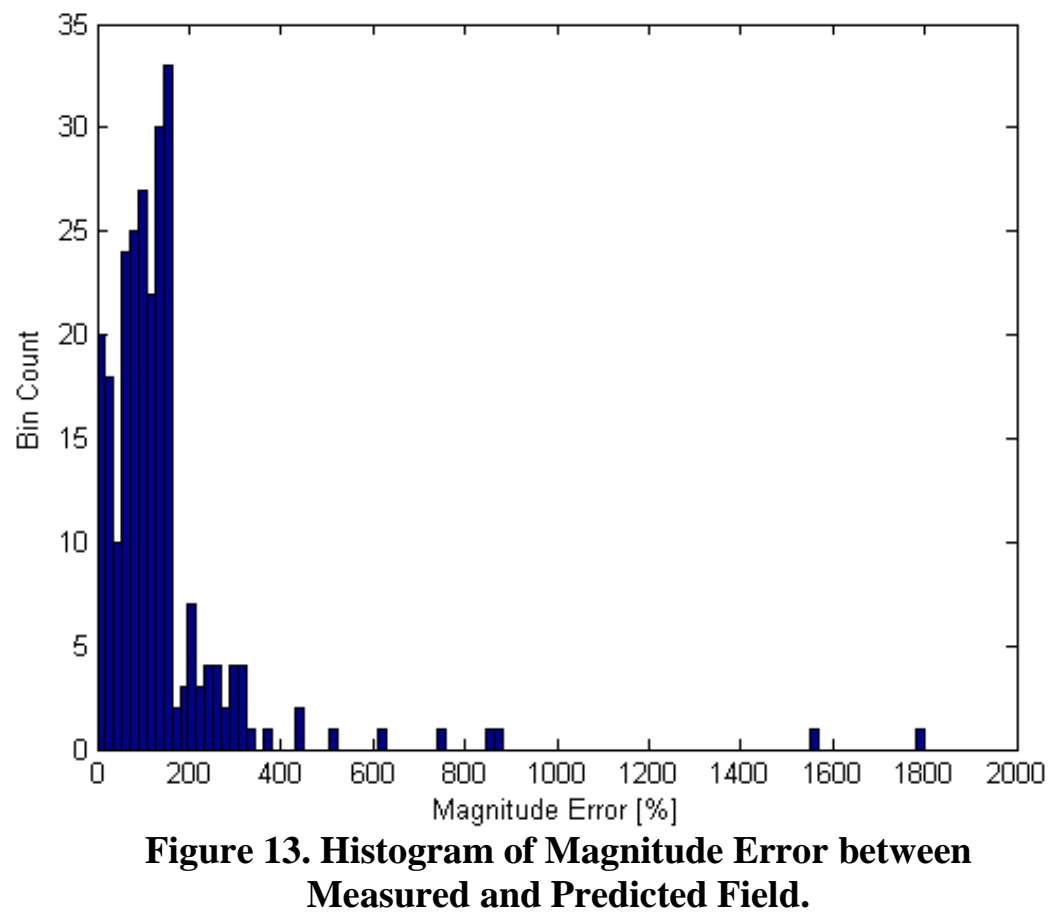

Measuring the magnetic field has shown that the model isn't perfect. Luckily, the magnitude of the magnetic field is not a metric which is extremely relevant for this thesis. The focus, rather, is on the change in field magnitude and field direction when a magnetic shield is 
added to the thruster. For this reason, the presented model was deemed sufficient. Further qualitative evidence of the validity of the magnetic field model is provided in Figure 14. Here, the magnetic field model is overlayed on a side view image of the actual HEMPT in operation. From this image it is clear that the plasma plume tends to follow the model's magnetic field lines very closely.

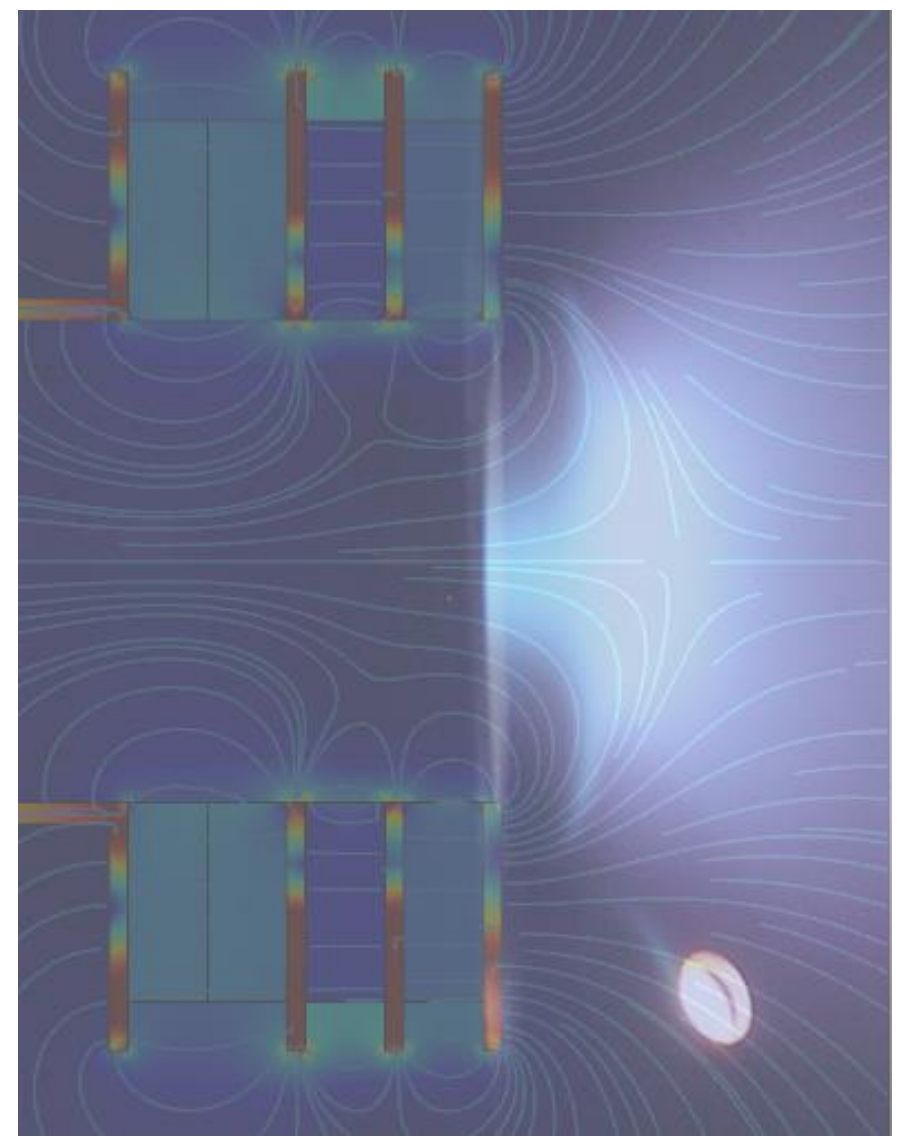

\subsection{SHIELD DESIGN}

Figure 14. Overlay of COMSOL Model and actual thruster in operation.

After forming an initial model of the HEMPT's magnetic field, the next step was to use this model to design magnetic shields to improve the thruster's performance by reducing the divergence of its plume. Electrically charged particles, such as ions and electrons, tend to follow magnetic field lines. When an electrically charged particle encounters a field line, it experiences a force which causes the particle to spiral around the field line. However, the particles also have an initial velocity with some portion of that velocity along the field line. The net effect is that electrons and ions tend to move along magnetic field lines while simultaneously spiraling around them.

Revisiting Figure 7, one can imagine an ion in the ionization chamber near the anode and near the thruster centerline. The path the ion takes to exit the thruster is determined by its initial velocity and starting position. Some of the streamlines near the exit of the thruster are strongly radial, which means an ion following these streamlines will have a tendency to pull away from 
the centerline. This tendency to diverge from the centerline is the main contributor to the hollow shape of the thruster's plume. This radial component of the magnetic field is what any implemented shield should reduce and is the first measure of a shield's theoretical performance.

Cast-iron was used for creating the shields. This same material was used by the PPPL to mitigate a hollow plume in a CHT [11]. Also, it was provided by Dr. Goebel for Scott's thesis and some material was left over. The main benefit of cast-iron for use as a magnetic shield is that it has high relative permeability, meaning it will have substantial effects on magnetic fields.

Secondly, it is relatively easy to machine.

\subsection{INITIAL SHIELD (SHIELD 2)}

The first shield design studied was one Scott McGrail recommended in his thesis. It is a $0.2^{\prime \prime}$ thick shield with a $45^{\circ}$ chamfer. The shield covers the exit face of the thruster and turns $90^{\circ}$ to cover much of the outside of the thruster as well. Scott McGrail originally considered 6 different shield designs. His research and modeling showed that this shield, his second shield design, was most promising for thruster performance improvement. This shield will be referred to as Shield 2, the same name he gave it, throughout this thesis. It is shown in Figure 15. Note Shield 1 will not be discussed here, so Shield 2 refers to the first shield examined in this paper. 


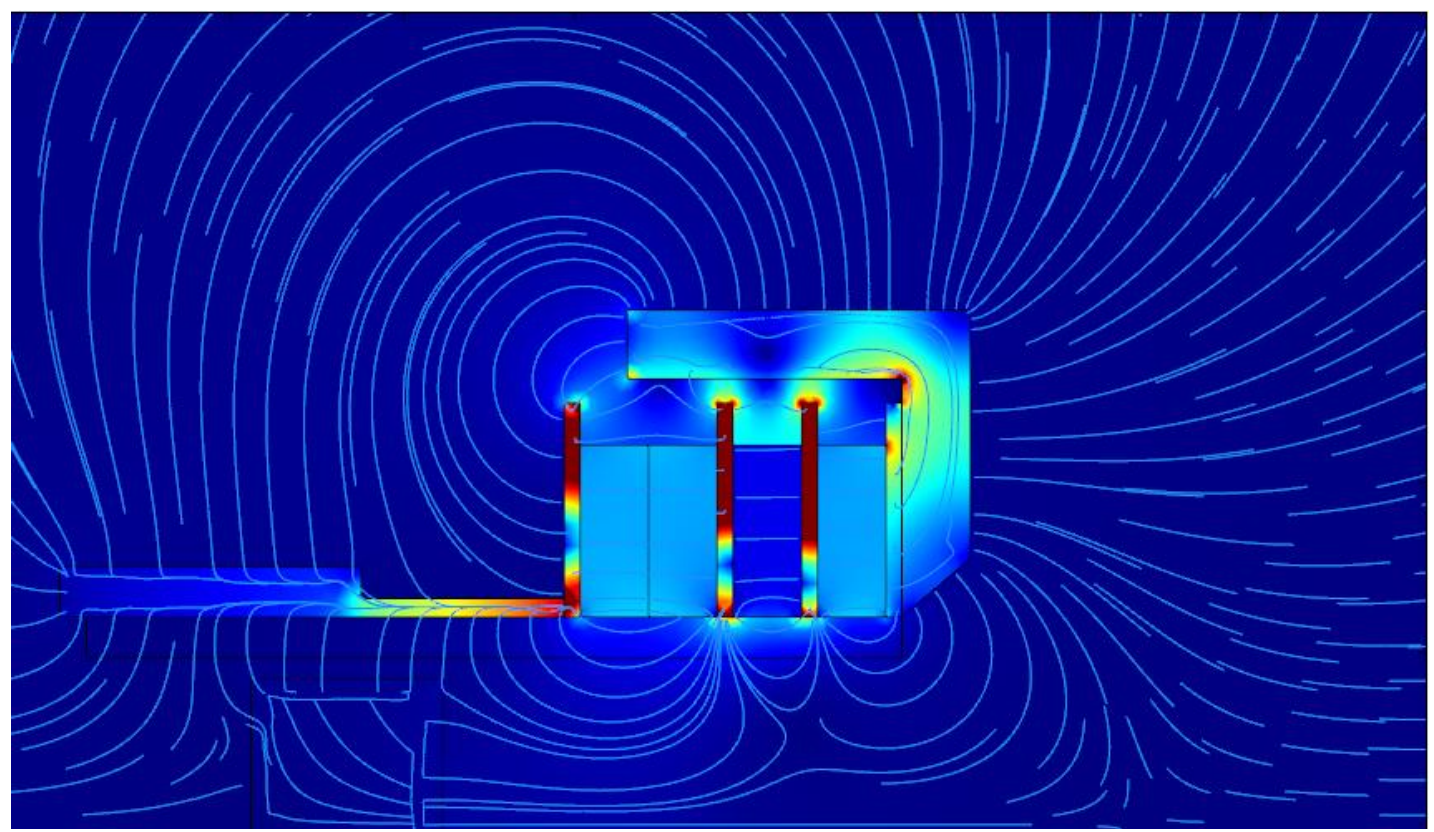

Figure 15. Scott's Shield 2 Modelled in COMSOL. The shield is 0.2 " thick throughout, with a $45^{\circ}$ chamfer at the thruster exit.

From visual inspection of Figure 15, it is difficult to discern the effect that the shield has on the field lines, and especially on the strength of the field. To more easily compare the effects of various shields on the thruster's magnetic field, the radial components of the field were plotted (at various y-locations) against the x-location at which they are predicted to occur. It is useful to compare the shield's effects versus the baseline configuration with no shield. This comparison is shown in Figure 16. 


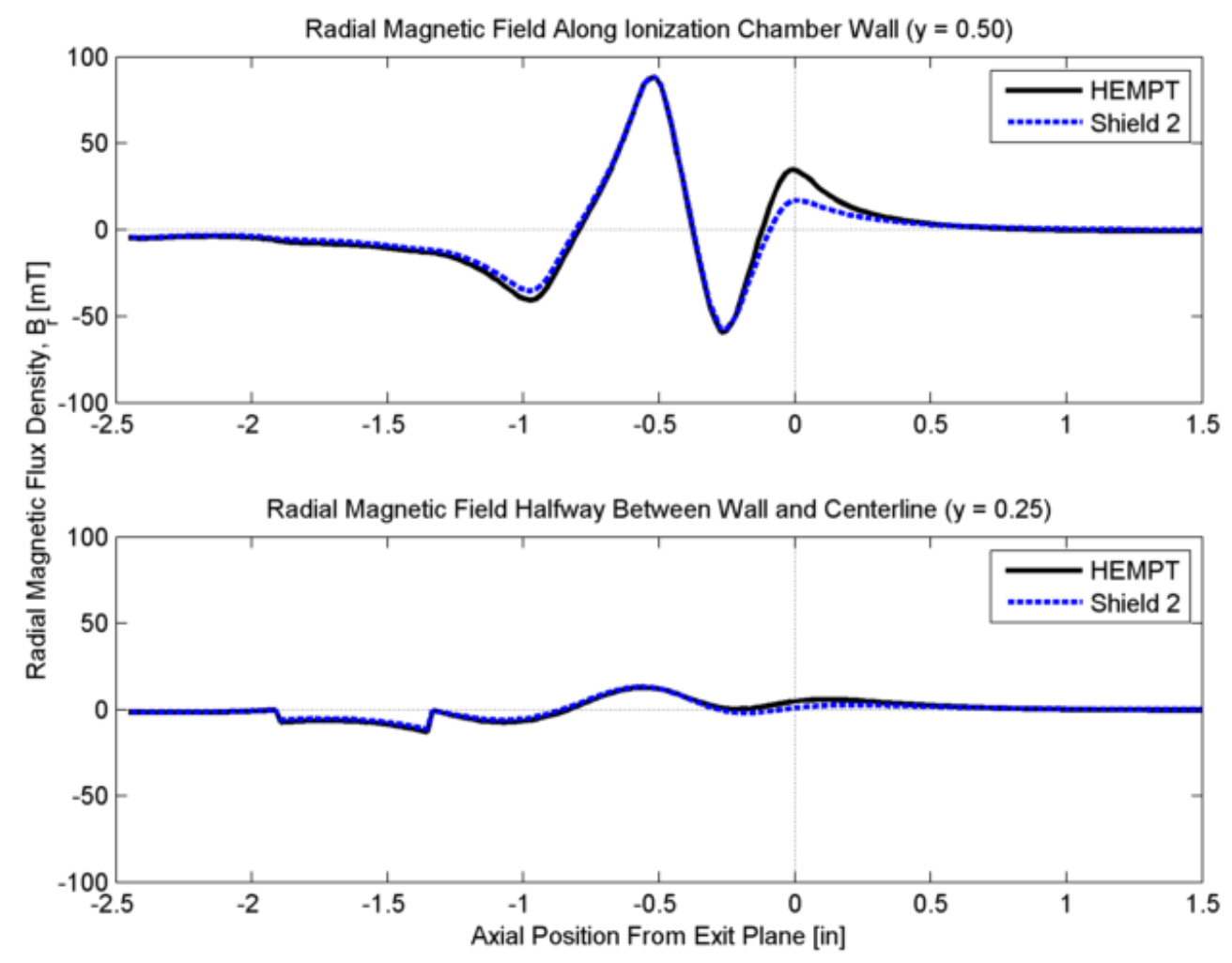

Figure 16. Radial Magnetic Field at the Ionization Chamber Wall and Halfway From Centerline to Wall for Shield 2.

The radial magnetic flux density was plotted at these two locations because they are both representative samples in their own way: The ionization chamber wall shows the closest that the ions will get to the periodic permanent magnets just behind the chamber wall, while the halfway point between the centerline and wall shows a more moderate field that the ions may experience. The radial field at the centerline of the thruster was not plotted because the model predicts that the field will be $100 \%$ axial at this location. The only way a chosen shield would change that is if it had some type of axial asymmetry. All shields considered in this thesis are axially symmetric.

As Figure 16 shows, shield 2 has little to no effect on the field at the majority of xlocations. The two places where there is a noticeable change are the exit plane and around -1" from the exit plane, inside of the thruster. This location at -1 " corresponds to the most upstream location of the periodic permanent magnet assembly. Essentially, the shield affects the thruster's magnetic field at both ends of the permanent magnet assembly. Further, in both cases, the affect 
seems to be desirable. The radial component of the magnetic field is reduced at both locations. The most significant effects of the shield are seen along the ionization chamber wall. At the -1 " (or, more precisely, $-0.974 ")$ location the maximum radial field strength reduction is from -40.89 $\mathrm{mT}$ (unshielded) to $-35.29 \mathrm{mT}$, a $13.70 \%$ reduction. At the exit plane where the radial magnetic field is more of a concern, a much more significant reduction is observed. The unshielded HEMPT has a magnetic field strength of $35.35 \mathrm{mT}$ while the shielded HEMPT has a magnetic field strength of $15.15 \mathrm{mT}$. This represents a $57.14 \%$ reduction. These results are summarized in Table 3 .

Table 3. Radial Magnetic Field Strength, $B_{r}$, at X-Locations at Ionization Chamber Wall.

\begin{tabular}{llll}
\hline X-Location [in] & Unshielded $\mathbf{B}_{\mathbf{r}}[\mathbf{m T}]$ & Shield 2 $\mathbf{B}_{\mathbf{r}}[\mathbf{m T}]$ & Percent Reduction \\
\hline 0.000 & 35.35 & 15.15 & $57.14 \%$ \\
-.974 & -40.89 & -35.29 & $13.70 \%$ \\
\hline
\end{tabular}

From these results alone it appears that Shield 2 causes very beneficial alterations to the baseline magnetic field. However, the shield does more than modify the radial component of the magnetic field: it also alters the axial component. Inside the thruster, the net effect of the addition of the shield is to reduce the strength of the field. This is clearly evident in Figure 17.

This result is a logical one. It makes sense that the magnetic field strength inside of the thruster would decrease when a mass of iron is placed just outside of the thruster. The iron can be thought of as a path of less resistance for the magnetic field lines. It has much higher relative permeability than the vacuum of the ionization chamber. The result is that the field strength outside of the thruster increases, causing the internal field to decrease. 


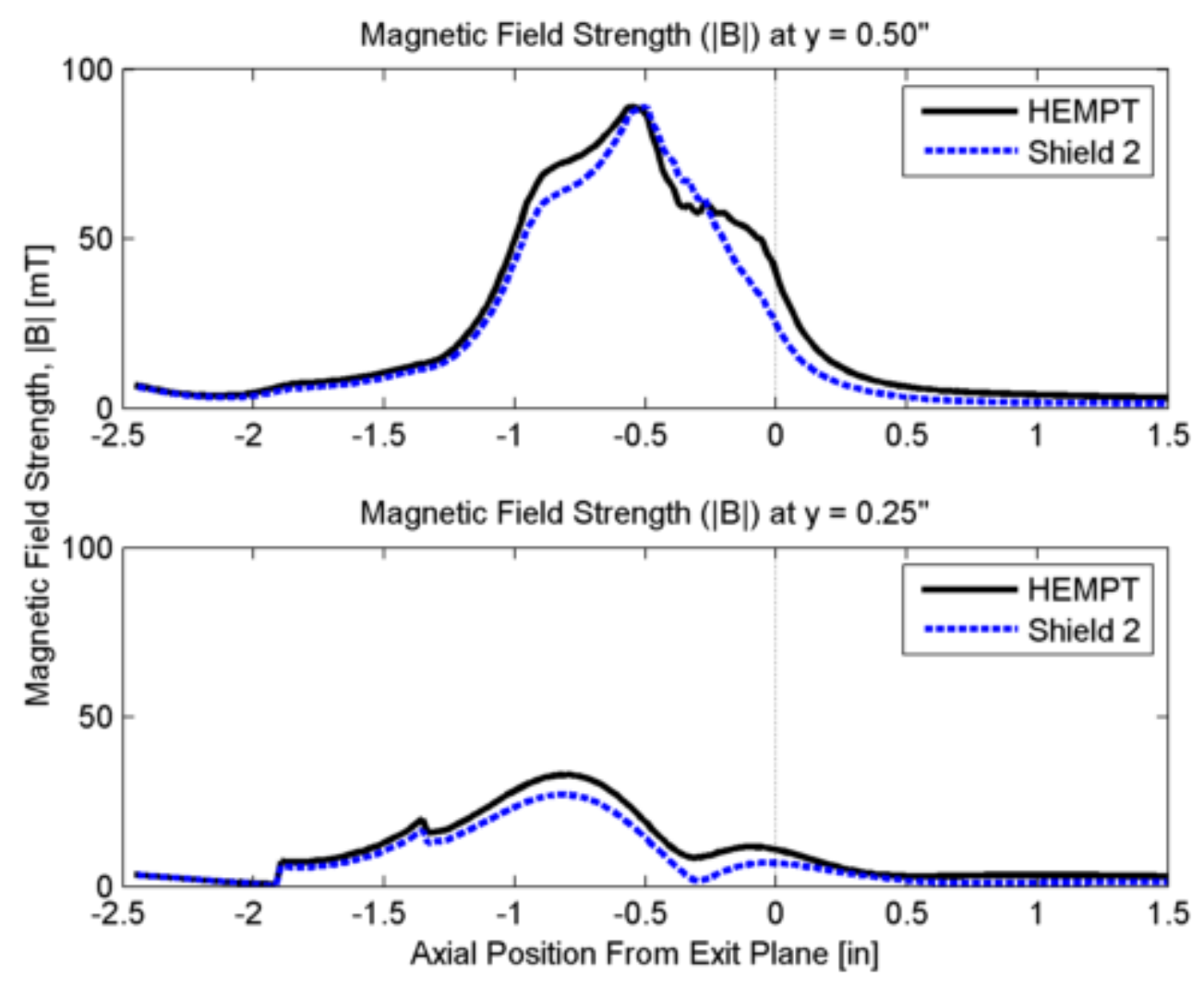

Figure 17. Magnetic Field Strength $(|\vec{B}|)$ at Ionization Chamber Wall and
Halfway from Centerline to Wall for Shield 2 .

The internal magnetic field of the thruster is designed to efficiently impede electrons

from moving upstream toward the anode. Because of this, a widespread and significant decrease in field strength within the thruster could be very detrimental to its performance. Whether or not the decrease in field strength in Figure 17 is large enough to significantly affect thruster performance is not known. This shield was previously tested with somewhat inconclusive results due mostly to measuring apparatus issues.

\subsection{SUGGESTED SHIELD (SHIELD 7)}

Scott McGrail suggested another shield design which was never tested due to time constraints. This new shield, which will be referred to as Shield 7, was similar to Shield 2 except there was no $90^{\circ}$ turn: The shield only covered the exit face of the thruster. His theory was that this would be a good compromise design. Theoretically, it should decrease the radial magnetic 
field component near the thruster's exit plane without significantly affecting the magnetic field strength throughout the thruster. Indeed, this is what the COMSOL model suggests as well, which is evidenced in Figure 18 and Figure 19.

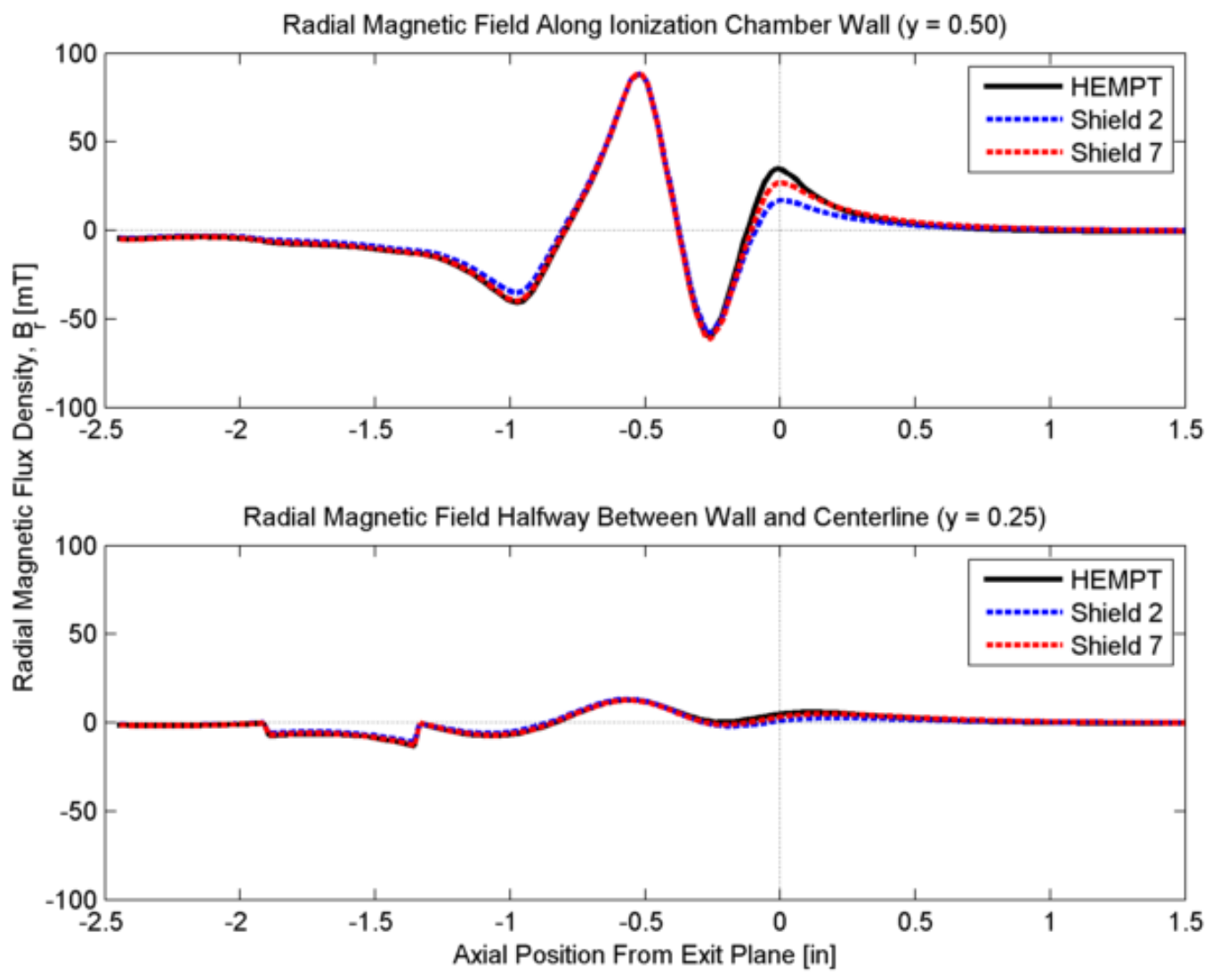

Figure 18. Radial Magnetic Field at Ionization Chamber Wall and Halfway from Centerline to Wall with Shield 2 and Shield 7. 


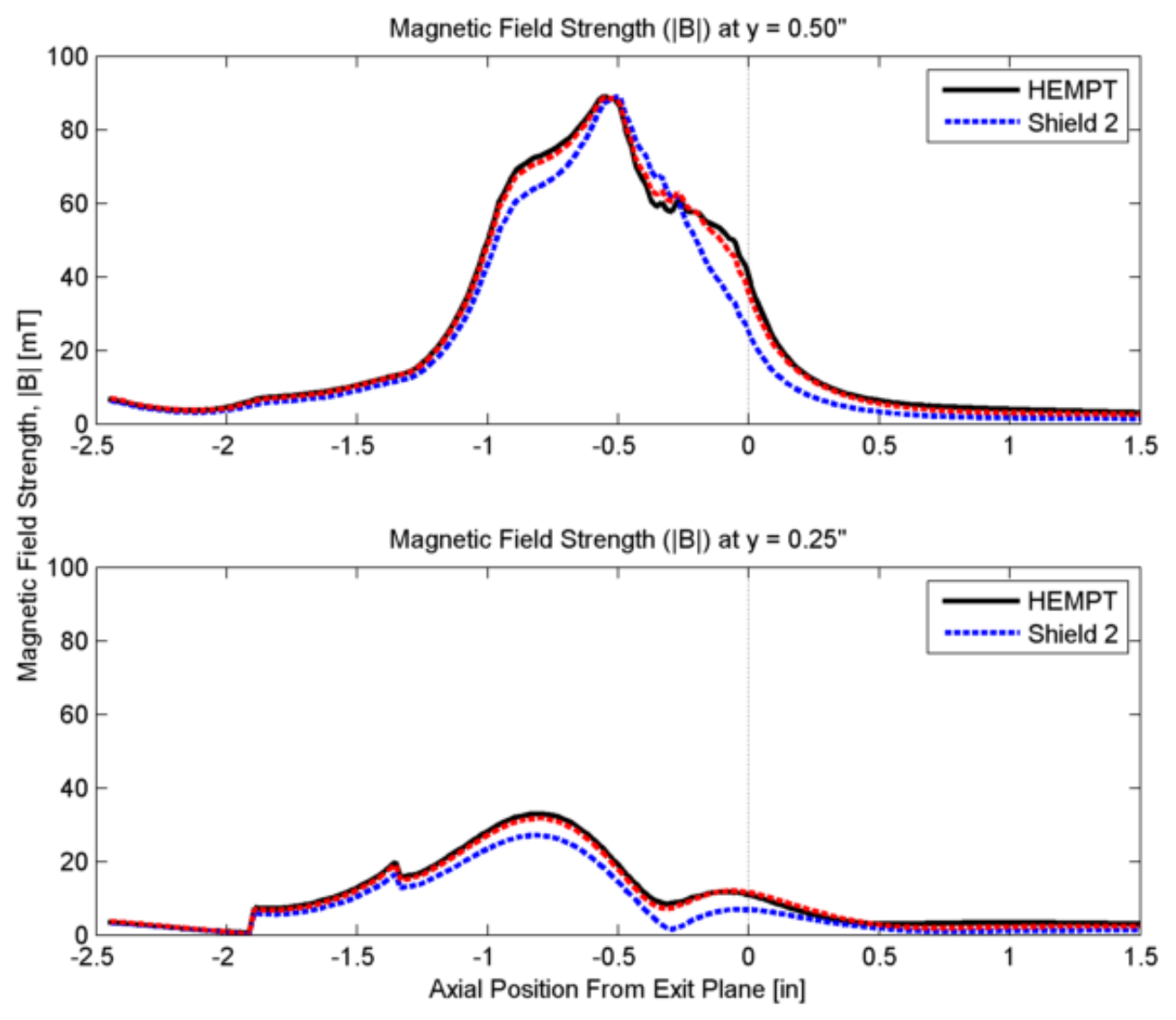

Figure 19. Magnetic Field Strength $(|\vec{B}|)$ at Ionization Chamber Wall and Halfway from Centerline to Wall for Shield 2 and Shield 7.

Figure 18 shows that Shield 7 still forces a decrease in the radial magnetic field at the thruster's exit, though the change is admittedly less pronounced than with Shield 2. Figure 19 shows that Shield 7 has barely any effect on the overall strength of the magnetic field. Both at the wall and halfway to the wall, the magnetic field strength with and without the shield are almost the same. Theoretically, this is a much more useful shield design than Shield 2.

\subsection{NEW SHIELD DESIGNS}

Further shield designs were modeled and analyzed. Each design is shown in Figure 20. These shields were created by varying some part of the geometry of Shield 2 to see if any improvements could be made. Shield parameters were not optimized, however, a task which is 
outside of the scope of this thesis. The goal here was only to find potential improvements upon Shield 2 and Shield 7.
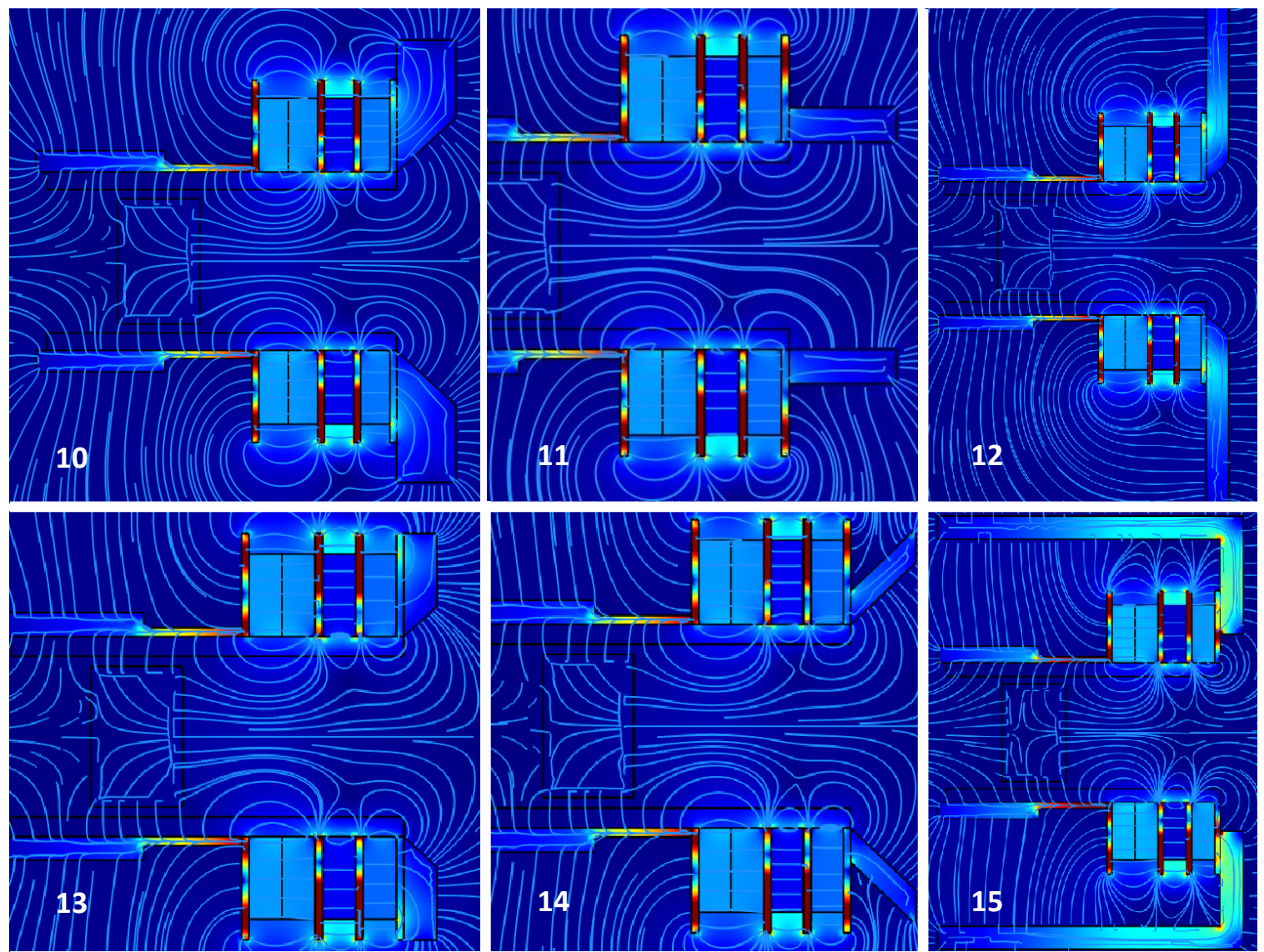

Figure 20. Magnetic Shields Designed in COMSOL for Possible Testing. Shield 10, 11, 12, 13, 14, and 15 from top left to bottom right.

Shield 10 is twice as thick (0.4") as Shield 7, while Shield 11 is extended out in the direction of the thruster centerline. Shield 12 is a longer version of Shield 7 at twice the length. Shield 13 is slightly shorter than Shield 7 such that the edge of the shield is flush with the edge of thruster. Shield 14 is 0.2 " thick but the entire shield extends off at a $45^{\circ}$ angle. Finally, Shield 15 was modelled based on the research from Raitses et al. regarding magnetic shielding of CHTs. Though their research findings are not exactly applicable to the HEMPT, the effects of this shield were still studied. Plots of the magnetic fields of these shield designs are shown in Figure 21. 

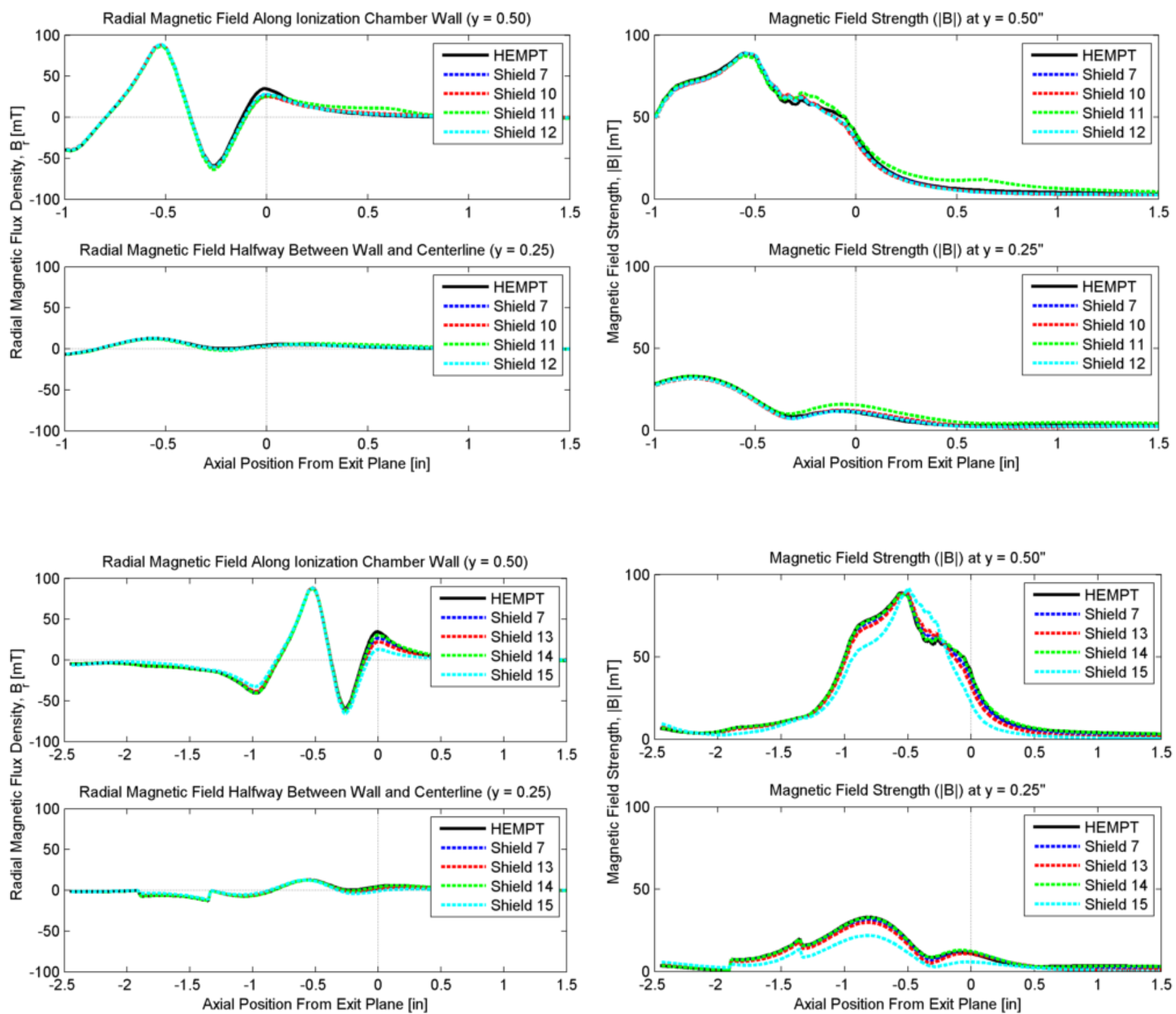

Figure 21. Radial Magnetic Field and Magnetic Field Strength for Various New Shield Designs Along the Thruster's Ionization Chamber Wall and Halfway from the Wall to the Centerline. The X-Axis for Shields 10-12 was shortened slightly to increase data clarity. 
The performance of each shield is summarized in Table 4, and a more detailed discussion is provided below.

Table 4. Summary of Modeled Magnetic Shield Performance.

\begin{tabular}{|c|c|c|}
\hline $\begin{array}{l}\text { Shield } \\
\text { Number }\end{array}$ & Description & Conclusion \\
\hline Shield 7 & Baseline & Good Test Candidate \\
\hline Shield 10 & 2x thickness of Shield 7 & Very similar to Shield 7 \\
\hline Shield 11 & $\begin{array}{l}\text { Extended in centerline } \\
\text { direction }\end{array}$ & Raises strength inside chamber \\
\hline Shield 12 & Longer version of Shield 7 & Very similar to Shield 7 \\
\hline Shield 13 & Shorter version of Shield 7 & $\begin{array}{l}\text { Better radial field reduction than Shield } 7 \text {, larger } \\
\text { change inside chamber. }\end{array}$ \\
\hline Shield 14 & $45^{\circ}$ angle & Only small change to radial field at exit. \\
\hline Shield 15 & Long, wrapped around & Large change to internal field. \\
\hline
\end{tabular}

Shield 10 behaves very similarly to Shield 7, making it difficult to discern the difference between the two in the plots. This makes sense since Shield 10 is simply a thicker version of Shield 7 . This means that Shield 7 is still the better choice because it will weigh roughly half of what Shield 10 will. Shield 11 has a tendency to raise the field strength within the thruster in many cases, while simultaneously increasing the radial component at the exit. Clearly, this rise in radial magnetic field strength of Shield 11 is no good. Shield 12, like Shield 10, follows the behavior of Shield 7 very closely. With this is mind, Shield 7 is once again the better choice because it uses less material. Shield 13 is also similar to Shield 7. It reduces the radial magnetic field at the exit by a little more than Shield 7 (36.63\% vs. $24.21 \%$ reduction) but also causes more significant shield strength changes throughout the thruster than Shield 7. This makes Shield 13 a strong contender, but Shield 7 seems like a more well-rounded pick. Shield 14 causes only a small reduction to the radial magnetic field at the exit, with little effect on the radial or total magnetic field strength at other points in the thruster. This is similar behavior to Shield 7, but with fewer benefits. Finally, there's Shield 15, which was based on research into magnetic shielding for cylindrical Hall thrusters [11]. Shield 15 does provide a large drop in radial magnetic field magnitude at the thruster's exit. However, this comes at the cost of a large reduction in the internal field of the 
thruster. Much like Shield 2, this shield creates a path of less resistance for the magnetic field outside of the thruster.

From the designs examined by Scott McGrail and the new ones presented in this thesis, Shield 7 seems to be the most promising candidate, so it was manufactured and tested. 


\section{PLASMA DIAGNOSTICS PROBES}

Two probes were used for data collection during testing: A retarding potential analyzer (RPA) and a Faraday probe. The RPA is a gridded electrostatic device which filters ions from the plasma based on their kinetic energies which allows for the determination of the plume's ion energy distribution [12]. The Faraday probe is a much simpler device. It is an electrostatic probe comprised of an exposed electrode which is capable of measuring the thruster's beam current density [13]. Both of these probes will be explored further in the following section.

\subsection{RETARDING POTENTIAL ANALYZER (RPA)}

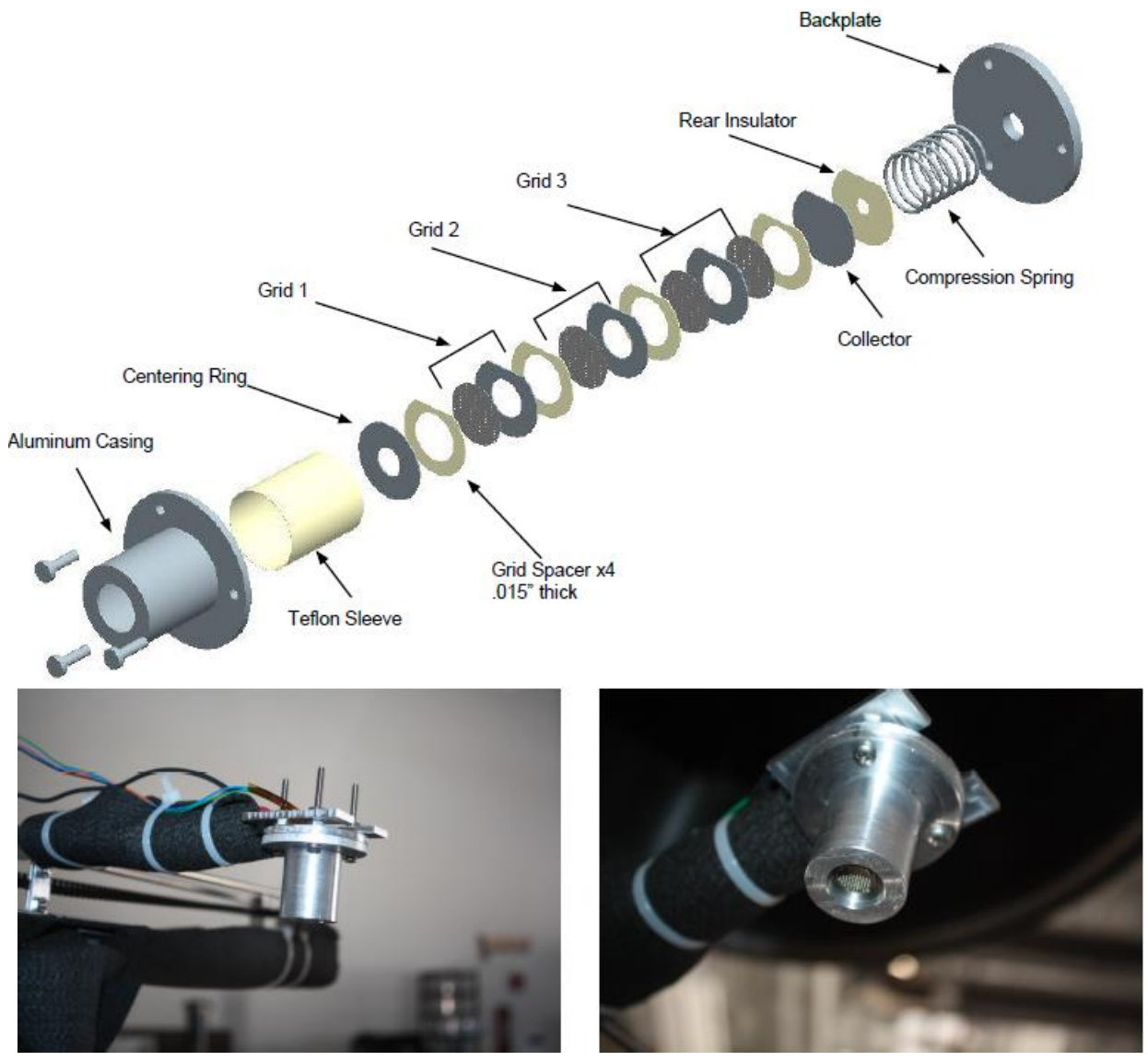

Figure 22. Exploded View of the RPA CREO Model and 2 Images of the Actual RPA Used for Testing. These images courtesy of Scott McGrail [15], reproduced here with permission. 
The RPA used for testing in this thesis is a 3-grid variety in an aluminum casing. Each grid is biased independently. Grid 1, the first grid seen by any potentially collected plasma (see Figure 22), is at the floating potential. The second grid is biased at a relatively low negative voltage, around $-20 \mathrm{~V}$. This grid is responsible for repelling electrons, ensuring the collector is only seeing ions. Grid 3 is called the discriminator or retarding grid. Grid 3 is swept from zero volts and up, until current is no longer collected. Ions which encounter the discriminator grid must have high enough kinetic energy in order to pass it at a given voltage. This means that as the voltage of grid 3 is increased the current seen by the collector decreases. This effect can be seen in the sample RPA plot in Figure 23. The generated current is read by a DAQ system. The probe is moved through several different points in the plume and a voltage sweep performed at each point.
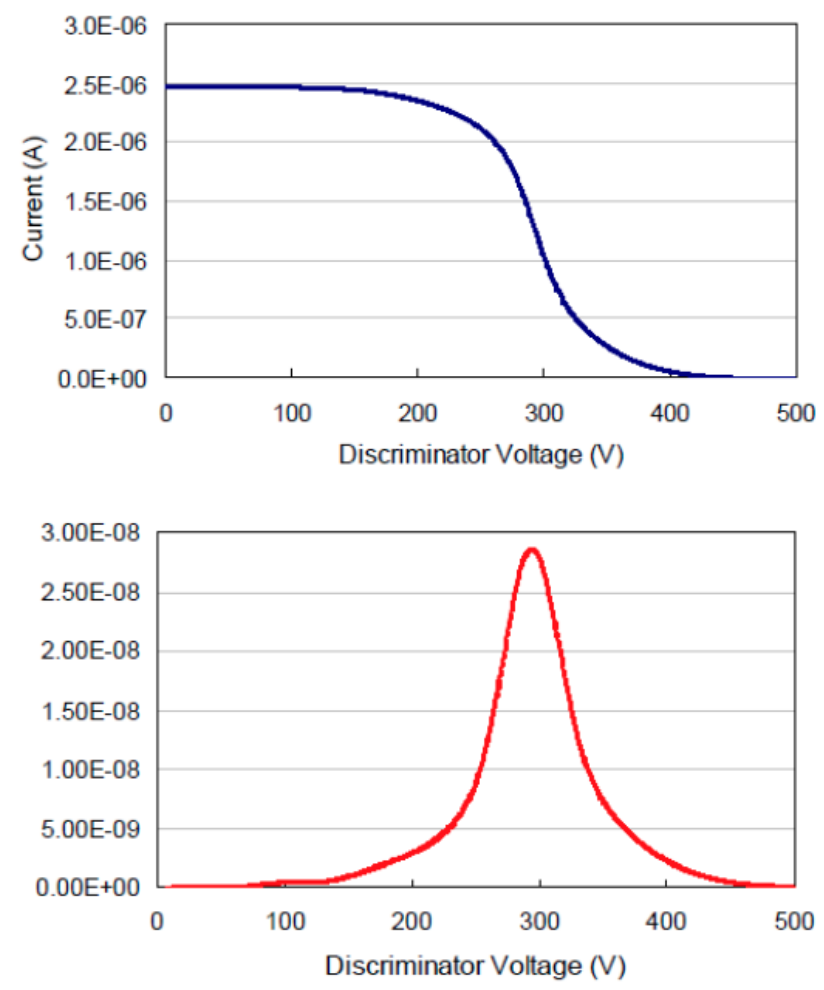

Figure 23. Example of RPA Curve and its Derivative [23].

The RPA's grids are electrically isolated from each other using 0.015 ” thick Teflon sheeting, cut into small rings. A Teflon sleeve isolates the grids from the probe's aluminum 
casing. A notch was cut out of the top of the Teflon rings to allow Teflon-coated copper wires to be fed through the probe to the various grids. The wires were spot-welded to the grids and the whole assembly was compressed with a spring and backplate. The grid dimensions and resultant transparencies are summarized in Table 5

Table 5. RPA Grid Dimensions.

\begin{tabular}{llll}
\hline Component & Purpose & Mesh Size $(\mu \mathrm{m})$ & Grid Transparency \\
\hline Grid 1 & Plasma & 175 & 0.391 \\
Grid 2 & Electron Repelling & 40 & 0.191 \\
Grid 3 & Ion Discriminator & 60 & 0.490 \\
\hline
\end{tabular}

The RPA was built and used for previous HEMPT testing. However, it had a fairly major flaw. The RPA was incapable of producing ion energy distribution plots because even at high retarding potentials $(600 \mathrm{~V})$, the RPA was still recording significant currents. Instead, the RPA was used as a glorified Faraday probe to collect ion current at several points in the plume in order to create the ion beam profile. To remedy this problem, the RPA was disassembled for this thesis. A possible electrical short to ground was identified within the RPA. This was fixed and the RPA was reassembled for use in testing.

\subsection{FARADAY PROBE}

The RPA is a relatively complex, multi-gridded plasma diagnostics tool. In contrast, a Faraday probe is very simple. For this thesis, a JPL-style nude Faraday probe was used. A schematic of this type of probe, as well as an image of the one used in this thesis, is shown in Figure 24. 

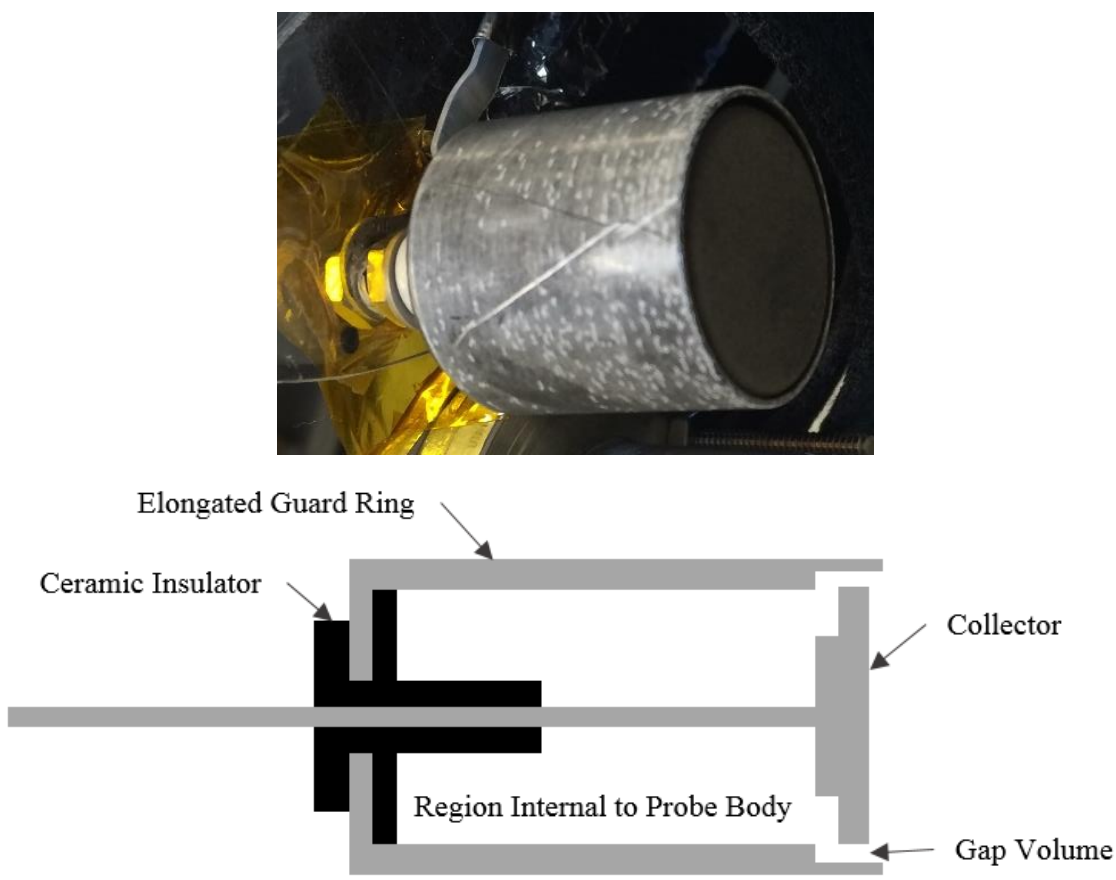

Figure 24. Image of JPL-style Nude Faraday Probe and Schematic [13].

Faraday probes are used to measure ion current density distribution of plasma. The probe is biased below plasma potential, at around $-20 \mathrm{~V}$. This ensures that the vast majority of plasma electrons are repelled by the probe and ions are drawn to it. When an ion impacts the collector face a small current is produced and read by a DAQ system connected to the probe. Many ions impact the collector at once, producing a current which can then be divided by the collector's area to get a value for beam current density. The collection face is aluminum which has been spraycoated with tungsten to minimize secondary electron emission [14].

The guard ring is an essential component of the Faraday probe which helps ensure that the current density measurement is accurate. Without the guard ring, if an ion is approaching the probe in a fully axial direction in such a way that it would just miss the collector face, it is possible that this ion's path would bend to hit the collector. This skews the current density measurement because the collector, in effect, has a larger area than what is measured and predicted with simple geometry. By including the guard ring and biasing it at the same potential as the collector, those ions would continue through the gap between the collector and guard ring 
or be collected by the guard ring, where they aren't measured. This means they would not produce current on the collector.

Nominal dimensions for the JPL nude Faraday probe are summarized in Table 6.

Table 6. Faraday Probe Nominal Dimensions [14].

\begin{tabular}{ll}
\hline Component & Dimension $(\mathrm{cm})$ \\
\hline Collector Diameter & 2.31 \\
Gap Thickness & 0.23 \\
Guard Ring Outer Diameter & 2.540 \\
Guard Ring Thickness & 0.074 \\
\hline
\end{tabular}




\section{PROBE DATA ANALYSIS}

The major, overarching goal of this research is to obtain data on the HEMPT's performance with and without a magnetic shield. The main performance metrics for any spacecraft propulsion system are thrust produced, specific impulse, and overall efficiency. The HEMPT is no different in this regard. Thrust will be derived (not directly measured) from the power of the beam, the ion species in the beam, and the beam divergence angle. Specific impulse is then easily derived from thrust and the rate of argon gas consumption.

\subsection{RPA DATA ANALYSIS}

Current is collected by the RPA during a voltage sweep from $0 \mathrm{~V}$ to high positive voltage, until collected current is zero. Because the first derivative of the collected ion curve during a voltage sweep is proportional to the ion energy distribution, the ion voltage distribution function, $f(V)$, can be found using the relationship,

$$
\frac{d I_{\text {probe }}}{d V_{\text {probe }}}=-\frac{Z_{i}^{2} e^{2} n_{i} A_{e}}{M} f(V)
$$

where $I_{\text {probe }}$ is the current collected by the RPA, $V_{\text {probe }}$ is the voltage of the RPA's discriminator grid, $Z_{i}$ is the charge state of the ionized gas, $e$ is the fundamental charge, $n_{i}$ is the number of ions, $A_{e}$ is the effective area of the probe, and $M$ is the mass of a single ion of propellant, in this case Argon.

For this relationship to work, the assumption that all of the ions in the plume are singly charged must be made. This is false, as plasma thrusters tend to have a significant amount of doubly (and some triply) charged ions. This is why this relationship provides the ion voltage distribution function instead of the ion energy distribution function [15].

Because of this, the RPA's measurements provide only an estimate for the beam voltage, $V_{b}$. This is achieved by forming the collected ion curve and taking its derivative. The most 
probable value for $V_{b}$ is then given by the voltage of the largest peak. It is only the most probable because the exact beam voltage is given by

$$
V_{b}=V_{R P A}-\phi_{p}
$$

where $V_{R P A}$ is the most probable beam voltage, $\phi_{p}$ is the plasma potential of the beam, and $V_{b}$ is the actual beam voltage. In order to get the actual plasma potential a Langmuir probe and another power supply (as well as another DAQ channel) would be needed. Because of this, the most probable beam voltage, $V_{R P A}$ was planned to be used as the actual beam voltage, $V_{b}$. However, due to problems with the RPA, described later, this was not possible and a further assumption had to be made.

\subsection{FARADAY PROBE DATA ANALYSIS}

The Faraday probe collects ion current at each pre-determined point in the plume. This is useful for building a profile of collected ion current as a function of radial distance from the thruster's centerline, which can then be integrated to get the total beam current,

$$
I_{b}=2 \pi \int_{0}^{r} j_{b}(r) \cdot r d r
$$

where $j_{b}(r)$ is the collected current density in $\frac{A}{m^{2}}$ as a function of radial distance from the centerline in meters and $I_{b}$ is the total beam current expressed in Amps. However, the raw collected current isn't sufficient to accurately calculate the total beam current because there are a number of factors which must first be accounted for, such as charge exchange and transparency.

Prior to reaching the Faraday probe, ions from the thruster may interact with neutrals in the chamber and exchange charge. This interaction is generally one in which the neutral gives up an electron to the ion without an exchange in momentum. This results in a fast-moving neutral with the original ion's velocity and a slow moving ion with a random velocity [14]. This results in a lower ion current seen by the Faraday probe because many of the ions ejected by the thruster 
become neutral prior to reaching the probe. A CEX correction factor is used to account for this, and is given by

$$
\left(\frac{j}{j_{0}}\right)_{A r^{+}}=e^{-n_{0} \sigma_{1} z}
$$

where $\mathrm{n}_{0}$ is the neutral density in the chamber $\left(\frac{\text { particles }}{\mathrm{m}^{3}}\right), \mathrm{z}$ is the distance from the thruster's exit plane to the probe in meters, and $\sigma_{1}$ is the effective cross sectional area of the charged particle in square meters. $\sigma_{1}$ is calculated using the equation,

$$
\sigma_{1}=\frac{1}{2}[18.96-0.83 \ln (\mathrm{v})]^{2}
$$

where $\mathrm{v}$ is the velocity of the ions expressed in $\mathrm{cm} / \mathrm{s}$, which can be obtained from RPA measurements. Equation 14 holds true for Ar-Ar+ charge exchange interactions [16]. The value produced by this equation must be converted from $\AA^{2}$ to $\mathrm{m}^{2}$. As will be explained in later sections, the RPA ion energy distribution measurements were not able to be taken, so instead the ion velocity is estimated by the relation

$$
\frac{1}{2} \mathrm{mv}^{2}=\mathrm{e}\left(\mathrm{V}_{\mathrm{d}}\right)
$$

where $\mathrm{m}$ is the particle mass, $\mathrm{v}$ is the ion velocity, e is the elementary charge, and $\mathrm{Vd}$ is the discharge voltage. By using this equation, the assumption is that the ions are accelerated by the electric field of the anode at an efficiency of $100 \%$. This is likely false, but the assumption was used in the absence of RPA measurements [15].

The actual beam current density as a function of radial distance can then be predicted from the initial collected current with

$$
\mathrm{j}_{\mathrm{b}}(\mathrm{r})=\frac{\mathrm{I}_{\mathrm{c}}}{\mathrm{A}_{\mathrm{F}}\left(\frac{\mathrm{j}}{\mathrm{j}_{0}}\right)_{\mathrm{Ar}^{+}}}
$$

where $A_{F}$ is the collection area of the Faraday probe in $\mathrm{m}^{2}$. Because the RPA was actually used as a Faraday probe, $A_{F}$ was actually the area of the RPA. 


\section{THRUSTER TESTING APPARATUS}

\subsection{VACCUM SYSTEM}

All of the testing for this thesis was performed in the Cal Poly Aerospace Engineering Space Environments Laboratory. The vacuum chamber is a High Vacuum Equipment Corporation (HVEC) 350 liter bell jar chamber, shown in Figure 25. The chamber measures 30 inches in diameter and about 30 inches high. This chamber was used for two reasons: its size and its high-vac capabilities. The large volume of the chamber is desirable so there is sufficient space for the HEMPT's plasma plume to develop without contacting the walls. The high-vac characteristic is necessary to prevent hollow cathode poisoning [17].

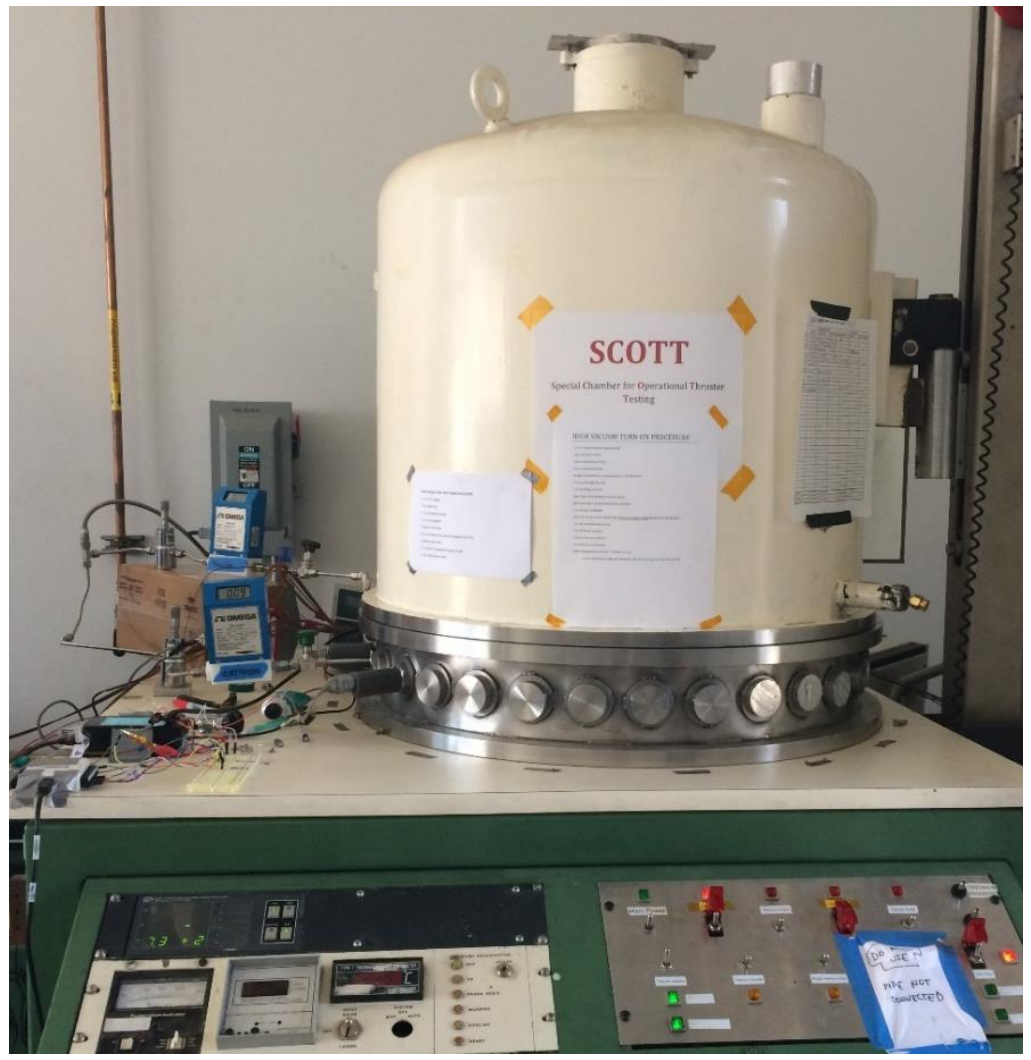

Figure 25. High Vacuum Equipment Corporation (HVEC) 350 Liter Bell Jar Chamber.

Two pumps were used to get this chamber to reach the single-digit $\mu$ Torr pressure necessary to begin testing. First, a Welch 1374 Duo-Seal Vacuum Pump was used to rough the chamber to medium vacuum. This pump is capable of taking the chamber from atmospheric 
pressure down to about 1 mTorr. However, the second pump was generally used to take over in the range of 50-100 mTorr. The second pump is a CTI-Cryogenics Cryo-Torr 8 High Vacuum Pump which is operated with a Model 1020R Helium Compressor. This cryo pump was used to bring the chamber down to $5 \mu$ Torr before initiating Argon flow for thruster and hollow cathode operation. If cathode heating was initiated prior to the low pressure level, it is likely that the cathode insert would be contaminated by impurities in the air. This is known as cathode poisoning, and would make the cathode useless. A schematic of the vacuum system is shown in Figure 26.

Two pressure measurement devices were used for testing. First, from low to medium vacuum, a Granville Phillips 275 convectron gauge was used. When this gauge zeroed out (at $1 \times 10^{-4}$ Torr), the Tubulated Bayard-Alpert 274 ion gauge was switched on. This gauge can measure as low as $1 \times 10^{-9}$ Torr. Both gauges were operated by a Granville Phillips 307 Vacuum Gauge Controller. Because the ion gauge is calibrated for Nitrogen, pressures displayed by the vacuum gauge controller during testing would be inaccurate. To convert these pressures to the equivalent pressure of argon gas, the pressure readings

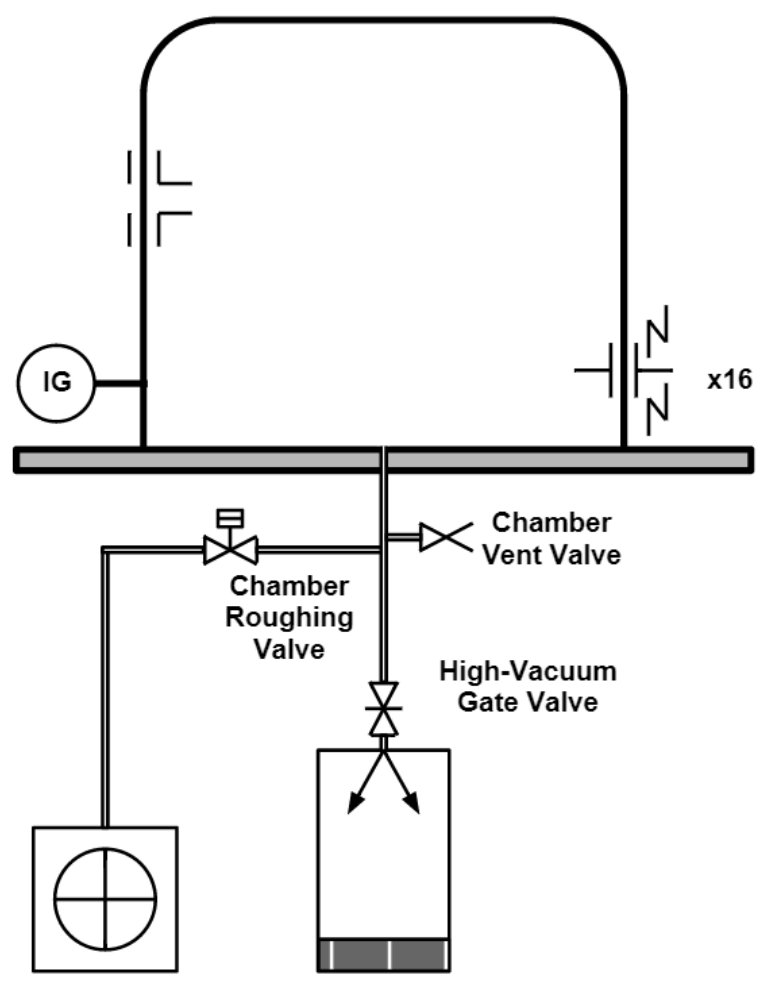

Figure 26. HVEC Vacuum System Schematic. Schematic Courtesy of Scott McGrail [15], reproduced here with permission. were multiplied by 1.29 [18].

The plume of the thruster is composed of highly energetic charged particles. The stainless steel walls of the chamber, if exposed to this plume, could sputter. This would damage the 
chamber and potentially the thruster or cathode if particles from the wall reach them. For this reason, the walls and ceiling of the chamber were covered in $1 / 16$ " thick grafoil sheeting. This material was chosen over carbon felt because it is easier to work with and less porous. Also, carbon felt tends to shed fibers, creating particulate contamination within the chamber.

The vacuum system takes about 30 hours to pump down to $5 \mu$ Torr due to the large volume of the chamber and the relatively small cryo pump. Because of this, it was essential to double check every aspect of the experimental setup before beginning to pump down.

\subsection{LINEAR PROBE DRIVE SYSTEM}

In order to get useful data from the RPA and Faraday probe it was necessary to translate them radially through the thruster plume. This was achieved using a linear probe drive system. The most important component of the system is a stepper motor. This motor is connected to a timing belt, which is connected to the probes' mount. This mount is able to freely slide on 2 stainless steel rails which are aligned perpendicular to the thruster's centerline. A string potentiometer (string pot) is attached to the probes' mount and is used to obtain accurate measurements of the probes' location. An image of the probe drive system is shown in Figure 27. 


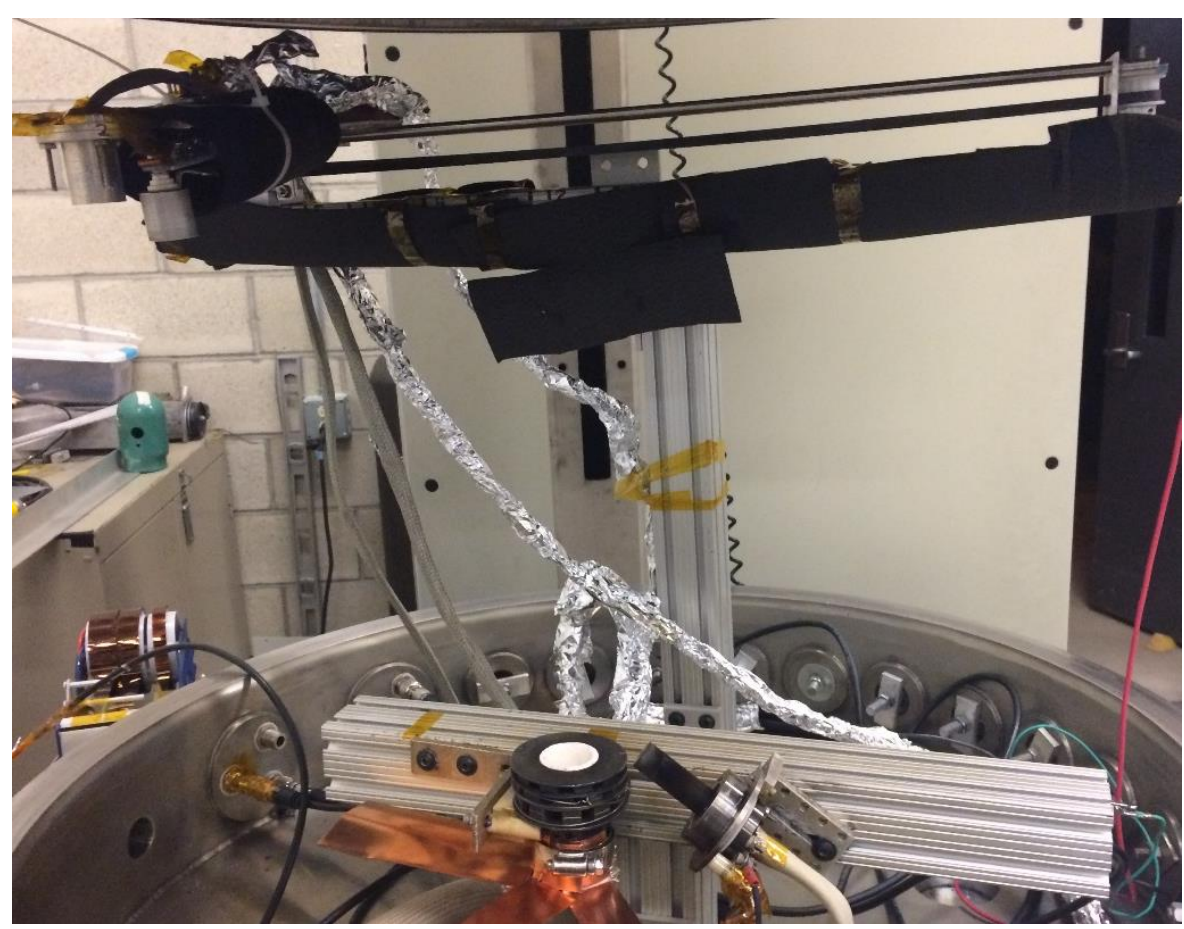

Figure 27. Image of Probe Drive System.

The motor is a Bipolar Mercury Stepper Motor from SparkFun Electronics (SM42BYG011-25) with a step angle of $1.8^{\circ}$ and a holding torque of $2.3 \mathrm{~kg} \cdot \mathrm{cm}$. A Big Easy Driver chip (ROB-12859), also from SparkFun Electronics, was implemented in order to power and control the stepper motor. Additionally, an Arduino Uno microcontroller was used to power and command the motor driver.

The string pot is a Celesco SP-25. It has a measurement range of 0-25 inches. It is a simple measurement device which relies on a set input voltage. For example, if $10 \mathrm{~V}$ is applied to the device then at full extension (25") the string pot's signal terminal will output $10 \mathrm{~V}$. At half extension (12.5"), it will output 5V. This is an essential tool for this experiment because the motor tends to see heavy electromagnetic interference and is often unreliable when commanded to move a certain distance. The string pot gives a very accurate $( \pm 1 \%)$ location measurement of the probes. Previous experiments prior to the use of a string pot relied on visual inspection of the RPA's location via the chamber's small window, resulting in significant uncertainty. This was on top of the fact that the probe drive had a tendency to "jitter" due to EM noise in previous 
experiments. This jitter was eliminated in this series of experiments. The RPA and Faraday probe will be swept perpendicularly to the thruster's centerline in a continuous motion.

Due to the previously mentioned EM interference experienced by the motor, the entirety of the motor leads were shielded by twisting the wires around each other and covering them in a metallic overbraid to provide EMI shielding.

\subsection{DATA COLLECTION SYSTEM}

A Student Edition of LabVIEW 2012 and a NI USB-6009 DAQ were used for data collection. The 6009 DAQ is a 14-bit system with 8 analog inputs, each limited to $10 \mathrm{~V}$ or less. It was used to acquire data, including RPA collector current, Faraday probe current, string pot position, RPA discriminator grid voltage, cathode flow rate, thruster flow rate, ion gauge pressure, convectron gauge pressure, keeper voltage, and keeper current. The DAQ is used for reading voltages, but useable data from both the RPA and Faraday probe are expressed as a current. Because of this, two sense resistors were added: one between the RPA collector and ground and one between the Faraday probe collector and ground. The DAQ measures voltage

drops across these resistors, which can be easily converted to currents. In order to reduce noise on the probe lines induced by the plasma and high voltage supplies, a capacitor was added to the RPA collector circuit. A schematic of this setup is shown in Figure 28. 

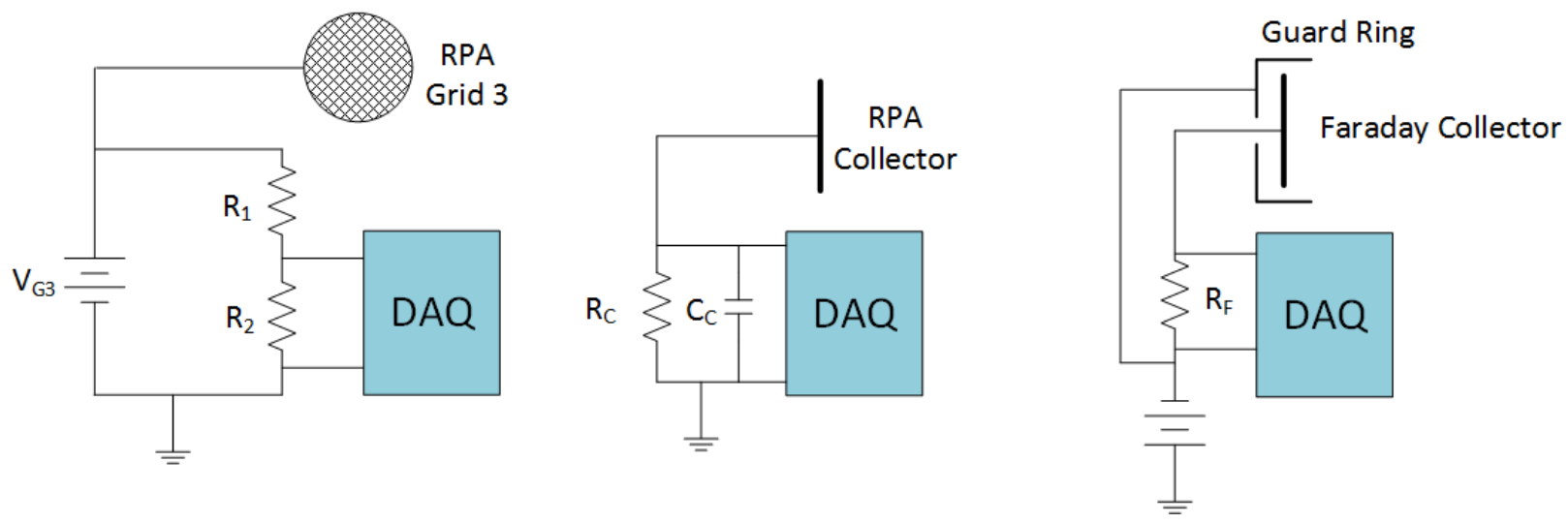

Figure 28. Electrical Diagram of RPA Collector and Faraday Probe.

The values for the resistors and capacitors in Figure 28 were not arbitrarily chosen. The fact that the DAQ is 14-bit means that it can read voltage data in $2^{14}$ steps, or 16,384 steps. Because the DAQ's max input is $10 \mathrm{~V}$, this means that each step represents $\frac{10 \mathrm{~V}}{16384}$, or about $0.6 \mathrm{mV} / \mathrm{step}$. Therefore in order for the DAQ to register a signal it has to be above $0.6 \mathrm{mV}$. This could be potentially problematic for the RPA and Faraday probes, which see in the neighborhood of $1-10 \mu \mathrm{A}$ and 1-2mA, respectively [7]. To remedy this, resistor values were chosen so that the DAQ would see acceptable voltages. It is generally good practice not to approach the minimum step, as resolution decreases with measurements close to it. For example, changes to a $6 \mathrm{mV}$ signal can only be in sizes of $0.6 \mathrm{mV}$, or $10 \%$. Therefore, a $10 \mathrm{x}$ margin was included above the minimum step size. To account for plasma discharge noise, another margin of 10x the minimum step was included. This means that the minimum acceptable voltage signal seen by the DAQ was $60 \mathrm{mV}$. For the RPA's discriminator grid, the issue was the opposite. The grid is swept from 0$500 \mathrm{~V}$, well above the DAQ's upper limit. This was fixed using a voltage divider circuit. It was also important that the resistance for this voltage divider was high enough that it did not draw significant current because the discriminator grid's supply could only support $125 \mathrm{~mA}$.

The limiting factor for the size of the capacitors was the RC time constant, $\tau$, which represents the minimum acceptable time between measurements. If $\tau$ was to large, it would take 
too long to take measurements across the plume. The chosen components to meet or exceed all of these requirements are summarized in Table 7.

Table 7. Resistance and Capacitance Values for Data Collection.

\begin{tabular}{cl}
\hline Component & Value \\
\hline $\mathrm{R}_{1}$ & $22000 \Omega$ \\
$\mathrm{R}_{2}$ & $100 \Omega$ \\
$\mathrm{R}_{\mathrm{F}}$ & $220 \Omega$ \\
$\mathrm{R}_{\mathrm{C}}$ & $146 \mathrm{k} \Omega^{*}$ \\
$\mathrm{C}_{\mathrm{C}}$ & $0.68 \mu \mathrm{F}$
\end{tabular}

*For the RPA Collector, the built-in impedance of the DAQ $(146 \mathrm{k} \Omega)$ was sufficient for reading current from $1-50 \mu \mathrm{A}$, so no additional resistance was added ( $\mathrm{R}_{\mathrm{C}}$ is technically 0$)$

A LabVIEW VI was created to handle the data collection from the 6009 DAQ. The front panel of this VI is shown in Figure 29. When the Record State button is pressed, all data displayed on the front panel is written to a log file. This data is all that is necessary to characterize the thruster's performance, with the exception of discharge voltage and discharge current. These two were not included because the DAQ only had 8 analog input channels. It was possible to collect 2 more channels worth of data (for a total of 10), however, because the keeper's voltage and current were the results of direct queries to the supply using an Ethernet crossover cable and SCPI commands. This could have been done for the discharge supply as well, but the laptop used for testing had only one network port. A connection via USB is also a possibility, but the supplies were located about 7 feet from the data collection point and no USB cable this long was available at the time. Also, both of the computer's USB ports were in use (Arduino Board and DAQ). Because of this, data from the discharge supply was collected using still images and then later transcribed after data was collected. A more detailed look at the LabVIEW code behind the front panel is presented in Appendix 0. 


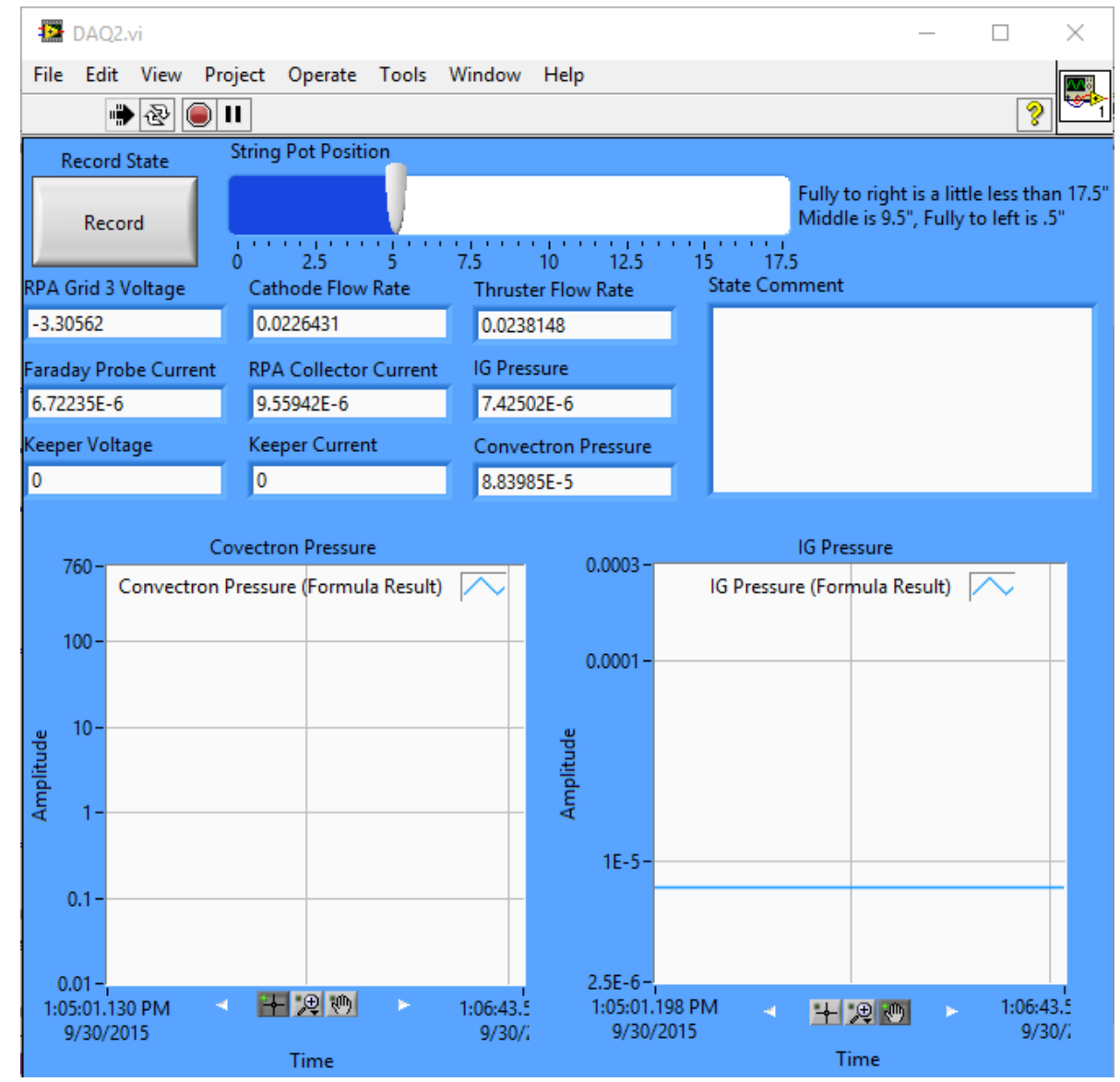

Figure 29. LabVIEW VI Front Panel.

Video and still images of the thruster were collected during testing via a GoPro Hero 4 camera mounted on the window at the back of the chamber. The live video feed was sent via WiFi to an iPad which was placed near the power supplies. This enabled a very clear understanding of what was happening inside of the chamber, which was particularly useful while attempting to light the cathode and start the thruster.

\subsection{ELECTRICAL SYSTEM}

The electrical system for this experiment was fairly complex. It involved the use of 8 separate power supplies which are detailed in Table 8. 
Table 8. List of Power Supplies Used for HEMPT Testing.

\begin{tabular}{lll}
\hline Application & Power Supply & Output \\
\hline Discharge & Sorenson XG 300-5.6 & $0-300 \mathrm{~V}, 0-5.6 \mathrm{~A}$ \\
Faraday Probe & Extech Inst. 382202 & $0-18 \mathrm{~V}, 0-3 \mathrm{~A}$ \\
Heater & HP 6038A & $0-60 \mathrm{~V}, 0-10 \mathrm{~A}$ \\
Igniter/ RPA Grid 3 & Glassman FC1P120 & $0-1000 \mathrm{~V}, 0-125 \mathrm{~mA}$ \\
Keeper & Agilent N5771A & $0-300 \mathrm{~V}, 0-5 \mathrm{~A}$ \\
Motor Controller & Extech Inst. 382202 & $0-18 \mathrm{~V}, 0-3 \mathrm{~A}$ \\
RPA Grid 2 & HP 6263B & $0-24 \mathrm{~V}, 0-12 \mathrm{~A}$ \\
String Potentiometer & Instek GPS 2303 & $0-30 \mathrm{~V}, 0-3 \mathrm{~A}$ \\
\hline
\end{tabular}

The Glassman FC1P120 was used for two purposes. First, it was used to help light the cathode. The hollow cathode often required upwards of $400 \mathrm{~V}$ to initiate plasma generation, which is much higher than the Keeper's $300 \mathrm{~V}$ maximum. Therefore, the positive terminals of the supplies were connected to each other with $1000 \mathrm{~V}, 6 \mathrm{~A}$ diodes between each supply and the connection point. This ensured that both supplies were protected from each other by allowing current to only flow out of the supplies. After hollow cathode ignition, the Glassman power supply was then used to bias RPA grid 3, the discriminator grid. This was done using a switch.

The vacuum chamber system has 8 dedicated coaxial electrical feedthroughs. This includes 3 TNC and $5 \mathrm{~N}$-type feedthroughs. This experiment, however, required 14 isolated feedthroughs. This was achieved using thermocouple wires to carry low-current signals. The chamber is equipped with a single feedthrough of 25 thermocouples which was more than sufficient for this experiment. The feedthrough connections are detailed in Table 9. 
Table 9. Electrical Feedthrough Connections.

\begin{tabular}{lcll}
\hline Device & \# of Feedthroughs & Specific Connections & Type of Connection \\
\hline HEMPT & 1 & Discharge & Coaxial \\
RPA & 3 & Grid 2, Grid 3, Collector & Coaxial \\
Faraday Probe & 2 & Collector, Guard Ring & Coaxial \\
Hollow Cathode & 2 & Heater, Keeper/Igniter & Coaxial \\
Stepper Motor & 4 & Power and Signals (Bipolar Motor) & Thermocouple \\
String Potentiometer & 2 & Power and Signal & Thermocouple \\
\hline
\end{tabular}

The schematic of the entire electrical system for the HEMPT, hollow cathode, and all accessory devices needed for testing is shown in Figure 30.

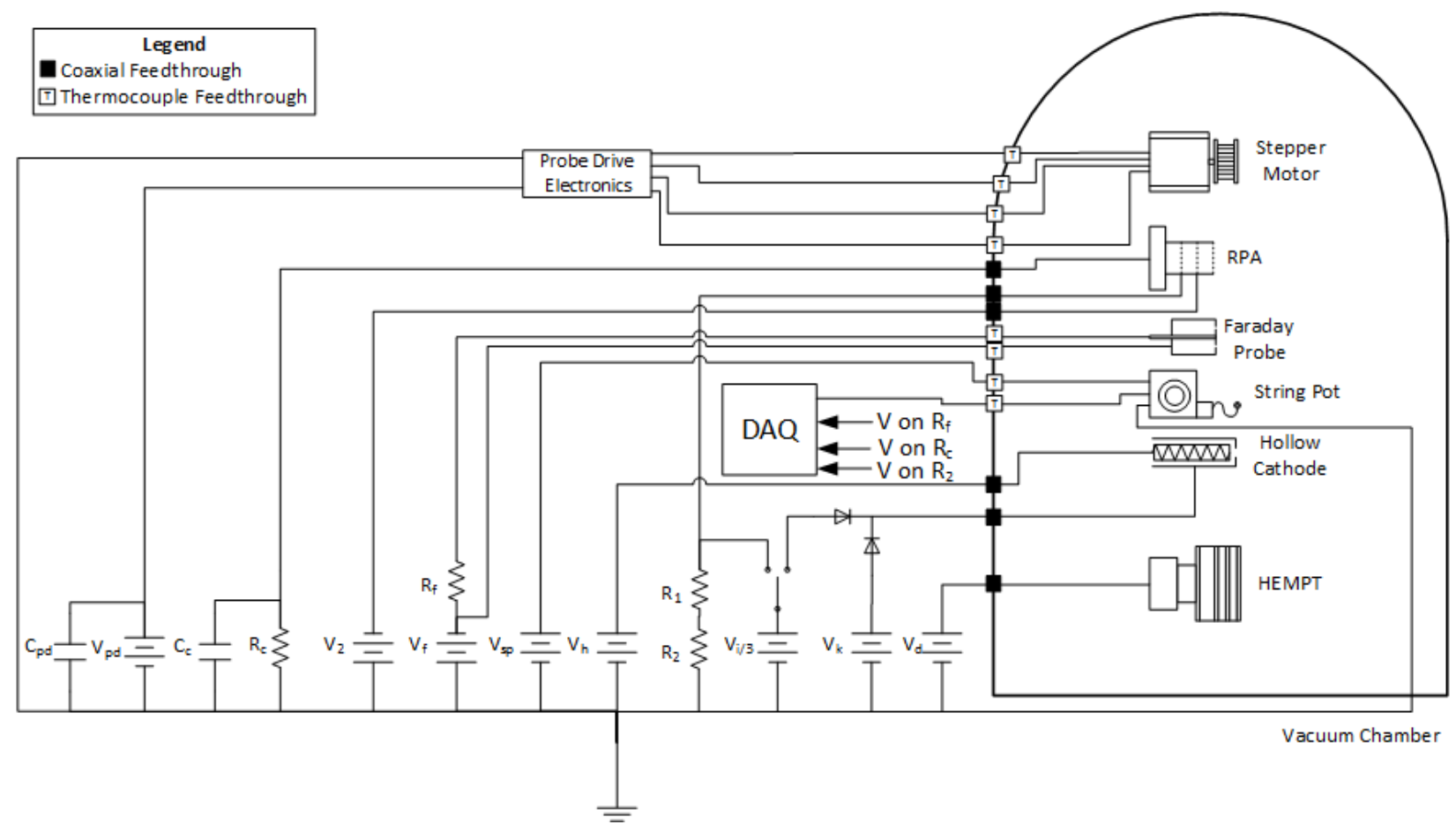

Figure 30. Electrical Schematic for HEMPT Testing.

Because of the complexity of the electrical system and the high voltage needed for many of the devices, special attention was paid to the electrical isolation and EMI shielding of all components. All signal-carrying wires within the chamber were wrapped in aluminum foil and aluminized tape to protect them from EMI. The exception to this was the coaxial cables from the 8 chamber feedthroughs. The inherent geometry of coaxial cables provides sufficient protection 
from EMI. The discharge line (which connects the back of the HEMPT to the propellant electrical isolator) was wrapped in insulative ceramic mesh tubing. This was done to protect the discharge line (which is at high positive potential of $200+\mathrm{V}$ ) from attracting the plasma, which would result in performance decreases as well as thermal issues. Previous testing used Kapton sheets and Kapton tape instead of ceramic mesh, but at least one of these tests caused the Kapton to melt, which was detrimental to thruster performance. Likewise, the hollow cathode's propellant line between the cathode and the isolator was also covered in ceramic mesh tubing.

\subsection{PROPELLANT FEED SYSTEM}

Thrusters (at least current models) need to eject propellant to produce thrust. The HEMPT needs an inert gas which can be ionized and electrostatically accelerated. Xenon is a good choice for this due to its relatively high mass and low ionization energy. However, it is also more expensive than some alternatives. For this reason, argon gas is used for testing here. Argon is less massive than xenon and takes more energy to ionize, but is less expensive to obtain for use in testing [19]. Ultra high purity argon was necessary for use in this thesis. This means the argon is $99.999 \%$ pure, a purity level necessary to prevent the hollow cathode insert from poisoning [17].

The propellant feed system for this thesis consists of a tank of UHP argon, a two-stage pressure regulator, two needle valves, two propellant flow meters, two manual shut-off valves, two chamber gas feedthroughs, and two propellant electrical isolators. All of these components are connected using $1 / 4$ " stainless steel tubing with Swagelok connectors.

Both needle valves were VACOA MV-25 Precision Micrometer Gas Metering Valves which were used to precisely control the flow rate of argon through the thruster and cathode. The flow meters were OMEGA FMA-A2300's with digital displays and output in units of standard cubic centimeters per minute of $\mathrm{N}_{2}\left(\mathrm{SCCM}\left[\mathrm{N}_{2}\right]\right)$. These flow meters, like the ion gauge used to measure chamber pressure, are calibrated for nitrogen gas. Therefore, a conversion factor of 1.45 
was used to get flow rate readings for argon gas with an accuracy of $\pm 4 \%$ [20]. After the flow meter on each propellant line there are Swagelok SS-4H bellows-sealed manual shut-off valves. These valves are used to separate the chamber from the gas lines whenever the system is not in use. Finally, a propellant electrical isolator is placed on each line, just before the thruster and cathode. This ensures that the high voltages sent to these two devices are isolated from the chamber, which is grounded. A schematic of the propellant flow system is shown in Figure 31.

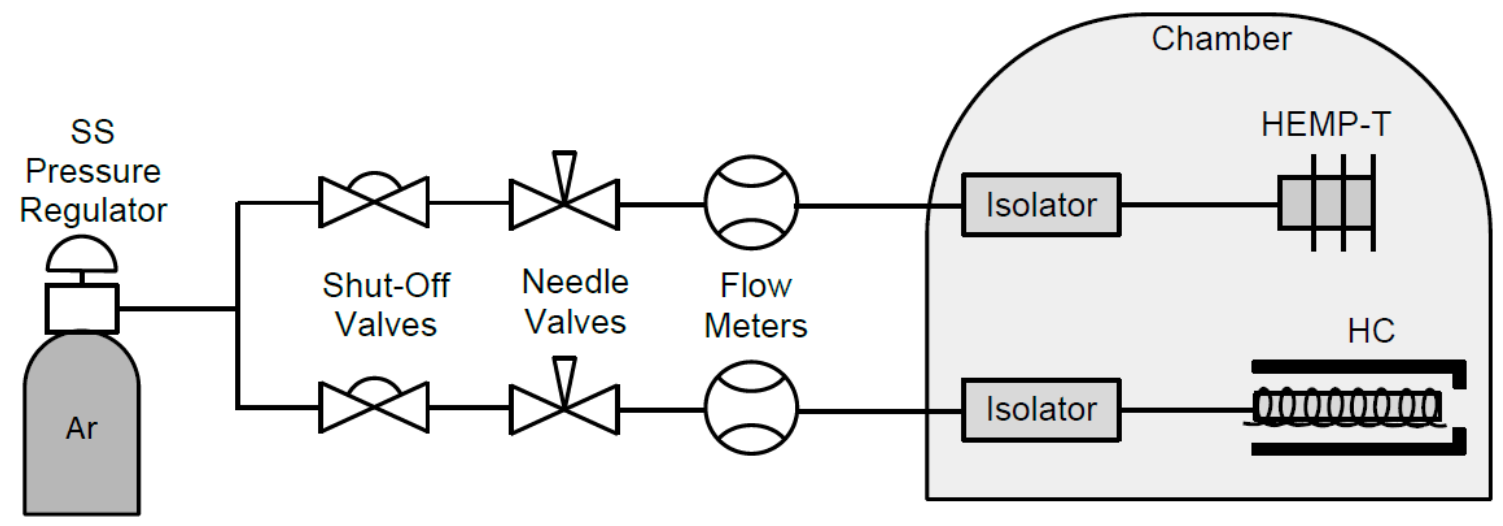

Figure 31. Propellant Feed System Schematic for HEMPT and Hollow Cathode. Image courtesy of Scott McGrail, reproduced here with permission [15]. 


\section{CALCULATING THRUSTER PERFORMANCE}

There are many metrics for quantifying and comparing EP thruster performance, but perhaps the three most important and widely used are thrust produced, specific impulse, and total efficiency. This section outlines the steps taken to calculate these quantities based on measured data. Total efficiency is calculated first, followed by thrust and specific impulse.

Prior to the calculation of these metrics, an important intermediate parameter, particularly for this thesis, is the plume's half-angle divergence. The half-angle divergence can be defined in several ways. However, in order to maintain consistency with Scott McGrail's thesis, the same definition he used will be repeated here. The divergence half-angle, $\theta$, is defined as the angle which encloses $95 \%$ of the calculated beam current, per Hofer and other experts in various Hall thruster experiments [21].

\subsection{THRUST AND TOTAL THRUSTER EFFICIENCY}

In order to calculate total thruster efficiency, many intermediate values must first be obtained. The first of these is the beam current fraction of discharge, $\eta_{b}$, which is given by,

$$
\eta_{b}=\frac{I_{b}}{I_{d}}
$$

where $I_{b}$ is the beam current, obtained from Eqn. 12, and $I_{d}$ is the discharge current of the thruster [1]. The next efficiency ratio is the beam voltage fraction of discharge, $\eta_{v}$, which is calculated very similarly,

$$
\eta_{v}=\frac{V_{b}}{V_{d}}
$$

where $V_{b}$ is the beam voltage given by Eqn. 11 and RPA data, and $V_{d}$ is the discharge voltage of the thruster [1].

Because not all of the propellant that is pumped into the thruster's channel is ionized, an efficiency term must be introduced to account for this. This is the thruster mass utilization efficiency, $\eta_{m}$, and is given by 


$$
\eta_{m}=\alpha_{m}\left(\frac{I_{b} M_{A r}}{m_{p} e}\right)
$$

where $\alpha_{m}$ is a term added to account for the doubly charged ions [1]. For this thesis, a 5\% fraction of doubly charged ions is assumed to exist in the plume based on initial test data from Thales Electron Devices [6]. $\alpha_{m}$ is given by,

$$
\alpha_{m}=\frac{1+\frac{1 I^{++}}{2 I^{+}}}{1+\frac{I^{++}}{I^{+}}}
$$

where $\frac{I^{++}}{I^{+}}$is the fraction of doubly charged ions in the thruster's beam [1].

The thruster is not completely electrically efficient. Not every watt of power that is put into the thruster translates directly into the beam because much of the energy is lost to heat or to plasma generation via the cathode keeper. This inefficiency is accounted for with $\eta_{0}$,

$$
\eta_{0}=\frac{I_{b} V_{b}}{I_{b} V_{b}+P_{k}}
$$

where $P_{k}$ is the keeper power, expressed in Watts [1].

The thrust correction factor is then used to correct for the additional thrust force which doubly charged ions produce when accelerated by a voltage, and is given by,

$$
\alpha=\frac{1+0.707 \frac{I^{++}}{I^{+}}}{1+\frac{I^{++}}{I^{+}}}
$$

Based on $\alpha$ and the previously discussed plume divergence half angle, $\theta$, the total thrust correction factor can be calculated, and is given by [1] ,

$$
\gamma=\alpha \cos (\theta)
$$

The total thruster efficiency is a product of the previously discussed efficiency, and is given by [1],

$$
\eta_{T}=\gamma^{2} \eta_{b} \eta_{v} \eta_{m} \eta_{0}
$$

Note that $\eta_{T}$ is proportional to $\gamma^{2}$, whereas the other efficiency terms come into play only once. This indicates that the divergence half angle plays a very important role in determining thruster performance. Finally, the thrust produced by the HEMPT is then given by, 


$$
T=\gamma \sqrt{\frac{2 M}{e}} I_{b} \sqrt{V_{b}}
$$

\subsection{SPECIFIC IMPULSE}

Before calculating $I_{s p}$, it is necessary to account for the fact that this thruster is being operated in a laboratory environment. Specifically, the HEMPT is operating in a confined volume. A major side effect of this is that the environment immediately surrounding the thruster will be under some level of pressure due to the excess argon in the chamber. The thruster can then pull in this excess Argon and use it to produce ions. In the relatively small chamber used for testing in this thesis, this effect, known as ingestion, can be fairly significant. To account for this, the mass flow rate of propellant must be adjusted. To do this, first the flow rate balance equation is considered,

$$
Q_{\text {total }}=Q_{\text {injected }}+Q_{\text {ingested }}
$$

where $Q$ is the volumetric flow rate of argon, expressed in standard cubic centimeters per minute $(\mathrm{sccm})$ [1]. Note that the injected flow rate is the combined amount of argon fed to both the cathode and thruster. This value is the sum of the values read off of the flow meter for each device (with an argon correction factor applied). The ingested flow is slightly more complicated, and is given by,

$$
Q_{\text {ingested }}=7.82 \times 10^{8} \frac{P A \eta_{c}}{\sqrt{T M_{a}}}
$$

where $P$ is the vacuum chamber pressure in Torr, $T$ is the temperature of the backflowing neutral gas in $K, M_{a}$ is the gas atomic mass in AMU, and $A$ is the open area of the thruster in $m^{2} . \eta_{c}$ is a correction factor to account for the conductance into the thruster, but this is often assumed to be 1 and will be for this thesis [15]. The open area, $A$, is generally equal to the exit area of the thruster, but experiments have shown that for the HEMPT, because much of the acceleration occurs outside of the thruster, the open area is effectively larger. Because of this, the value of $A$ will be 
approximated by a hemisphere with the same diameter as the ionization channel. The corrected mass flow rate, incorporating the conversion from sccm to $\mathrm{kg} / \mathrm{s}$ of argon, is then given by,

$$
m_{p}=2.725 \times 10^{-8} \cdot Q_{\text {total }}
$$

With this final parameter, the $I_{s p}$ of the thruster can be calculated by,

$$
I_{s p}=\frac{T}{m_{p} g_{0}}
$$

Equations 27, 24, and 23 will provide the three main metrics which will be used to describe the HEMPT's performance before and after the addition of a magnetic shield. 


\section{TESTING CONDITIONS}

Three tests in which useable data were collected were performed. Test 1 was the baseline configuration, unshielded. Test 2 was with the shield. Test 3 was once again unshielded, this time with the RPA used as a Faraday probe to collect the ion current profile.

Ideally a setpoint of HEMPT operation that could be visited repeatedly and reliably in both the shielded and unshielded configuration would be found. This stable operating point is defined by a combination of argon flow rates to the HEMPT and cathode, discharge voltage and current, and keeper voltage and current. The vacuum chamber back pressure is also an important parameter that affects performance, but it is taken into account via the adjustment applied to the mass flow rate, $\dot{m}_{p}$, because backpressure isn't as easily controllable as the other parameters. However, it proved difficult to find a single setpoint that could be easily revisited. Instead, the parameters for each test are summarized in the tables that follow. The setpoint for the first test, the unshielded HEMPT with the Faraday probe, is outlined in Table 10.

\begin{tabular}{ll} 
Table 10. Test 1: Unshielded HEMPT Operation Parameters. \\
\hline Setpoint Parameter & Value \\
\hline HEMPT Flow Rate & $9.21 \pm 0.09$ SCCM Argon \\
Cathode Flow Rate & $22.48 \pm 0.22$ SCCM Argon \\
Discharge Power & $235.2 \pm 0.3 \mathrm{~V}, 2.22 \pm 0.01 \mathrm{~A}$ \\
Keeper Power & $42.7 \pm 0.8 \mathrm{~V}, 2.508 \pm 0.001 \mathrm{~A}$ \\
\hline
\end{tabular}

However, for the shielded test run, the HEMPT was not able to reach this same setpoint. Instead, the operation parameters for the shielded test are summarized in Table 11. The most important difference is the discharge power, which wasn't able to climb to the same voltage as its unshielded counterpart. 
Table 11. Test 2: Shielded HEMPT Operation Parameters.

\begin{tabular}{ll}
\hline Setpoint Parameter & Value \\
\hline HEMPT Flow Rate & $7.64 \pm 0.08$ SCCM Argon \\
Cathode Flow Rate & $23.44 \pm 0.23$ SCCM Argon \\
Discharge Power & $192.4 \pm 0.3 \mathrm{~V}, 2.53 \pm 0.01 \mathrm{~A}$ \\
Keeper Power & $39.21 \pm 0.8 \mathrm{~V}, 2.508 \pm 0.001 \mathrm{~A}$ \\
\hline
\end{tabular}

It is clear that the difference in setpoint values between the first two tests was due to the addition of the shield because when the shield was removed for test 3 the values were much more similar to those of test 1 . These values are summarized in Table 12.

Table 12. Test 3: Unshielded HEMPT Operation Parameters.

\begin{tabular}{ll}
\hline Setpoint Parameter & Value \\
\hline HEMPT Flow Rate & $8.97 \pm 0.09$ SCCM Argon \\
Cathode Flow Rate & $23.06 \pm 0.23$ SCCM Argon \\
Discharge Power & $236.6 \pm 0.3 \mathrm{~V}, 2.21 \pm 0.01 \mathrm{~A}$ \\
Keeper Power & $40.1 \pm 0.8 \mathrm{~V}, 2.508 \pm 0.001 \mathrm{~A}$ \\
\hline
\end{tabular}

Also, in all cases the keeper power was higher than would be expected. Normally, this hollow cathode runs below $30 \mathrm{~V}$ at 1-3A. It is possible that the position of the cathode relative to the thruster caused increased electron impedance, resulting in higher voltage. However, due to time constraints, the reason for the higher voltage was not fully explored. This increased power draw negatively impacted thruster performance, but this thesis was more interested in the delta in performance due to the addition of a shield rather than the absolute performance. 


\section{RESULTS}

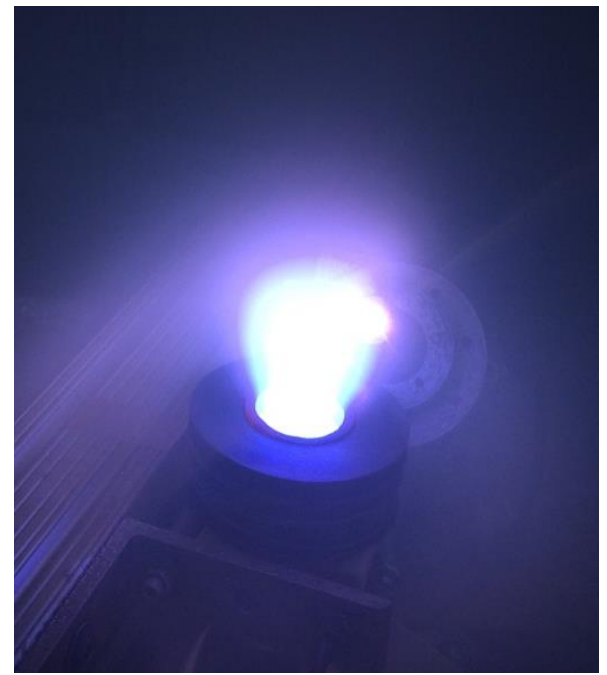

Figure 32. Unshielded HEMPT in Operation.
The results of HEMPT testing in the shielded and unshielded configurations are presented here.

Unfortunately, the combination of a number of issues resulted in data which is less useful than was originally planned. First, the chamber background pressure was fairly high due to the use of Argon in place of Xenon. The cathode and thruster required significantly higher flow rates of gas than in previous tests with Xenon.

The second problem was that the nude Faraday

probe, which was intended to collect ion current and generate a plume profile, proved to be almost completely overwhelmed by slow-moving ions produced from charge exchange interactions in background neutrals. This was due to the relatively high chamber background pressure, and is a known limitation for nude Faraday probes [22]. The net result was exaggerated collected ion current at the edges of the plume, which meant a false and nonsensical calculated total beam current. Because of this, the Faraday probe measurements were mostly unusable.

The third problem arose when the RPA was first used to measure the beam voltage at the beam's centerline. The RPA wasn't behaving properly, and after further investigation it was determined a short was present between grids. When the RPA was disassembled there was a visible hole in grid 3 and a matching impression in grid 2. It appears that when the voltage of grid 3, the discriminator, was ramped up to around $400 \mathrm{~V}$ a discharge occurred, damaging both grids. The edges of the hole on grid 3 were bent outward such that when the RPA was assembled grids 2 and 3 made electrical contact. The improvised, short term solution to this issue was to place a ring of kapton sheeting between the grids so there would be just enough spacing to prevent current from flowing. This inevitably resulted in an increased grid transparency which would affect any measurements taken by the RPA. This increased transparency was not calculated as the 
diameter of the hole was not measured. Also, the RPA still didn't work properly and a beam voltage measurement was never obtained.

Because of the aforementioned issues with both the Faraday probe and RPA, neither was used for their intended purposes. The RPA was instead used as a Faraday probe by biasing grid 2 below the plasma potential and applying no voltage to the other grids. Though this is not its intended use, it works quite well because the RPA structure is similar to that of a collimated Faraday probe, the preferred form of ion current measurements when background pressure is high. It was with this improvised collimated Faraday probe that an actual plume profile was collected. For future experiments, a collimated Faraday probe should be built or obtained. The use of xenon in place of argon may also prevent the pressure from rising high enough to blind the Faraday probe.

\section{UNSHIELDED HEMPT RESULTS}

The unshielded HEMPT was the first configuration studied in order to obtain baseline measurements and characterize the HEMPT before making changes to its magnetic field. First, the nude Faraday probe was used in an attempt to create an ion current profile of the plume. This was done by biasing the probe to $-20 \mathrm{~V}$ and sweeping it slowly across the plume using the linear probe drive. The DAQ and LabVIEW were used to measure the current from the probe in real time. Four sweeps were performed during the same run, and the data is plotted in Figure 33. Each color represents one plume sweep, and in each case a significant dip in current is detected at the thruster centerline. As previously mentioned, charge exchange interactions resulted in exaggerated current away from the centerline, so this data is not sufficient to calculate total beam current. However, it is useful for the fact that it shows behavior consistent with a hollow plume. 


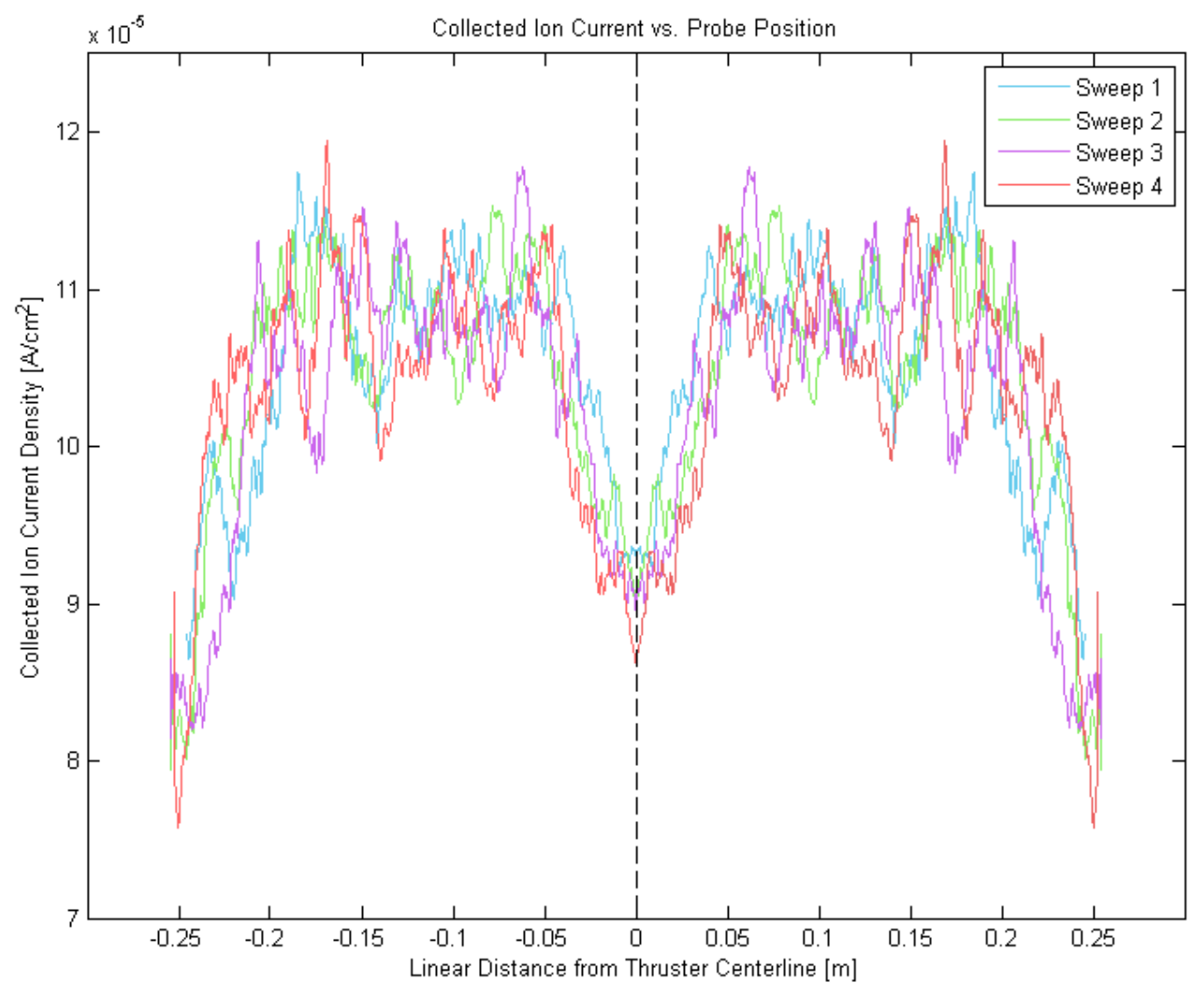

Figure 33. Unshielded HEMPT Ion Current Plume Profile with Faraday Probe.

The next step was to rerun the thruster and use the RPA in its Faraday probe configuration. This resulted in much cleaner data which showed both a dip in ion current at the thruster's centerline and very low ion current far from the centerline. The data for three sweeps is shown in Figure 34. The sweeps are labelled chronologically, with Sweep 1 first. Notice the collected current increases with each sweep. This is attributable to increasing chamber background pressure. Sweep 1 was started at a background pressure of $2.82 \times 10^{-4}$ Torr. By the time Sweep 3 was completed, the chamber pressure had risen to $3.73 \times 10^{-4}$ Torr. Each sweep took about 1 minute, so the rate of pressure increase was not negligible. While the RPA is good at rejecting CEX ions from being measured, it is not perfect. Background pressure increases do have an effect on the measured current. 
It is also worth noting that the HEMPT began to show signs of instability during Sweep 2, and was flickering in and out of operation frequently during Sweep 3. This can be seen in Figure 34 by the many spikes and dips in current during Sweep 3. It's possible that this behavior is attributable to the HEMPT overheating. This could be a sign of the permanent magnets becoming degaussed, reducing their ability to contain the plasma. This would allow electrons to stream towards the anode unimpeded, possibly causing this unsteady operation. After this test, the thruster was examined and it was determined that it had indeed suffered significant heat damage to the magnets, and the anode showed signs of significant sputtering. This was the last test during which the HEMPT was able to operate properly.

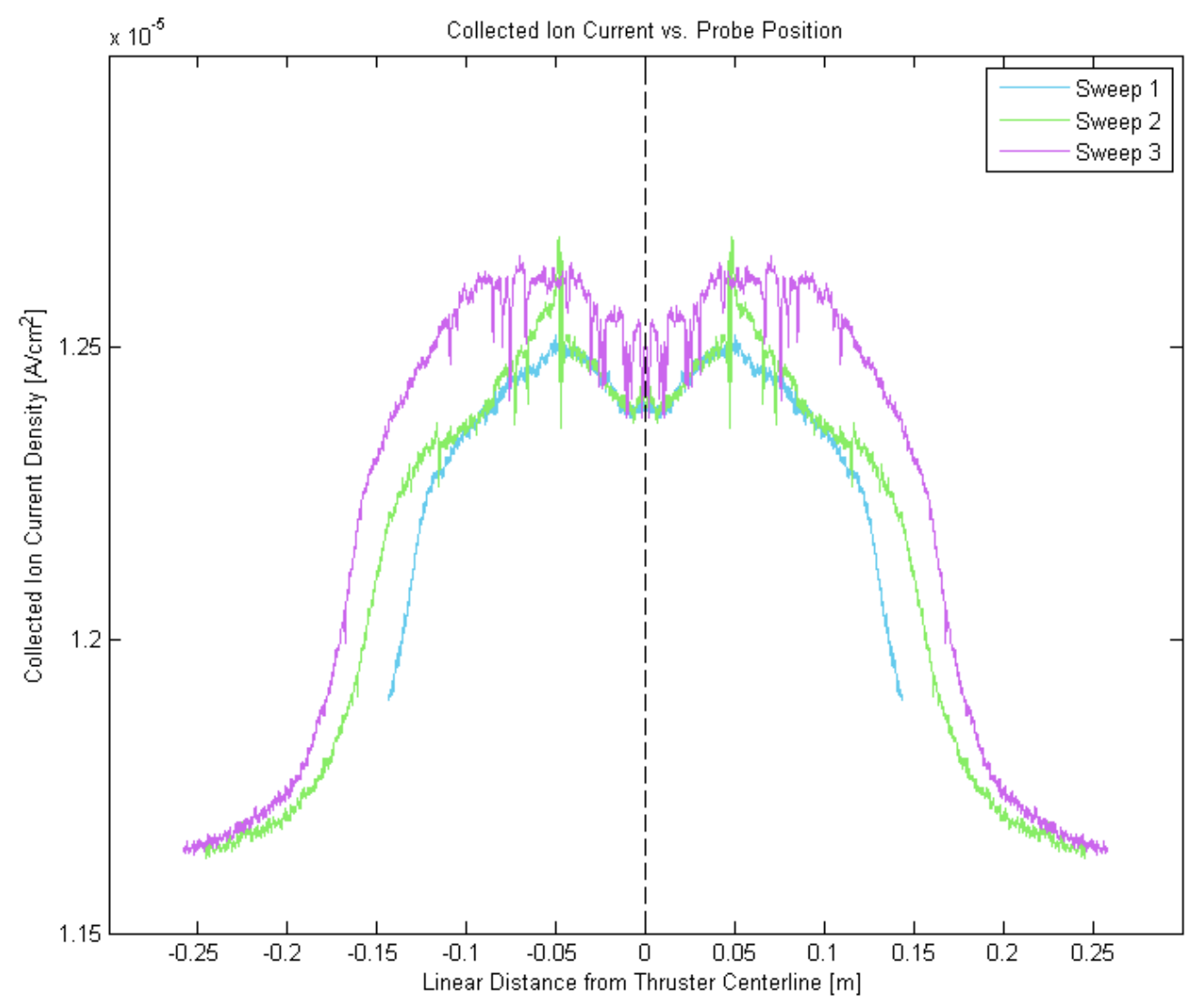

Figure 34. Unshielded HEMPT Ion Current Plume Profile with RPA.

If $11.6 \mu \mathrm{A}$ (the measurement at the edge of the plume) is taken as the zero point, the data show a peak to trough decrease in collected current of 16\%, 21\%, and 27\% for Sweeps 1, 2, and 3, respectively. Using $11.6 \mu \mathrm{A}$ as the zero point is reasonable because the current tapers off and 
asymptotically approaches a value near $11.6 \mu \mathrm{A}$. These percent decreases are very significant. If they could be remedied the thruster's performance should increase substantially.

The peak collected current occurs at a $10.2^{\circ}$ offset. This is less than the $20^{\circ}$ initially predicted by Scott McGrail, but higher than the $4.5^{\circ}$ offset he measured [15]. One explanation for this may be the fact that he measured a small set number of evenly spaced discrete points within the plume. Additionally, his set up resulted in the probe drive physically moving the probes back and forth due to noise on the lines. In contrast, this data was collected using smooth, continuous sweeps with 1000s of points and the signal lines were EMI shielded via twisted pairs and a metallic over braid. This resulted in cleaner data, and much more of it, meaning the maximum and minimum currents could be more accurately identified.

The next step was to obtain the beam current. This was done using Equations 12-15. The CEX correction factor was calculated to decrease from 0.578 to 0.2579 as pressure rose throughout the length of the test. The grid transparency was calculated at 0.0366 [15]. As previously mentioned, the grid transparency was not adjusted to account for the hole in grid 3. The value for the open area of the RPA was $7.56 \times 10^{-5} \mathrm{~m}^{2}[15]$. Based off of these values and a $10 \%$ neutral depletion in the beam due to ionization, the beam current, $\mathrm{I}_{\mathrm{b}}$, was found to be $1.36 \mathrm{~A}$.

Plume divergence half angle is defined by the angle below which $95 \%$ of the calculated ion beam current is collected. The divergence half angle for this HEMPT was $41.3^{\circ}$. This is higher than the $34.3^{\circ}$ divergence calculated by Scott McGrail [15], which again may be attributable to his discrete data collection method. The calculated values are summarized in Table 11. 
Table 13. Unshielded HEMPT Test Results.

\begin{tabular}{lll}
\hline Performance Parameter & Symbol & Value \\
\hline Beam Voltage & $V_{b}$ & $177.5 \mathrm{~V} \pm 23.7 \mathrm{~V}$ [Assumed] \\
Beam Current & $I_{b}$ & $1.36 \mathrm{~A} \pm 0.08 \mathrm{~A}$ \\
Beam Divergence Half Angle & $\theta$ & $41.3^{\circ} \pm 2.7^{\circ}$ \\
Beam Current Fraction of Discharge & $\eta_{b}$ & $61.54 \% \pm 3.62 \%$ \\
Beam Voltage Fraction of Discharge & $\eta_{v}$ & $75 \% \pm 10 \%$ [Assumed] \\
Mass Utilization Efficiency & $\eta_{m}$ & $60.70 \% \pm 3.75 \%$ \\
Electrical Efficiency & $\eta_{0}$ & $70.64 \% \pm 20.63 \%$ \\
Total Thrust Correction Factor & $\gamma$ & $73.28 \% \pm 4.71 \%$ \\
Corrected Mass Flow Rate & $m_{p}$ & $9.06 \times 10^{-7} \pm 0.12 \times 10^{-7} \mathrm{~kg} / \mathrm{s}$ \\
Total Thruster Efficiency & $\eta_{T}$ & $10.63 \% \pm 3.66 \%$ \\
Thrust & $T$ & $12.1 \mathrm{mN} \pm 1.3 \mathrm{mN}$ \\
Specific Impulse & $I_{s p}$ & $1361 \mathrm{~s} \pm 147 \mathrm{~s}$ \\
\hline
\end{tabular}

In comparison to Scott's results for the same thruster a number of differences exist.

Firstly, beam current here is higher than his $1.25 \mathrm{~A}$ because the thruster was run at a higher power set point. What's more important is that the beam current fraction of discharge is much lower than his calculation of $78 \% \pm 9 \%$. This suggests that the thruster may be less efficient when the discharge power is increased. However, this result is not consistent with the behavior Thales Electron Devices observed. It could be, instead, that at higher discharge voltages more power is converted to heat due to the design of this particular HEMPT. More sophisticated HEMPT tests use liquid cooling to allow for much higher discharge powers.

Of course, beam voltage fraction of discharge was identical by definition because in neither case was the beam voltage measured successfully. A $10 \%$ error bound was used for $V_{d}$. Mass utilization efficiency in this case was quite low compared to his calculation of $97 \% \pm 12 \%$. However, this result was somewhat arbitrary since his calculations were resulting in nonsensical values. A low mass utilization efficiency is not surprising, however, because argon is roughly 3 times less massive than xenon, and $\eta_{m}$ is directly proportional to ion mass. Electrical efficiencies between the two cases were similar. His calculation resulted in an $\eta_{o}$ of $77 \% \pm 2 \%$ compared to $70.64 \%$ in this case. This again points to the conclusion that the thruster is less efficient when operated at higher discharge power. The total thrust correction factor was also lower than his result of $81.4 \%$ because of the higher calculated divergence angle. 
All of this resulted in a very low total efficiency of $10.63 \%$, roughly one third of his calculated $29 \%$. As mentioned previously, much of this result is likely attributable to the use of argon instead of Xenon and the operation at a higher power. Comparing to Scott's case is not a 1:1 comparison. Thrust was also significantly lower at roughly $60 \%$ of his calculated $20.4 \mathrm{mN}$. Specific impulse was determined to be 1360 s, much lower than the 2438 s predicted by his thrust and mass flow rate values. This is expected due to both a lower thrust and higher mass flow rate in this case.

\section{2}

\section{SHIELDED HEMPT RESULTS}

After data was collected in the unshielded configuration, the shield was placed on the HEMPT and the process was repeated. This test was performed between the first and second unshielded tests. Like the first unshielded run, data for this plume profile were collected using the Faraday probe. The data for this run are plotted in Figure 35.

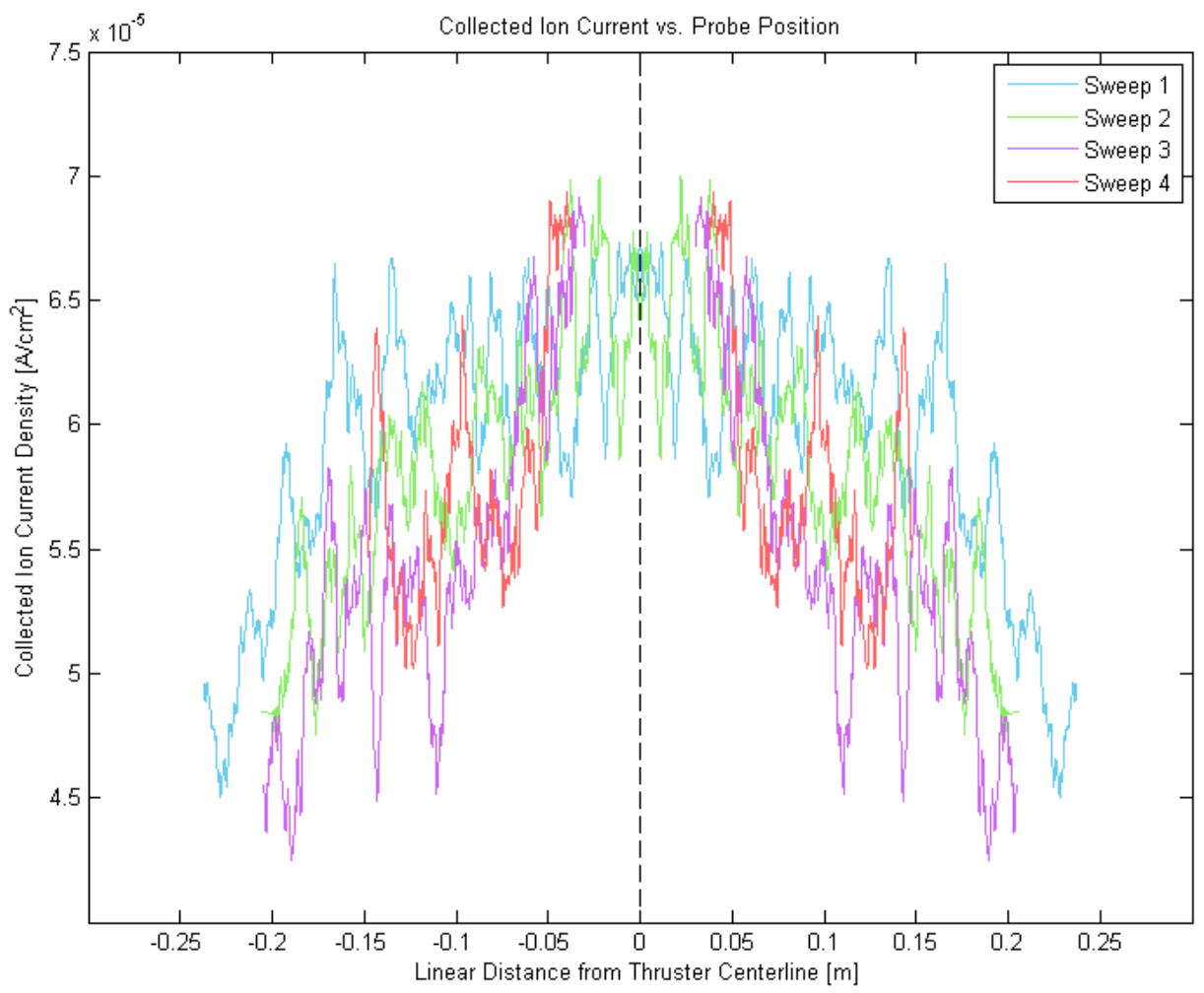

Figure 35. Shielded HEMPT Ion Current Plume Profile. 
As mentioned previously, these data were insufficient to predict the beam current because the high background pressure affected the nude Faraday probe's ability to get accurate current measurements. However, it is clear from Figure 35 that the applied shield had a significant impact on the hollow nature of the plume at the centerline. Compared to Figure 33, there is a peak at the centerline instead of a significant valley.

Note the data for Sweeps 3 and 4 are missing near the centerline. This is because the probe drive system, when commanded to sweep the entire length of the plume, got stuck at certain points and was unable to go further. Part of the problem may be that the rails of the probe drive system are exposed to the high energy ions of the plume, and they became rough in certain spots due to surface sputtering. At times these rough areas impeded the movement of the probes. The probe position versus time for this run is plotted in Figure 36. The colored portions of the plot correspond to the colors of the sweeps in Figure 35. The grayed out sections show where the probes stopped between sweeps. Data were not collected during these stall times.

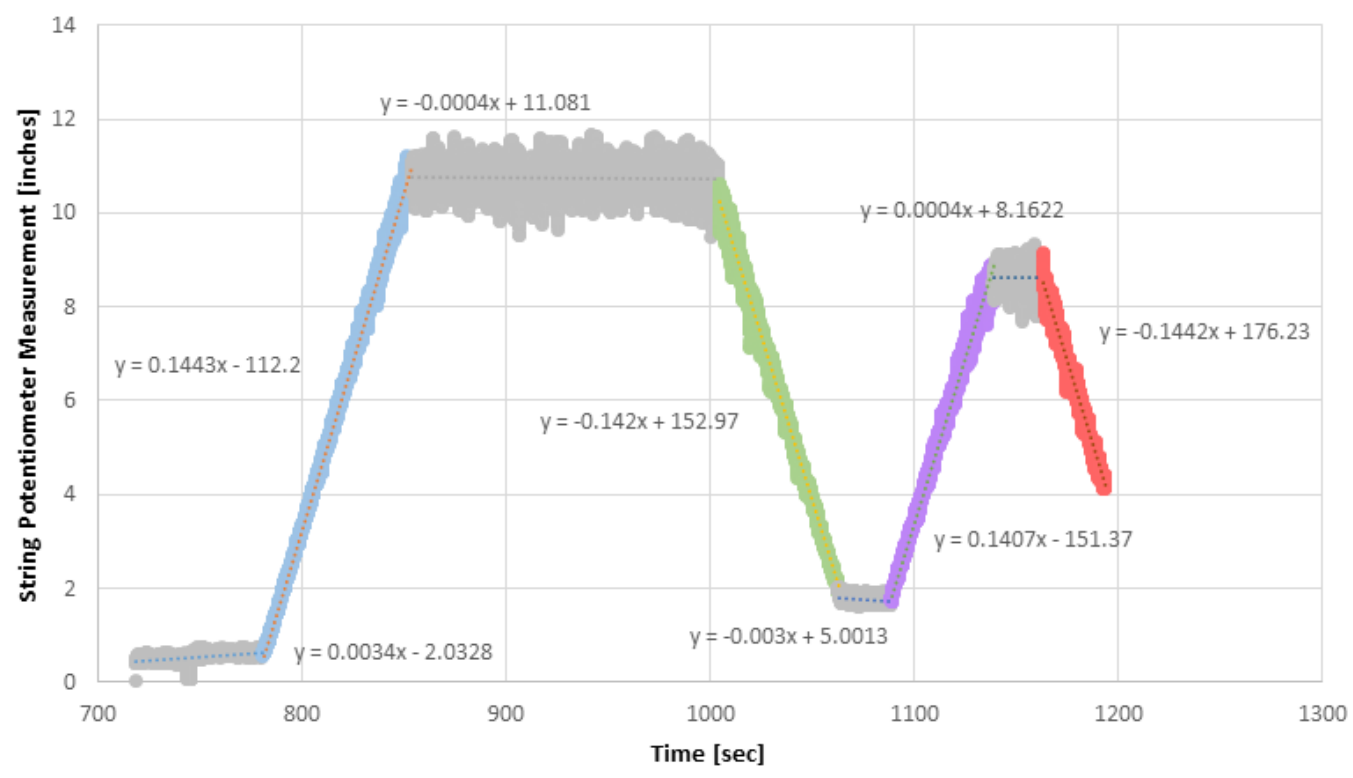

Figure 36. String Potentiometer Position Vs. Time for the Unshielded HEMPT.

An unexpected result of using the string pot for distance measurements was that it experiences more noise when extended. This is likely because the conductive string becomes 
more exposed to the plasma environment as it is extended further, allowing electrical noise to more easily couple to it. For this reason, a linear fit was applied to each region of the data and this fit was used as the actual location for the Faraday probe and RPA measurements. Previous experimentation showed the probe drive physically moving due to noise, but this was not the case for this experiment. The chamber view port allowed for the probe position to be verified, and it was clear that the noise in Figure 34 was only electrical noise and did not result in any probe motion.

Unfortunately, the HEMPT experienced catastrophic overheating after the unshielded test with the RPA as a Faraday probe. This meant that it was unable to run in the shielded configuration again to get a better shielded ion current profile. Therefore, it is difficult to make meaningful conclusions about the effects of the shield on the thruster's plume and performance. What is known is that the shield seems to provide an increase in ion current at the centerline compared to the unshielded thruster. Whether or not this results in an increase in performance is still in question.

The results of all 4 tests are summarized in Table 14.

Table 14. Summary of HEMPT Test Results.

\begin{tabular}{|c|c|c|c|c|}
\hline & $\begin{array}{c}\text { Test } 1 \\
\text { Unshielded, } \\
\text { Faraday Probe }\end{array}$ & $\begin{array}{c}\text { Test } 2 \\
\text { Shielded, } \\
\text { Faraday Probe }\end{array}$ & $\begin{array}{c}\text { Test } 3 \\
\text { Unshielded, RPA } \\
\text { as Faraday Probe }\end{array}$ & $\begin{array}{c}\text { Test } 4 \\
\text { Shielded, RPA } \\
\text { as Faraday } \\
\text { Probe }\end{array}$ \\
\hline $\begin{array}{r}\text { Total Efficiency } \\
\text { Thrust }\end{array}$ & $\begin{array}{c}\text { Data Unusable- } \\
\text { Faraday Probe }\end{array}$ & $\begin{array}{c}\text { Data Unusable } \\
\text { - Faraday }\end{array}$ & $\begin{array}{l}10.63 \% \pm 3.66 \% \\
12.1 \mathrm{mN} \pm 1.3 \mathrm{mN}\end{array}$ & \\
\hline Specific Impulse & & & & $\begin{array}{l}\text { No Data- } \\
\text { Thruster }\end{array}$ \\
\hline Summary & $\begin{array}{l}\text { Visible dip in } \\
\text { centerline } \\
\text { current }\end{array}$ & $\begin{array}{l}\text { Visible peak in } \\
\text { centerline } \\
\text { current }\end{array}$ & $\begin{array}{l}\text { Visible dip in } \\
\text { centerline current }\end{array}$ & Damaged \\
\hline
\end{tabular}

Tests 1 and 2 were useful in showing that the addition of the shield seems to increase centerline current, which was the predicted result. However, the data was not able to be used in the calculation of thruster performance parameters, as previously mentioned. Test 3 resulted in 
successful characterization of the unshielded HEMPT, with a very significant collected ion current local minimum at the centerline. Test 4 was unsuccessful due to thruster failure. 


\section{CONCLUSION}

The goal of this thesis at its outset was to characterize the magnetic field of the laboratory HEMPT, determine a shield design to improve this field, and measure the performance of the thruster in a vacuum with and without the shield. The goal of the shield was to reduce the divergence half angle of the ion beam. The field was characterized and modeled using COMSOL and the model was verified by direct measurement. A shield design was determined which would theoretically improve the magnetic field. The performance of the thruster was characterized in the unshielded configuration, but not the shielded configuration. However, preliminary measurements of the shielded configuration show an increase in centerline current, indicating a decrease in divergence half angle. Further testing will need to be performed to fully characterize the shielded HEMPT.

The unshielded HEMPT produced $12.1 \pm 1.3 \mathrm{mN}$ of thrust with a specific impulse of $1361 \pm 147 \mathrm{~s}$. The thruster operated with a total efficiency of $10.63 \pm 3.66 \%$, an efficiency much lower than expected. A large contributor to this low efficiency is the use of argon in place of xenon. Its lower mass and higher ionization energy make it a less efficient propellant choice.

Further, the thruster is prone to overheating, indicating that significant thermal losses are present in this design. A cathode voltage that was $33 \%$ higher than expected is also a source of efficiency loss. 


\section{BIBLIOGRAPHY}

[1] D. Goebel and I. Katz, Fundamentals of Electric Propulsion: Ion and Hall Thrusters, John Wiley \& Sons, Inc., 2008.

[2] "SSME," Encyclopedia Astronautica, [Online]. Available:

http://www.astronautix.com/engines/ssme.htm. [Accessed 145 2015].

[3] G. P. Sutton and O. Biblarz, Rocket Propulsion Elements, Wiley, 2010.

[4] G. Kornfeld, N. Koch and G. Coustou, "The HEMP Thruster: An Alternative to Conventional Ion Sources?," 2003.

[5] G. Kornfeld, N. Koch and H.-P. Harmann, "Physics and Evolution of HEMPT-Thrusters," in International Electric Propulsion Conference., Florence, Italy, 2007.

[6] G. Kornfeld, N. Koch and G. Coutou, "First Test Results of the HEMP Thruster Concept," Thales Electron Devices , 2003.

[7] D. Goebel, Personal Correspondence, 2015.

[8] W. D. Callister and D. G. Rethwisch, Materials Science and Engineering, 8th Edition, Wiley.

[9] W. F. Brown, Handbook of Chemistry and Physics, Ch. 8, Magnetic Materials, McGrawHills, 1958.

[10] J. Pyrhonen, T. Jokinen and V. Hrabovcova, Design of Rotating Electrical Machines, John Wiley and Sons, 2009.

[11] Y. Raitses, J. C. Gayoso and N. J. Fisch, "Effect of Magnetic Shielding on Plasma Plume of the Cylindrical Hall Thrusters," in International Electric Propulsion Conference, 2011.

[12] PEPL, "Retarding Potential Analyzer (RPA)," The University of Michigan Department of Aerospace Engineering, 12 July 2010. [Online]. Available: http://pepl.engin.umich.edu/diagnostics/RPA.html. [Accessed 7 July 2015].

[13] PEPL, "Faraday Probe," The University of Michigan Department of Aerospace Engineering, 6 July 2010. [Online]. Available: http://pepl.engin.umich.edu/diagnostics/Faraday.html. [Accessed 7 July 2015].

[14] M. L. R. Walker, R. R. Hofer and A. D. Gallimore, "The Effects of Nude Faraday Probe Design and Vacuum Facility Backpressure on the Measured Ion Current Density Profile of Hall Thruster Plumes," in AIAA/ASME/SAE/ASEE Joint Propulsion Conference \& Exhibit, Indianapolis, 2002.

[15] S. A. McGrail, Hollow Plume Mitigation of High-Efficiency Multistage Plasma Thruster, San Luis Obispo: California Polytechnic State University, 2013.

[16] D. Bruno, C. Catalfamo, A. Laricchiuta, D. Giordano and M. Capitelli, "Convergence of CHapman-Enskog Calculation of Transport Coefficients of Magnetized Argon Plasma," Physics of Plasmas, vol. 13, no. 7, 2006.

[17] D. W. Knapp, "Implementation of a 1/4 inch Hollow Cathode into a MIniature Xenon Ion Thruster (MiXI)," California Polytechnic State University, San Luis Obispo, 2012.

[18] MKS Instruments, Granville-Phillips Series 274 Bayard-Alpert Type Ionization Gauges Instruction Manual - Rev B, 2014.

[19] G. Parissenti, N. Koch, D. Pavarin, E. Ahedo, K. Katsonis, Scortecci, F. and M. Pessana, "Non Conventional Propellants for Electric Propulsion Applications," Space Propulsion 1841086, 2010.

[20] OMEGA, "FMA A2300 Massflow Meter User's Guide," [Online]. Available: http://www.omega.com/Manuals/manualpdf/M2842.pdf. [Accessed 8 July 2015]. 
[21] R. R. Hofer, Development and Characterization of High-Efficiency, High Specific Impulse Xenon Hall Thrusters., University of Michigan, 2004.

[22] M. L. R. Walker, A. L. Victor, R. R. Hofer and A. D. Gallimore, "Effect of Backpressure on Ion Current Density Measurements in Hall Thruster Plumes," Journal of Propulsion and Power, vol. 21, no. 3, pp. 408-415, 2005.

[23] K. K. Jameson, Investigation of Hollow Cathode Effects on Total Thruster Efficiency in a 6kW Hall Thruster, Los Angeles: UCLA, 2008. 


\section{APPENDICES}

\section{A. LESSONS LEARNED AND RECOMMENDATIONS}

This thesis provided an innumerable number of lessons learned. Or, more blatantly stated, that means there were a lot of problems. Some of the most important ones, as well as future steps, are explained below.

One important way in which this thesis improved on previous work was through direct measurement of the thruster's magnetic field to verify a magnetic model. While the measurements that were collected are acceptable there is still room for improvement. A more robust measurement apparatus should be constructed to get more exact measurements. The basic idea is a magnetic probe mount with 2-axis control. It must be able to know fairly precisely (within maybe 10 thousandths of an inch or less) where the probe tip is and it must keep the thruster stationary. The material of the mount must be non-magnetic so that the probe readings aren't affected. The string potentiometer, or perhaps two, could be used to get this level of accuracy.

Get the chamber functioning early on. Don't assume it will work when it needs to. There is plenty that can stop it from getting down to the required pressure. One of the most annoying causes of this is leaks. Use the leak detector if necessary (see the process in the appendix). The Orings are a likely place for leaks to occur (clean surfaces and o-rings before installing). Also, the electrical feedthroughs are quite leaky. Even if not in use, connect a coaxial cable to them to significantly decrease the leak rate. The chamber could not reach pressure for this thesis until this was done.

Another recommendation is to not underestimate setup time. Think about how long it will realistically take to setup the experiment and then multiply by a factor of at least 3 . EP electrical setups are necessarily complex, so there are many connections which must be properly made. Lines must be electrically shielded from the plasma. The DAQ inputs must be scaled using properly sized resistors. Capacitors must be placed strategically to decrease noise. Further, all 
electrical components must be sized properly so that they don't see too much power. It's very easy to blow out a resistor or capacitor if this isn't taken into account. Collecting enough power supplies and wiring them properly can also pose a challenge. Double check all connections, and then check them again. Also account for things to go wrong. In this thesis, perhaps the worst perpetrator of this was the chamber's hoist mechanism, which failed. This meant a forklift was required to open and close the chamber. This slowed down testing considerably, as it meant changes could only be made when Cody was around to operate the forklift.

Setting up a program to get data from the DAQ was also time consuming. Future work on this thesis should probably use the LabVIEW program written here to decrease that time. A big part of this is understanding the limits of the DAQ. For example, it can't accept just any voltage. There are minimum and maximum acceptable voltages as well as minimum step sizes within that range. Proper voltage divider design is important to ensure that incoming signals aren't too large or too small for the DAQ to handle.

Expect the unexpected. It's truly incredible how many things went wrong with the vacuum chamber, the facilities, the electronics, and basically every other aspect of this thesis. The mech pump stopped working, the compressor leaked all of its helium overnight, the cathode had issues lighting, the flow meters were 5 years past their calibration dates and had to be sent out for 6 weeks to be calibrated, the chamber had numerous, elusive leaks, and so much more. Many hours were spent on troubleshooting, problem solving, and often waiting for parts to be delivered. Testing itself takes a very short time. All of this meant this thesis took about 5 months longer than planned. However, much of this can be remedied by starting work on the project as early as possible.

The RPA needs to be opened up and fixed so that it works reliably. Grid 3 must be replaced because of the small hole that is currently present. There is no reason why it shouldn't work. The physics of operation are fairly straight forward; it's a matter of putting it into practice. Make sure that the connections from the power supplies to the grids are good. After struggling 
with getting it to work, it was discovered that a crimp connection had failed. After fixing this and trying again, the hole in Grid 3 was found. It was frustrating not being able to use the probe for its intended purpose for the duration of this thesis, especially after Scott experienced a different issue with the same result. To whoever continues this work, don't let that happen again!

Another improvement is to use xenon in place of argon if finances allow. Argon is exponentially cheaper, but Xenon would have allowed direct comparison to Scott's results and likely would have permitted the cathode to behave properly. The high flow rate of argon needed to keep the cathode lit caused a sharp increase in chamber pressure which made the Faraday probe close to useless. Perhaps using argon initially to test the setup and make sure everything is in order is a good idea, but then switch to Xenon to actually take data.

It's also probably worth investigating why the cathode was pulling additional power. It may have been a result of using argon instead of xenon or it may be due to the cathode placement relative to the thruster. This can be investigated by placing a steel plate as an anode directly in front of the cathode and running it in this configuration. If the cathode runs normally, then it's something with the cathode location relative to the thruster causing the problem. If it doesn't, perhaps it's some wiring/connection issue or the cathode needs maintenance.

The cathode is very sensitive to air when it's hot. Because of this, it's important to ensure that the lines are free of air. This means pumping the lines out with the chamber. The gas lines are a common source of leaks, so getting the gas feed system airtight can take up a lot of time but it's necessary to protect the cathode.

The probe drive design should probably be revisited. A radial probe system would be more desirable. If a linear drive is still desired, a better design should be considered. The current one is not very reliable due to the belt slipping, the rails impeding the probes, and the probe's wires getting caught on the structure. Also, the carbon sheeting which covers the top of the chamber was prone to sag and block the probe drive from moving. It's important to verify the probes can travel the length of the track before pumping down each time. 
A big improvement to this thesis could be made by using (or creating) a software package which allows for the optimization of shield designs. Essentially, several desirable characteristics about the field (non-radial at exit, minimal internal strength change, etc.) have been identified. A program or suite of programs which could iterate through various shield geometries and output the best result would be very useful.

Perhaps the most important improvement that must be made to this experiment is with the HEMPT itself. The device should probably be rebuilt from the ground up. Many of the necessary specialty parts can be found in the box of HEMPT equipment. First, thermal modelling and mitigation should be performed so that the Mk. II design doesn't experience overheating like the model used in this thesis. This may mean using more heat resistant magnets, better heat sinks, or just some change to the design that isolates the magnets from the extreme heat of the thruster. In any case, several thermocouples should be placed strategically on the HEMPT and it should be shut down whenever a certain threshold is reached. The temperature was not monitored in this thesis and that was a mistake. 


\section{B. PROCEDURES}

There are two procedures for bringing the HVEC to the necessary pressure to begin testing. The first is the preferred method, as it clears previously condensed gasses from the cryo pump, essentially regenerating the cryo pump. The second allows for shorter pump down times but does not regenerate the cryo pump. The first procedure should be performed at least every few runs to clear the cryo pump, or whenever the cryopump is taking abnormally long to bring the chamber down to pressure.

\section{HVEC CHAMBER OPERATION, CRYO PUMP WARM}

1. Turn on the facility compressed air supply via the valve on the wall.

2. Turn on System Power switch on chamber front panel.

3. Turn on Mechanical Pump.

4. Turn on Chamber Rough.

5. Allow chamber to pump down to 50-100 mTorr

6. Turn on Pressure Override.

7. Turn on Rough Interlock.

8. Open the Gate Valve. Pressure will rise as gas previously condensed in the cryo pump is released.

9. Allow chamber to pump down to 50-100mTorr again.

10. Turn on the Compressor.

11. Allow the cryo pump temperature (displayed on front panel) to reach $150 \mathrm{~K}$ or lower.

12. Turn off the Mechanical Pump.

13. Turn off Rough Interlock.

14. Turn on Pressure Interlock.

15. Turn off Pressure Override. 
16. Allow cryo pump temperature to fall to approximately $17 \mathrm{~K}$. Do not operate the cryo pump with the gate valve open if pressure is above 150 mTorr.

17. When the convectron gauge reads 0 (shortly after reading $1 \times 10^{-4}$ Torr) turn on the ion gauge using the $I G l$ switch on the pressure sensor controller.

18. Allow chamber to reach a pressure of $5 \mu$ Torr or lower before heating the cathode.

\section{HVEC CHAMBER OPERATION, CRYO PUMP PRECOOLED}

1. Turn on Mechanical Pump.

2. Turn on Chamber Rough.

3. Allow chamber pressure to reach 50-100mTorr.

4. Close Chamber Rough.

5. Turn off Mechanical Pump.

6. Turn on Pressure Interlock.

7. Open Gate Valve. Pressure will drop substantially.

8. When the convectron gauge reads 0 (shortly after reading $1 \times 10^{-4}$ Torr) turn on the ion gauge using the $I G 1$ switch on the pressure sensor controller.

9. Allow chamber to reach a pressure of $5 \mu$ Torr or lower before heating the cathode.

\section{HVEC CHAMBER SHUT DOWN AND VENT}

1. Turn off the Ion Gauge using IGl button.

2. Shut the Gate Valve.

3. Turn off Pressure Interlock.

4. Turn off the Compressor.

5. Open the Vent Valve.

6. Allow the chamber to reach ambient pressure (760 Torr)

7. Close the Vent Valve. 
8. Close the compressed air supply at the wall.

9. Turn off System Power.

\section{HOLLOW CATHODE OPERATION}

1. Mount the cathode in the vacuum chamber in the desired location

2. Connect the cathode common node (Point 1 in Figure 37) to common ground.

3. Connect the heater supply to the heater connection node (Point 2).

4. Connect the propellant feed line to the cathode (Point 3).

5. Connect the Keeper supply to the keeper screw (Point 6).

Note: Points 4, 5, and the screw by 6 are there to keep the hollow cathode assembly together. They are electrically connected to the chassis and electrically isolated from the cathode base.

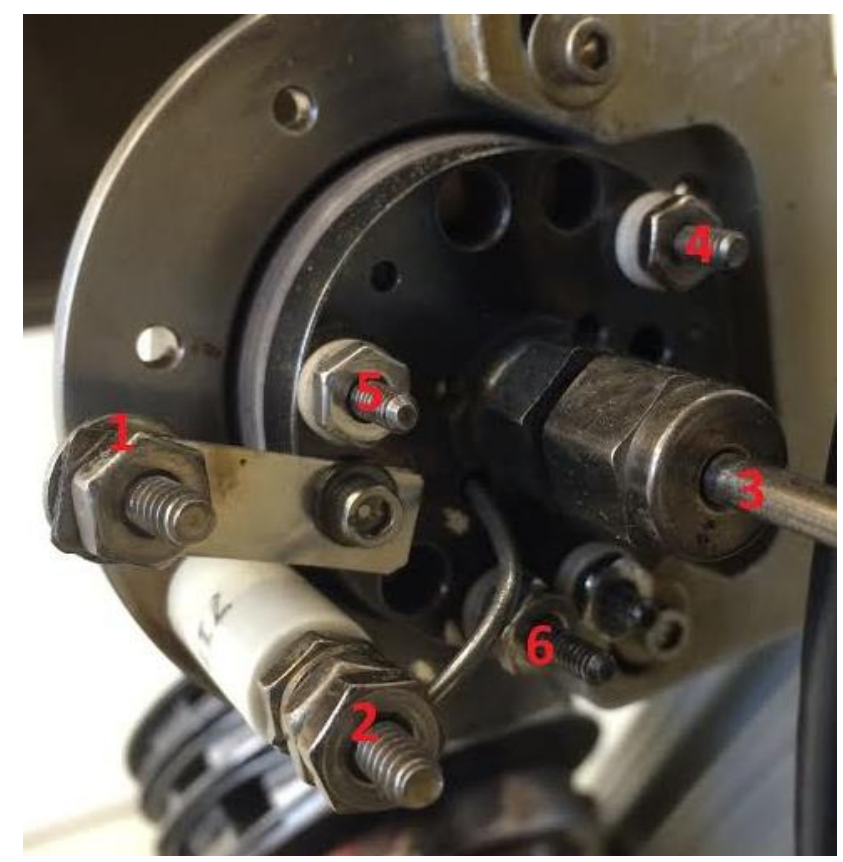

\section{Figure 37. Hollow Cathode Electrical Connection Points.}

6. Close the chamber.

7. Open all propellant lines to the vacuum chamber. 
8. Use one of the previous procedures to pump the chamber down to $5 \mu$ Torr or lower.

9. Turn on heater supply to $6 \mathrm{~A}$ for 20 minutes.

10. Turn on the gas supply (use only ultra high purity xenon or argon)

11. Set the stainless steel regulator on the propellant tank to 25 psi.

12. Adjust the cathode's needle valve so $5 \mathrm{sccm}$ of gas is going to the cathode. Note that the flow meter is calibrated for nitrogen, so a conversion factor must be used to obtain the true flow rate for the gas being used.

13. Light the cathode. The cathode is lit when the keeper supply begins pulling current.

a. Turn on the keeper supply and slowly increase the voltage. The cathode can theoretically light at around $120 \mathrm{~V}$, but in practice the necessary voltage is often much higher.

b. If the keeper supply maxes out (314V) and the cathode is still not lit, turn on the igniter supply and slowly increase its voltage. Ensure both supplies are properly protected with high voltage diodes. The cathode should light with less than $1 \mathrm{kV}$. When it does, turn off the igniter supply. Set the keeper supply's current to 2A.

14. Turn off the heater supply.

15. Allow cathode to run in self-heating mode for required test duration.

16. When done, turn off all power supplies and stop gas flow to the cathode.

17. Allow cathode to cool for at least 2 hours prior to breaking vacuum.

\section{THRUSTER OPERATION}

1. Setup the Cathode per the above procedure.

2. Secrely mount the thruster in the desired orientation in the chamber.

3. Attach the propellant flow line to the back of the thruster. 
4. Attach the positive lead of the discharge supply to the copper connector on the HEMPT's propellant line.

5. Pump down the chamber per one of the above procedures to reach a pressure of $5 \mu$ Torr or lower. Ensure the propellant lines are open so air is evacuated from them as well.

6. Light the hollow cathode per the above procedure, but also open the shut off valve to the thruster and adjust gas flow to $5 \mathrm{sccm}$ (actual flow, not what's displayed by the flow meter) at the same time as cathode flow is initiated.

7. Once the cathode is lit, turn the voltage on the discharge supply up to $200 \mathrm{~V}$.

8. Adjust the discharge supply voltage to the desired value based on testing needs.

9. Perform desired tests.

10. Shut off all power supplies and gas flow to the system.

11. Let the cathode and thruster cool for at least 2 hours prior to breaking vacuum.

\section{CRYO PUMP DECONTAMINATION}

This procedure describes the process for purging the cryo pump helium circuit of air or other contaminants. It should be followed if the pump, lines, or compressor's helium store is suspected to be contaminated. This procedure MUST be followed if at any point the compressor's supply pressure reads 0 psi. This procedure will consume significant amounts of helium gas, so ensure the helium tank has more than 1000psi or so prior to starting.

1. Ensure compressor is turned off, as well as other machines in the lab. The quieter the lab is the easier it will be to locate leaks.

2. Refill the compressor with 50 psi of UHP helium (see the compressor manual for how to add helium). 
3. Listen for helium leaking from the compressor, lines, and pump (henceforth the helium circuit). Particularly, check the self-sealing connections where the lines attach to the pump and compressor.

4. Remedy any leaks until no leaks can be heard and the compressor's pressure holds steady.

5. Refill the compressor with 200 psi of UHP helium.

6. Disconnect the supply and return lines from the pump, but leave them attached to the compressor.

7. Connect the disconnected supply and return lines to the purge manifold, shown in Figure 38.

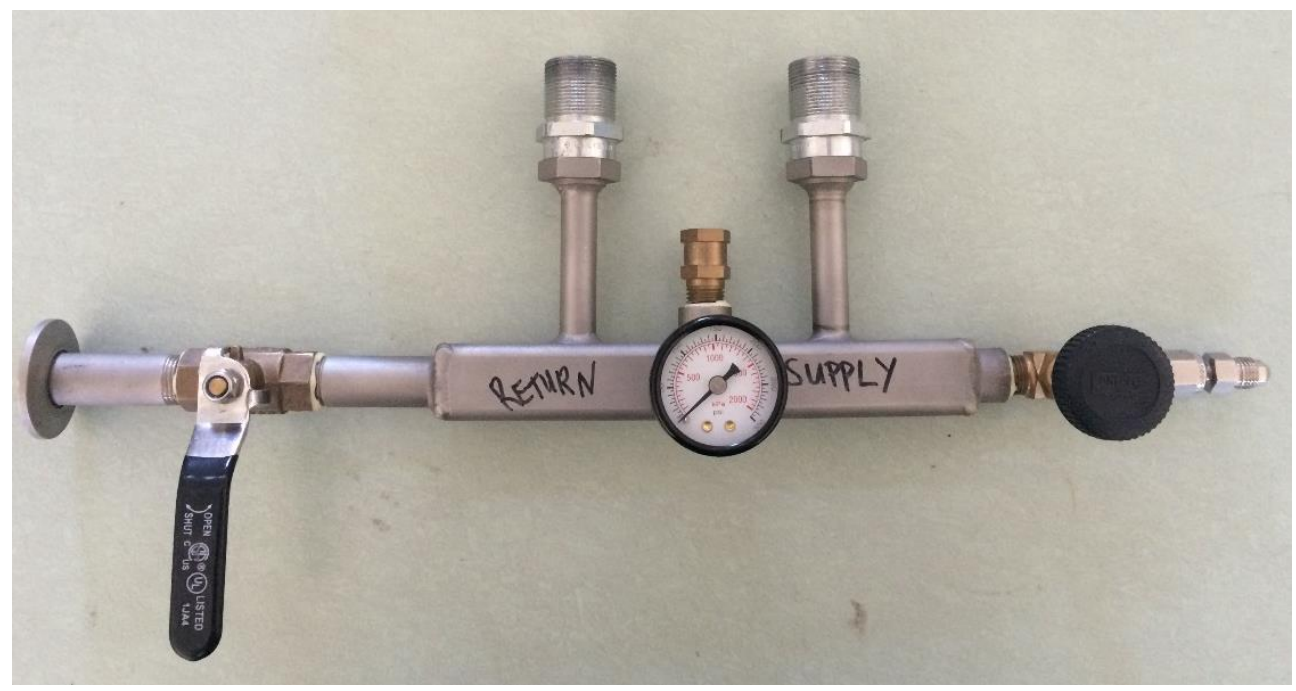

Figure 38. Helium Purge Manifold.

8. Connect the helium tank to the swagelok valve on the right side of the manifold in Figure 38. Make sure to run helium through the line at a low psi to clear the line of air as you connect it to the mainfold.

9. Open the swagelok valve.

10. Set the helium tank regulator to $200 \mathrm{psi}$.

11. Close the swagelok valve. 
12. SLOWLY open the vent valve on the left side of the manifold in Figure 38 until the pressure gauge on the manifold reads about 50 psi. This will bleed helium from the lines and compressor unit. It's important not to bleed all of the pressure as this will recontaminate the helium circuit.

13. Close the vent valve on the manifold.

14. Repeat steps 9-13 five times to ensure the helium in the compressor and lines is at an acceptable level of purity.

15. Open the swagelok valve one final time to fill the compressor and lines with 200 psi of helium.

16. Disconnect the return and supply lines from the purge manifold.

17. Reconnect the return and supply lines to the pump.

18. Run the compressor and cold head normally until the temperature indicator shows $17 \mathrm{~K}$ or a ratcheting noise is heard. The system should be monitored for the duration of the cooling period. A ratcheting noise indicates that significant amounts of contaminants are freezing in the cold head, increasing the mechanical force needed to run the pump. Proceed immediately to the next step if ratcheting is audible.

19. Disconnect the supply line from the compressor.

20. Once the supply line is disconnected, disconnect the return line from the compressor.

21. Turn off the cold head and compressor. The condensible contaminats are now isolated in the cold head.

22. Allow cold head to warm to room temperature.

23. Connect the supply and return lines to the purge manifold.

24. Using the same procedure from steps 9-15, purge the cold head and helium lines. Run the cold head in between purges to further clear contaminants. 
25. Verify the cold head pressure held through the cycles (via the manifold's pressure gauge.)

At this point, the entire helium circuit should be decontaminated.

26. Disconnect supply and return lines from the purge manifold.

27. Reattach supply and return lines to the compressor.

28. Run the system normally and listen carefully for ratcheting or other anomalies. If ratcheting occurs, this procedure will have to be repeated because the helium circuit was somehow not completely decontaminated.

\section{HELIUM LEAK DETECTOR OPERATION}

The BMED Engineering department has a helium leak detector device which they graciously lent out for this project. The basic procedure for its use involves using it to pump down the chamber and then spraying potential leak points with small amounts of helium. If the leak detector responds to the spray, there is a leak there. If no response, there probably isn't a leak there. This device proved invaluable in solving leak problems which were preventing the chamber from reaching the stringent pressure requirement of $5 \mu$ Torr. The electrical feedthroughs on this chamber are a significant source of leaks. These alone are sufficient to prevent the chamber from reaching the necessary pressure. The easiest fix for this is to screw coaxial cables with the appropriate connectors onto the feedthroughs.

1. Contact Dr. Richard Savage or Dave Laiho for permission to use the leak detector.

2. Ensure the chamber is at ambient pressure.

3. Locate the manual vent port on the back of the chamber. 
4. Connect the leak detector's flex hose to the manual vent port on the back of the chamber as shown in Figure 39.

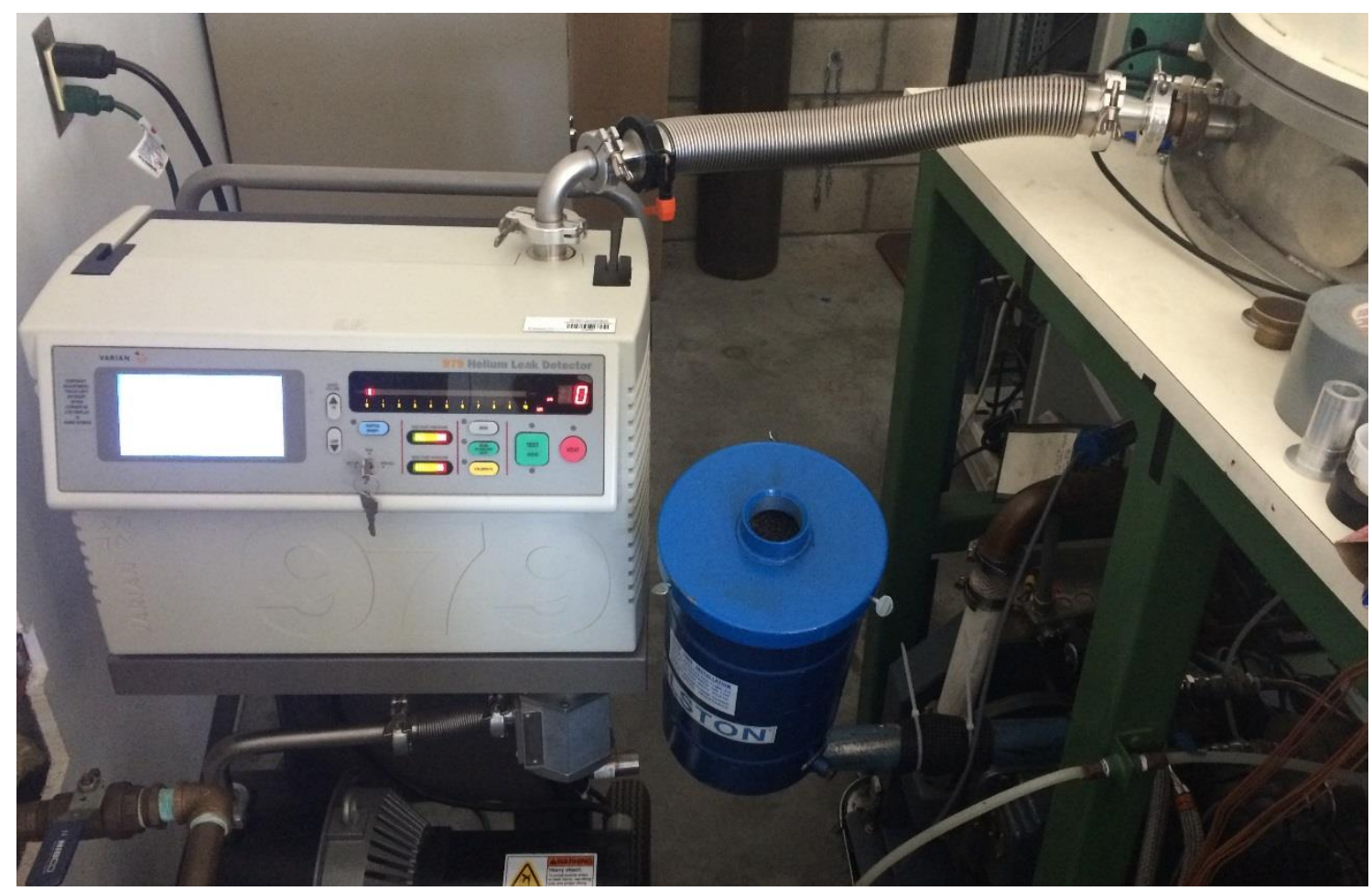

Figure 39. Leak Detector Connected to Vacuum Chamber.

5. Remove the manual vent port by hand-turning it counterclockwise until the end cap can be removed by pulling it out. See Figure 40 for the names and images of the parts needed for this procedure.

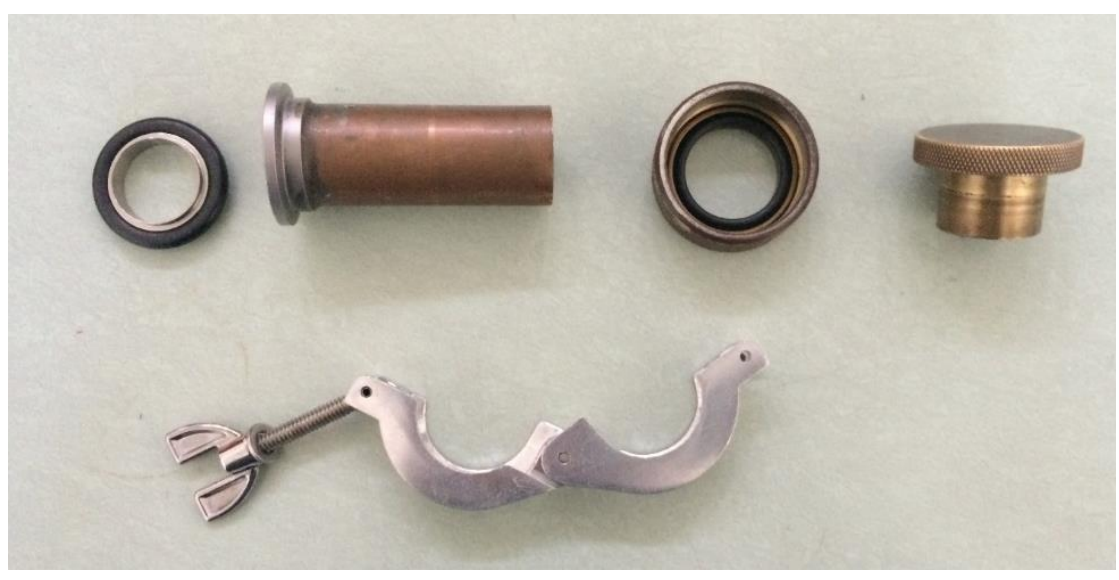

Figure 40. Components Needed for Connecting Leak Detector to Chamber. From upper left to bottom: O-ring, Chamber Port Flange Insert, Brass Backing, End Cap, O-ring Clamp. 
6. Replace the end cap with the chamber port flange insert into the brass backing where the end cap of the manual vent port was.

7. Hand tighten the brass backing with the chamber port flange inserted.

8. Line up the tube from the Varian leak detector with the chamber port flange insert and place the o-ring between the hose and the insert.

9. Fasten the o-ring clamp around this connection to secure it in place.

10. Ensure the chamber is closed.

11. Turn on the Varian leak detector using the power switch on the rear of the device.

12. Wait for the device to finish its start-up sequence, then press "Test".

13. The leak detector with now pull a vacuum on the chamber. Wait for "Test Port Pressure" and "Spec Tube Pressure" readings to fall to at least the orange colored range. This indicates the leak detector is ready to detect leaks. The screen should read "Fine Test".

14. Connect a flow regulator to the helium tank.

15. Connect a mesh flex tube to the other end of the flow regulator.

16. Attach a spray nozzle to the other end of the mesh flex tube, shown in Figure 41.

17. Pressurize the helium line with the lowest psi possible.

18. Place the spray nozzle near areas of suspected leaks and depress the handle a small amount. Start from the top of the chamber and work down, as helium rises

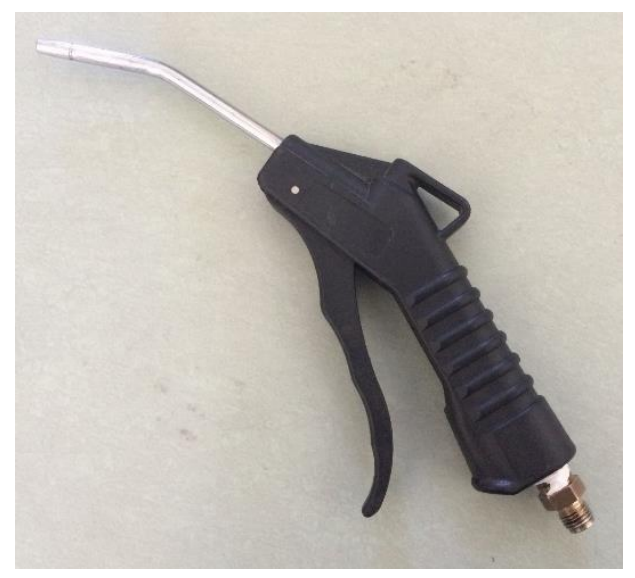

Figure 41. Spray Nozzle. 
in the atmosphere.

19. Wait at least 10 seconds to see if the leak detector's leak rate indicator shows an increase in leak rate. If a significant leak rate increase is observed, there is likely a leak near that location.

20. If the leak detector's leak rate does not return to the low level it originally detected, the device may be saturated with helium. In this case, the fastest way to reset it is to use the device to vent the chamber and then re-pump it down with the device. This should reset the leak rate.

21. It is often difficult to pinpoint the leak after you know the general area it's in. This is because the chamber has many feed throughs and other possible leaks which are close to each other. So the best method is to use a plastic bag or other container along with tape to enclose the suspected area. Plastic cups work well for this, as shown in Figure 42.

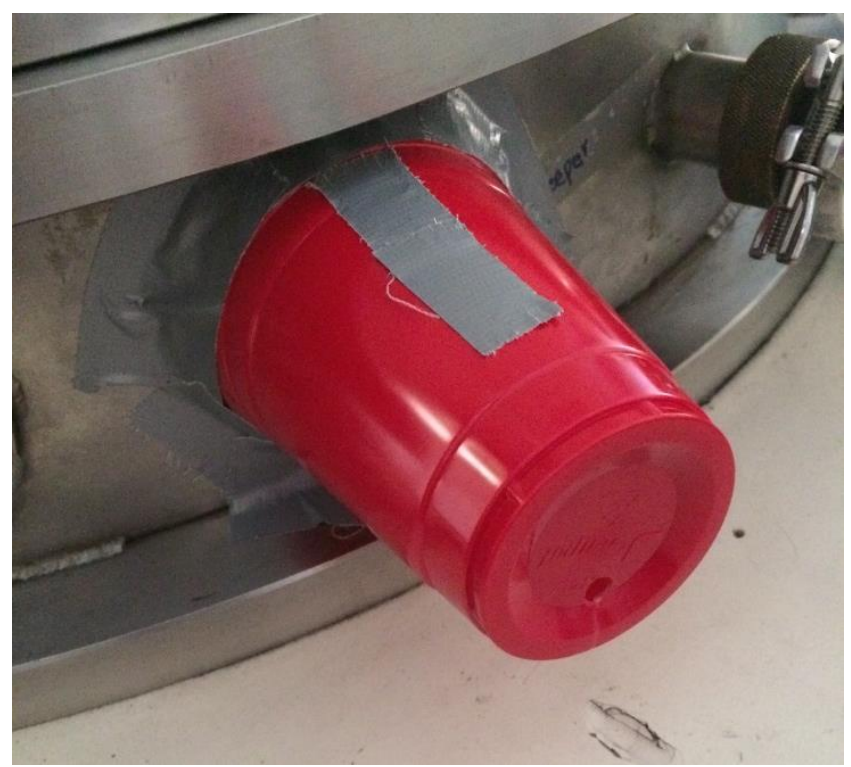

Figure 42. Improvised Helium Enclosure Over Area of Suspected Leak. A hole is placed at the bottom of the cup to provide a means of filling the cup with helium. 
22. Test the o-ring seals, electrical feedthroughs, window, top of the chamber, etc. for leaks. The o-rings and electrical feedthroughs particularly are sources of leaks. O-rings can be cleaned or replaced. The electrical feedthroughs should be "capped" by tightly screwing cables with the appropriate connectors on to them.

23. Fix any found leaks and try pumping the chamber down to the desired pressure again.

24. Repeat this procedure as necessary. 


\section{WALL EROSION REDUCTION EFFICACY}

The HEMPT geometry is extremely good at minimizing wall erosion from plasma impact. During experimentation, graphite sheeting was placed above the HEMPT's ion beam to prevent damage to the chamber. This had the unexpected result of liberating some of the graphite from the sheets, which then deposited on the thruster below, including along the walls of the ionization chamber. The alumina, which is normally a bright white hue, was coated almost completely in the dark-grey graphite, shown in Figure 43. Note this image was taken after sanding the alumina to remove some of the graphite. The alumina, including the exit face, was initially coated almost entirely in grey. Note the 3 distinct white rings in the thruster. These are regions where the magnetic field is completely radial, allowing plasma to impact the walls. The area between the rings still has a dark layer of graphite, meaning little to no plasma impact was permitted. This is in sharp contrast to hall thrusters, which experience significant lifetime limitations due to wall erosion.

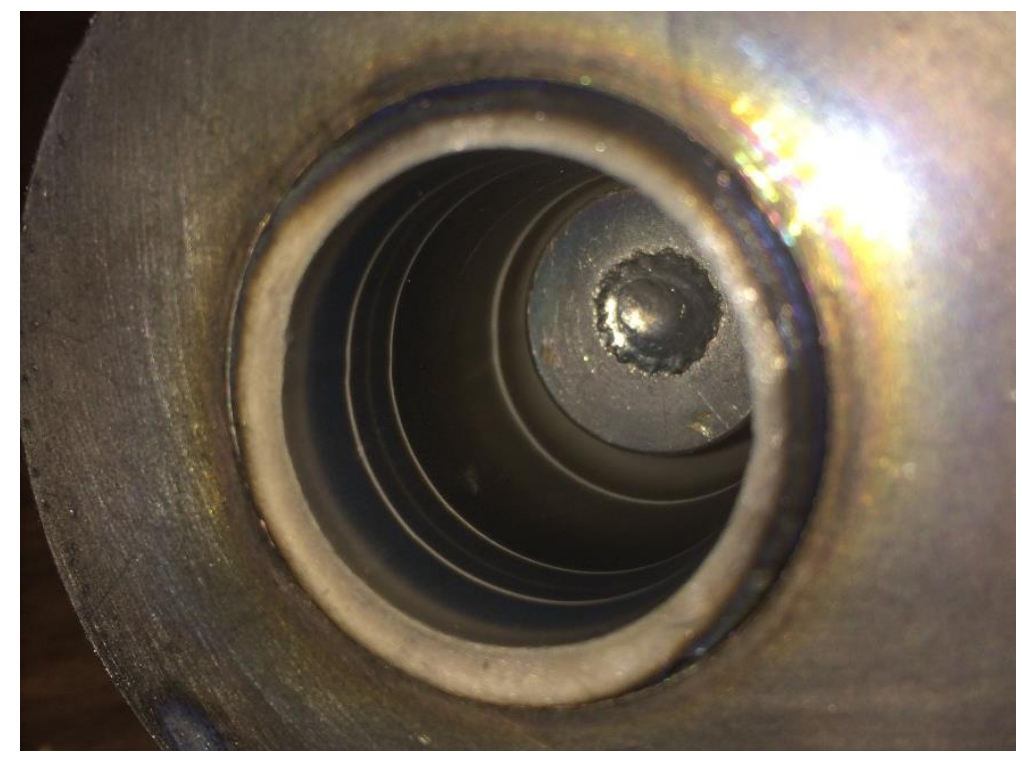

Figure 43. HEMPT Magnetic Cusp Lines. 


\section{LABVIEW CODE}

The LabVIEW backend consisted of two main while loops. The first will be referred to as the main loop, and the second is the keeper the loop. The main loop was responsible for reading data from the DAQ, filtering it, converting raw DAQ voltages to meaningful units, displaying data to the front panel, and recording the data in a log file. It also supported the input of a comment for the data file to help differentiate between data files. The keeper loop was used to query the keeper power supply. It was responsible for initializing the supply, querying the voltage and current, receiving a response, displaying the response, converting this to the dynamic data type (for better organized data collection) and writing the data to a file.

Two parallel loops were used because the keeper supply could not be queried as quickly as the DAQ data could be collected. This would cause the data collection speed to be limited by the keeper supply if everything was done in a single loop. With two loops, the DAQ data could be processed rapidly and the slower keeper data could be collected at its own slower frequency. The keeper loop's Write To Measurement File VI was activated with the same "Record State" button as the main loop via a local variable, so signals did not have to pass between the loops (which could result in a timing issue). Both loops are shown in Figure 45 and Figure 44. A case structure is included around the Keeper loop in case data collection is desired but the keeper supply is not on. Otherwise, the VI will error because it can't find the keeper supply.

Express VI's were used as much as possible to reduce programming time and increase ease of use. The DAQ Assistant reads all of the signals from the DAQ and provides a diagram of where to connect wires on the DAQ to read various signals. The Filter VI was used in moving average mode to smooth the incoming data from the DAQ. This was to reduce noise and provide cleaner, averaged data. Without this, much of the data (and especially the stringpot) tends to jump around wildly. A 25 point moving average was used. Also, it's worth noting that data collection was performed at a rate of $1 \mathrm{kHz}$, so averaging the last 25 values still allowed ample room for variation. The moving average was not so strong that it washed out the variability in the signal 
completely. After filtering, the signals were split into their individual constituents. The DAQ Assistant provided scaling for most values, but this wasn't possible for the pressure gauges. Both the convectron and ion gauge needed to be scaled based on a non-linear/non-polynomial formula, which the DAQ assistant could not handle. This is because the two gauges provide voltage from 0-10 volts with each voltage increment representing a new pressure decade. So, for example, the ion gauge reading zero volts meant a pressure of $1 \times 10^{-11}$, while a reading of 1 volt corresponds to a pressure of $1 \times 10^{-10}$, etc. The formula for this scaling is $P=10^{V-11}$, while the convectron gauge (which reads in a higher pressure regime, was scaled via the formula $P=10^{V-4}$.

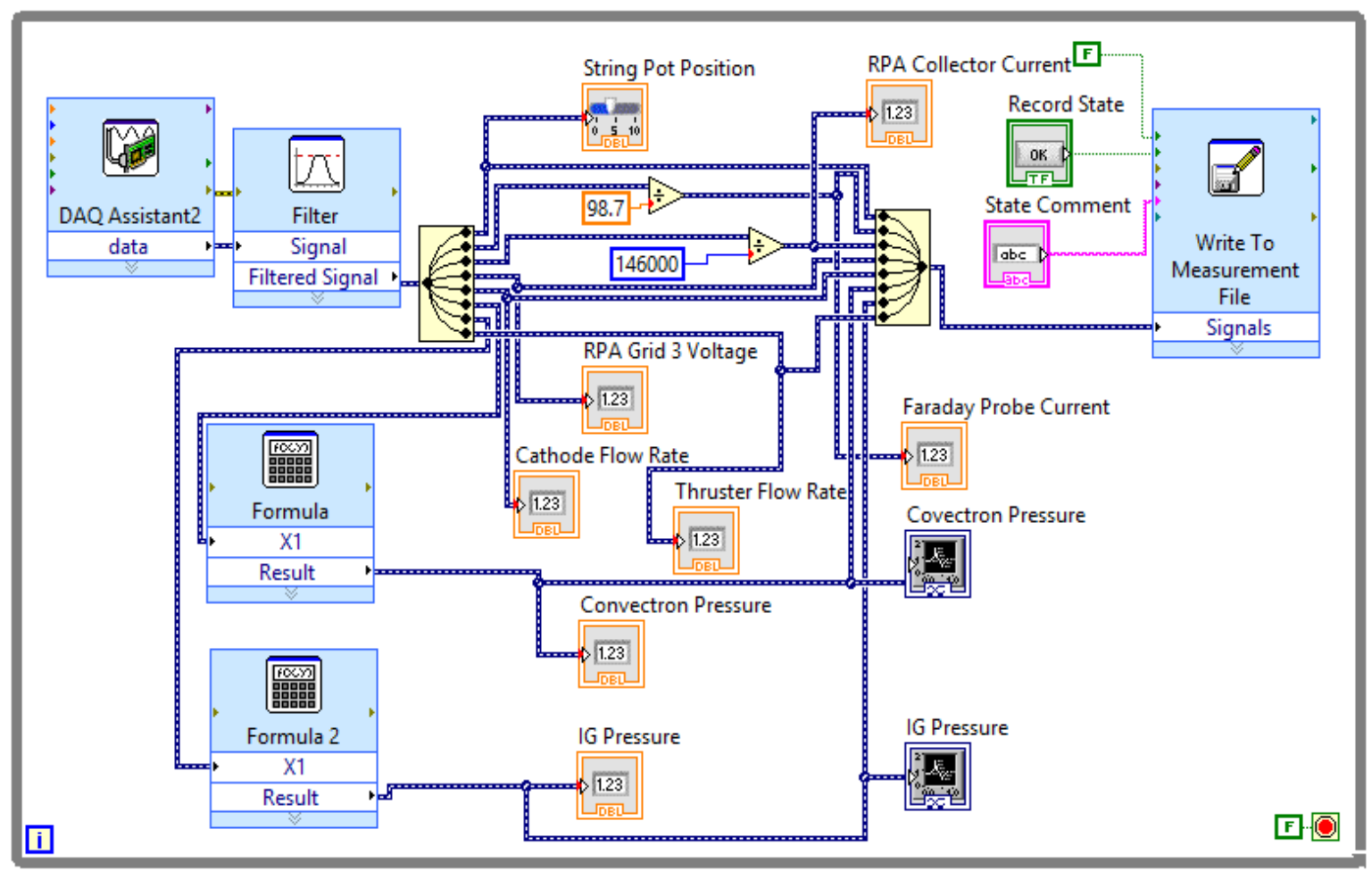

Figure 44. LabVIEW Main Loop. 


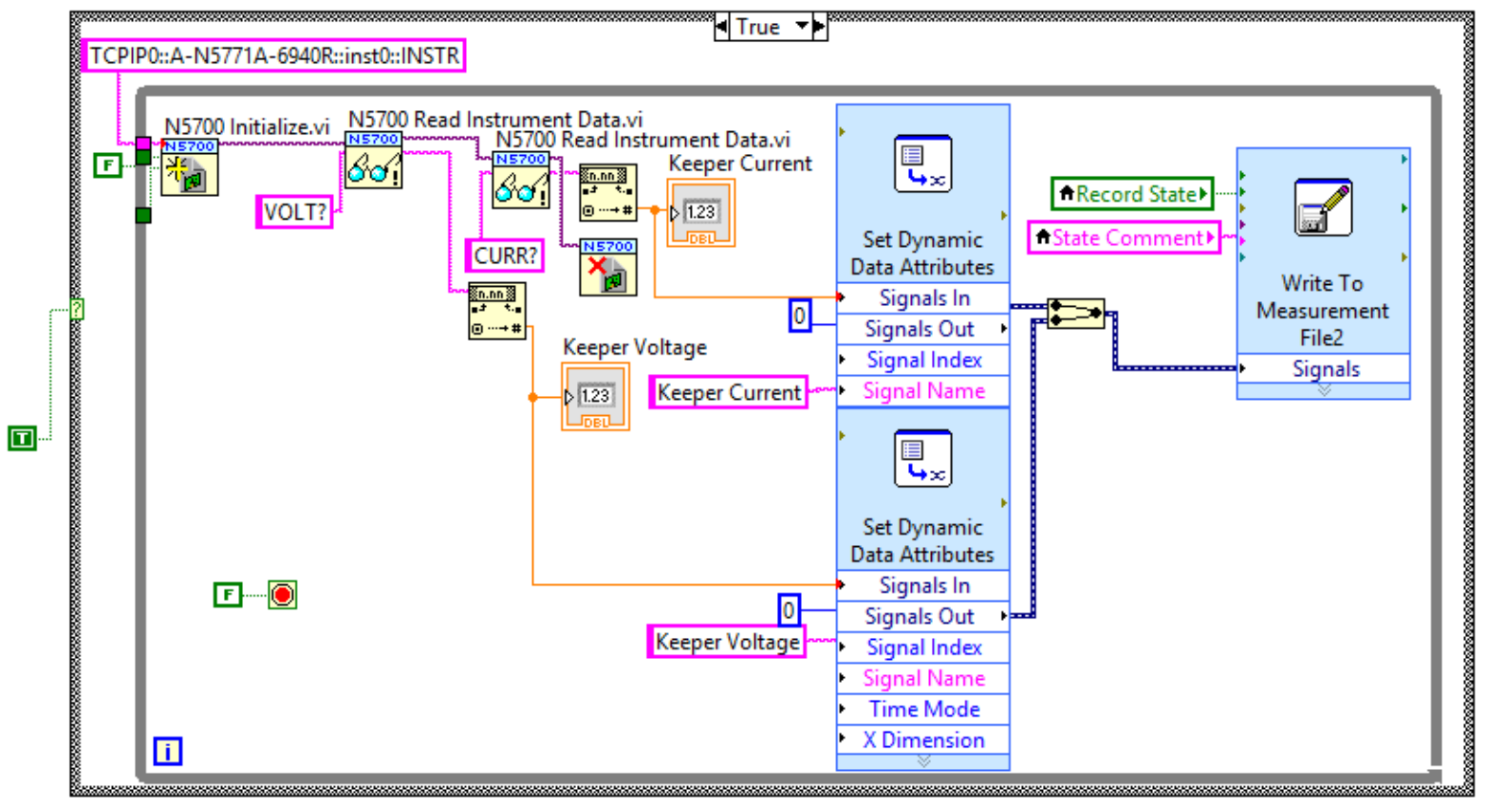

Figure 45. LabVIEW Keeper Loop. 